Pacific Northwest National Laboratory

Operated by Battelle for the U.S. Department of Energy

\title{
Hanford Contaminant Distribution Coefficient Database and Users Guide
}

\author{
K. J. Cantrell \\ R. J. Serne \\ G. V. Last
}

May 2002

Prepared for the U.S. Department of Energy under Contract DE-AC06-76RL01830 


\title{
DISCLAIMER
}

This report was prepared as an account of work sponsored by an agency of the United States Government. Neither the United States Government nor any agency thereof, nor Battelle Memorial Institute, nor any of their employees, makes any warranty, express or implied, or assumes any legal liability or responsibility for the accuracy, completeness, or usefulness of any information, apparatus, product, or process disclosed, or represents that its use would not infringe privately owned rights. Reference herein to any specific commercial product, process, or service by trade name, trademark, manufacturer, or otherwise does not necessarily constitute or imply its endorsement, recommendation, or favoring by the United States Government or any agency thereof, or Battelle Memorial Institute. The views and opinions of authors expressed herein do not necessarily state or reflect those of the United States Government or any agency thereof.

\author{
PACIFIC NORTHWEST NATIONAL LABORATORY \\ operated by \\ BATTELLE \\ for the \\ UNITED STATES DEPARTMENT OF ENERGY \\ under Contract DE-AC06-76RL01830
}

(3) This document was printed on recycled paper. 


\title{
Hanford Contaminant Distribution Coefficient Database and Users Guide
}

\author{
K. J. Cantrell \\ R. J. Serne \\ G. V. Last
}

May 2002

Prepared for

the U.S. Department of Energy

under Contract DE-AC06-76RL01830

Pacific Northwest National Laboratory

Richland, Washington 99352 


\section{Acknowledgments}

The authors wish to express their gratitude to Moses N. Jarayssi (Bechtel Hanford, Inc.), Manager of the Characterization of Systems Task of the Groundwater/Vadose Zone Integration Project, for his support of this work. The authors want to thank Wooyong Um and Wayne J. Martin for their technical and managerial reviews of this work, and Launa Morasch, Kathy Neiderhiser, and Lila Andor for their technical editing and text processing of this report. 


\section{Summary}

This document compiles in a single source the $\mathrm{K}_{\mathrm{d}}$ values measured with Hanford sediment for radionuclides and toxic compounds that have the greatest potential for driving risk to human health and safety in the vadose zone and groundwater at the Hanford Site. A small number of available $\mathrm{K}_{\mathrm{d}}$ values that had limited documentation and could not be readily evaluated were excluded from this compilation. In addition to the actual $\mathrm{K}_{\mathrm{d}}$ values, all significant experimental parameters and solution and sediment characterization data associated with these $K_{d}$ values have been compiled and documented as part of this task. These data will be compiled and made available on a sitewide database. This database will be periodically updated as new data become available.

In general, with proper data selection and application to appropriate conditions, the linear adsorption model $\left(\mathrm{K}_{\mathrm{d}}\right.$ model) approach is likely to be adequate for modeling transport through the Hanford system, especially for the far field where geochemical conditions remain fairly constant and contaminant loading of the adsorption sites is low. However, in some situations the linear adsorption model will not be appropriate, such as where large changes in chemical conditions occur (i.e., underneath a leaking highlevel waste tank). In such cases, rapidly changing chemical conditions will result in large changes in reactivity of the contaminants with surface sites and result in large changes in $\mathrm{K}_{\mathrm{d}}$ values. In other words, $\mathrm{K}_{\mathrm{d}}$ values are significantly dependent on multiple chemical parameters such as $\mathrm{pH}$, sodium ion concentration, or contaminant concentration. In addition, high concentrations of contaminants have the potential to saturate adsorptions sites. An inherent assumption of the linear adsorption model approach is that the adsorption sites never become saturated. These limitations of the linear adsorption model approach have been dramatically illustrated by the recent work conducted on $\mathrm{Cs}(\mathrm{I})$ adsorption on Hanford sediment under conditions expected underneath a leaking high-level waste tank.

When modeling adsorption from solutions whose compositions exceed the empirical range applicable for available $\mathrm{K}_{\mathrm{d}}$ measurements, additional measurements should be made to broaden the applicable range. In other cases, where the solution compositions and/or other adsorption parameters are not appropriate for the $\mathrm{K}_{\mathrm{d}}$ model approach, a more sophisticated (mechanistic) adsorption model should be considered.

In order to match $\mathrm{K}_{\mathrm{d}}$ values to a particular transport scenario, appropriate matching of solution chemistry with the chemical condition used in the $\mathrm{K}_{\mathrm{d}}$ determinations must be made. The differences in sediment mineralogy are generally of secondary importance to differences in solution chemistry with regard to $\mathrm{K}_{\mathrm{d}}$ values. Guidance on how to make corrections for gravel content is provided in this document. The importance of using a qualified geochemist for selecting appropriate $\mathrm{K}_{\mathrm{d}}$ is stressed. 


\section{Contents}

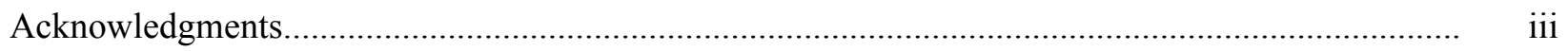

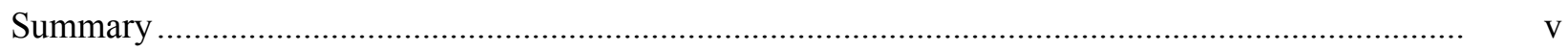

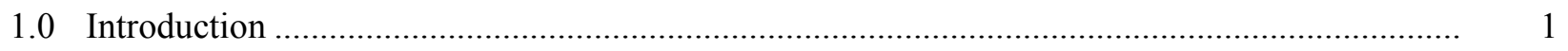

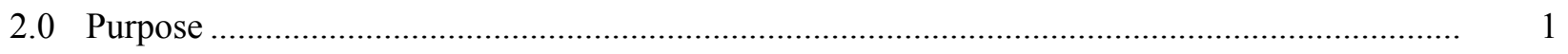



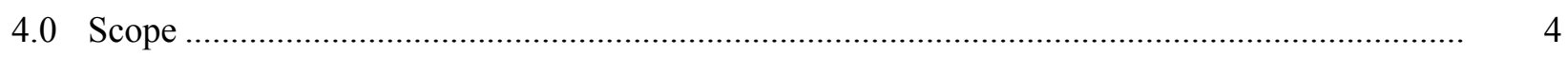

4.1 Distribution Coefficient and Background Characterization Data Catalog/Database ........... 5

4.2 Guidance for Selection of Distribution Coefficients ................................................. 5

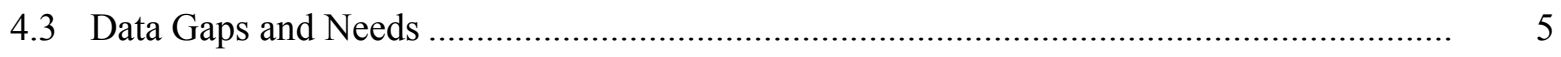

5.0 Catalog/Database of Distribution Coefficients ............................................................... 5

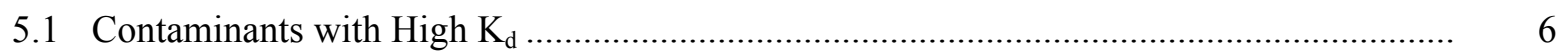

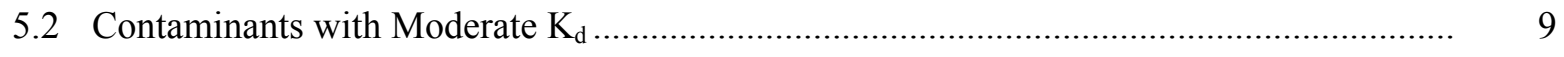

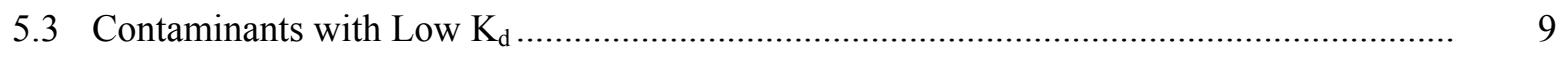

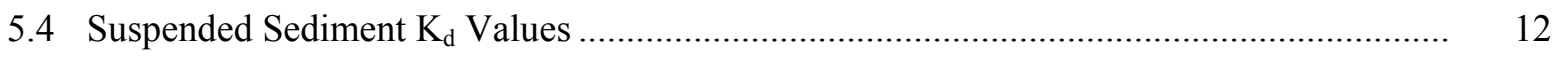

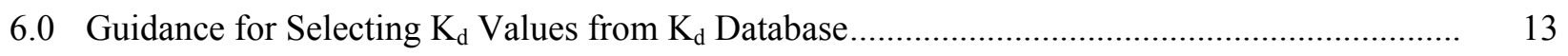

7.0 Data Gaps, Technical Needs, and Recommendations ...................................................... 14

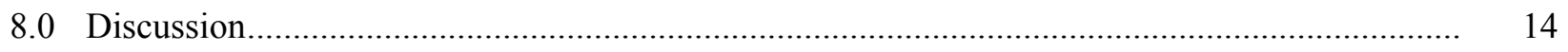

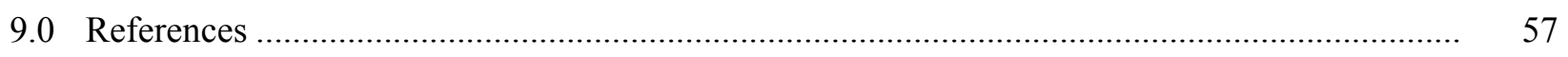

Appendix A - Aqueous Phase Characteristics ........................................................................ A.1

Appendix B - Solid Phase Characteristics ....................................................................... B. 1

Appendix C - Carbon Tetrachloride and Acetate $\mathrm{K}_{\mathrm{d}}$ Values Appropriate for Hanford Soils.............. C. C.1 


\section{Tables}

1 Range of $\mathrm{K}_{\mathrm{d}}$ Values Determined for the Immobilized Low-Activity Waste Performance



$2 \mathrm{R}_{\mathrm{f}}$ Values Calculated from the Range of $\mathrm{K}_{\mathrm{d}}$ Values for ${ }^{129} \mathrm{I},{ }^{237} \mathrm{~Np},{ }^{79} \mathrm{Se},{ }^{99} \mathrm{Tc}$, and ${ }^{234 / 235 / 238} \mathrm{U}$ Estimated for the Immobilized Low-Activity Waste Performance Assessment Far Field ........... $\quad 4$

3 Contaminant Distribution Coefficient Data for Americium ............................................... 15

4 Contaminant Distribution Coefficient Data for Cobalt.................................................... 18

5 Contaminant Distribution Coefficient Data for Cesium .................................................. 21

6 Contaminant Distribution Coefficient Data for Nickel....................................................... 26

7 Contaminant Distribution Coefficient Data for Lead .................................................... 28

8 Contaminant Distribution Coefficient Data for Plutonium................................................. 30

9 Contaminant Distribution Coefficient Data for Strontium ............................................... 33

10 Contaminant Distribution Coefficient Data for Chromium .................................................. 41

11 Contaminant Distribution Coefficient Data for Iodine .......................................................... 42

12 Contaminant Distribution Coefficient Data for Nitrate ......................................................

13 Contaminant Distribution Coefficient Data for Neptunium ................................................. 45

14 Contaminant Distribution Coefficient Data for Selenium ..................................................

15 Contaminant Distribution Coefficient Data for Technetium ............................................... 50

16 Contaminant Distribution Coefficient Data for Uranium ................................................. 53

17 Contaminant Distribution Coefficient Data for Carbon Tetrachloride .................................... 56 


\subsection{Introduction}

Adsorption is one of the primary mechanisms that control or retard the migration of many contaminants in the vadose zone and groundwater. The most common method used to describe contaminant adsorption on complicated matrices such as soil and sediment is the distribution coefficient or $\mathrm{K}_{\mathrm{d}}$ model:

$$
\mathrm{K}_{\mathrm{d}}=\mathrm{S} / \mathrm{C}_{\mathrm{aq}}
$$

where $\quad \mathrm{S}=$ the concentration of the contaminant on the solid

$\mathrm{C}_{\mathrm{aq}}=$ the concentration in the aqueous phase.

It is generally assumed that the $\mathrm{K}_{\mathrm{d}}$ is at equilibrium and reversible, although this is not always true. The primary drawback of this approach is that the model is empirical and should be applied only to conditions under which the $\mathrm{K}_{\mathrm{d}}$ was measured. This condition can be relaxed if a varying parameter is known to have no or minimal influence on adsorption of the contaminant of interest. The primary advantages of this approach are that it is simple and can be applied to complex matrices and solutions for which it would be difficult or impossible to obtain all the required mechanistic surface adsorption data.

An extensive review of adsorption models is beyond the scope of this report; for detailed reviews of adsorption modeling using empirical (including the $\mathrm{K}_{\mathrm{d}}$ model) and mechanistic approaches, see Lindsay (1979), Stumm and Morgan (1996), Langmuir (1997), and EPA (1999).

This work supports the Characterization of Systems Task of the Groundwater/Vadose Zone (GW/VZ) Integration Project. The Characterization of Systems Task is responsible for establishing a consistent set of data, parameters, and conceptual models to support efforts at the Hanford Site to estimate contaminant migration and impact (DeLamare 2000). As part of these efforts, the Characterization of Systems Task is assembling a series of catalogs and databases to identify the depth and breadth of existing data and to facilitate access to those data. It is envisioned that these catalogs and databases will continue to evolve as other existing data is found and new data collected.

This report joins four existing data catalogs that include the geologic data (Horton et al. 2001), vadose-zone hydraulic-property data (Freeman et al. 2001), surface and borehole geophysical data (Last and Horton 2000), and a catalog on release mechanisms (Riley and Lo Presti 2000). Efforts are being made to fully integrate these efforts and to facilitate the use of standardized nomenclature to which all stratigraphic, soil physics, and geochemistry data can be tied.

\subsection{Purpose}

The purpose of this work is to compile and thoroughly document all useful distribution coefficient data determined for Hanford sediment and related materials into one database. This report provides the 
background information to access the database and provides guidance on how to properly and effectively apply the information in the database to select appropriate $K_{d}$ values for numerical transport modeling efforts. A part of this work will be to identify significant data gaps and technical needs and make appropriate recommendations. The intent of this work is to maximize the value of existing $\mathrm{K}_{\mathrm{d}}$ data, ensure consistency, and eliminate duplication of effort among various programs.

An additional purpose of this work is to increase the defensibility of the linear isotherm model that will be used in SAC Rev. 1 and other up coming performance assessments. The work will provide transport modelers guidance for selecting appropriate $K_{d}$ values for various conditions that are consistent and scientifically defensible rather than excessively conservative.

\subsection{Technical Need}

Understanding the flux of contaminants through the vadose zone to the groundwater under varying geologic, hydrologic, and chemical conditions is key to making technically credible and sound decisions regarding soil site characterization and remediation, single-shell tank retrieval, and waste site closures (DOE 2000). One of the principal needs identified in the science and technology roadmap (DOE 2000) is the need to improve the conceptual and numerical models that describe the location of contaminants today, and to provide the basis to forecast future movement of contaminants on both site-specific and sitewide scales.

The state-of-knowledge (DOE 1999) and preliminary concepts ${ }^{1}$ documents describe the importance of geochemical processes on the transport of contaminants through the vadose zone. These processes have been identified in the international list of Features, Events, and Processes (FEPs) and included in the list of relevant Hanford Site FEPs (Soler et al. 2001). The current vision for the Hanford sitewide cumulative risk assessment as performed using the System Assessment Capability (SAC), Rev. 0 and Rev. 1, is to represent adsorption using the linear isotherm (empirical distribution coefficient, $\mathrm{K}_{\mathrm{d}}$ ) model.

Comments from the Integration Project Expert Panel indicate that work is required to adequately justify the applicability of the linear sorption model and to identify and defend the range of $\mathrm{K}_{\mathrm{d}}$ values that are adopted for assessments. The work plans developed for the science and technology efforts, SAC, and the core projects must answer directly the question of the scientific basis for the application of the linear sorption isotherm model to the complex waste on the Hanford Site.

The reason that well documented justification is required for using the linear sorption $\left(\mathrm{K}_{\mathrm{d}}\right)$ model is that this approach is strictly empirical and is often applicable only under a limited range of physicalchemical conditions. As a result, $\mathrm{K}_{\mathrm{d}}$ values can be applied with confidence only to conditions under

\footnotetext{
${ }^{1}$ Groundwater/Vadose Zone Integration Project, Preliminary System Assessment Capability Concepts for Architecture, Platform and Data Management, September 30, 1999, http://www.bhi-erc.com/vadose/ Workgrps/SAC/Report/9-30rep.pdf
} 
which the linear adsorption isotherm has been demonstrated to be applicable. If the sediment/soil mineralogy or physical properties, solution chemistry, or contaminant loading/concentration of the system to be modeled is significantly different than that for which the $\mathrm{K}_{\mathrm{d}}$ values were determined, significant error in the estimated transport rates could result. This is because many factors can affect the degree to which a particular contaminant adsorbs to a particular sediment or soil. These factors include sediment mineralogy and surface area, major ion concentration (complexation and competitive adsorption), $\mathrm{pH}$ of the solution, and the concentration of the adsorbate in solution and on the adsorbent. Also, the application of expert geochemical knowledge can often significantly reduce the number of variables that must be considered to evaluate $\mathrm{K}_{\mathrm{d}}$ values for each particular contaminant.

To illustrate how significant variation in $\mathrm{K}_{\mathrm{d}}$ values can affect transport model estimates, the range of $\mathrm{K}_{\mathrm{d}}$ values determined for the Immobilized Low-Activity Waste Performance Assessment (Kaplan and Serne 2000) will be used to calculate the variation in estimates of the retardation factor. In the first performance assessment, ${ }^{129} \mathrm{I},{ }^{237} \mathrm{~Np},{ }^{79} \mathrm{Se},{ }^{99} \mathrm{Tc}$, and ${ }^{234 / 235 / 238} \mathrm{U}$ were identified as radionuclides that posed the greatest potential health hazard. The estimated range of $\mathrm{K}_{\mathrm{d}}$ values determined for these contaminants are shown in Table 1.

The retardation factor is a measure of the ratio of the average linear velocity of water divided by the average linear velocity of the contaminant. The retardation factor can be calculated using the following equation:

$$
\mathrm{R}_{\mathrm{f}}=1+\left(\mathrm{K}_{\mathrm{d}} \rho_{\mathrm{b}}\right) / \theta
$$

where

$$
\begin{aligned}
\mathrm{R}_{\mathrm{f}} & =\text { the retardation factor (unitless) } \\
\rho_{\mathrm{b}}(\mathrm{kg} / \mathrm{L}) & =\text { the bulk density } \\
\theta(\mathrm{L} / \mathrm{L}) & =\text { the volumetric water content. }
\end{aligned}
$$

By assuming a bulk density of $1.86 \mathrm{~kg} / \mathrm{L}$ and a water content of $0.30 \mathrm{~L} / \mathrm{L}$, Equation 2 can be simplified to:

$$
\mathrm{R}_{\mathrm{f}}=1+6.2 \mathrm{~K}_{\mathrm{d}}
$$

The retardation factors calculated from the data in Table 1 are shown in Table 2. These results illustrate the high degree of uncertainty associated with model estimates of contaminant mobility at Hanford based on available distribution coefficients. For example, the range of retardation factors for ${ }^{129} \mathrm{I},{ }^{99} \mathrm{Tc}$, and ${ }^{234 / 235 / 238} \mathrm{U}$ indicate that the transport of these contaminants could vary from practically

Table 1. Range of $K_{d}$ Values Determined for the Immobilized Low-Activity Waste Performance Assessment Far Field for ${ }^{129} \mathrm{I},{ }^{237} \mathrm{~Np},{ }^{79} \mathrm{Se},{ }^{99} \mathrm{Tc}$, and ${ }^{234 / 235 / 238} \mathrm{U}$ (Kaplan and Serne 2000)

\begin{tabular}{|c|c|c|c|c||}
\hline \hline $\mathrm{K}_{\mathrm{d}}\left({ }^{129} \mathrm{I}\right)$ & $\mathrm{K}_{\mathrm{d}}\left({ }^{237} \mathrm{~Np}\right)$ & $\mathrm{K}_{\mathrm{d}}\left({ }^{79} \mathrm{Se}\right)$ & $\mathrm{K}_{\mathrm{d}}\left({ }^{99} \mathrm{Tc}\right)$ & $\mathrm{K}_{\mathrm{d}}\left({ }^{234 / 235 / 238} \mathrm{U}\right)$ \\
\hline \hline $0.0-15$ & $2-25$ & $3-15$ & $0.0-0.6$ & $0.1-80$ \\
\hline
\end{tabular}


Table 2. $\mathrm{R}_{\mathrm{f}}$ Values Calculated from the Range of $\mathrm{K}_{\mathrm{d}}$ Values for ${ }^{129} \mathrm{I},{ }^{237} \mathrm{~Np}$, ${ }^{79} \mathrm{Se},{ }^{99} \mathrm{Tc}$, and ${ }^{234 / 235 / 238} \mathrm{U}$ Estimated for the Immobilized Low-

Activity Waste Performance Assessment Far Field

\begin{tabular}{||c|c|c|c|c||}
\hline \hline $\mathrm{R}_{\mathrm{f}}\left({ }^{129} \mathrm{I}\right)$ & $\mathrm{R}_{\mathrm{f}}\left({ }^{237} \mathrm{~Np}\right)$ & $\mathrm{R}_{\mathrm{f}}\left({ }^{79} \mathrm{Se}\right)$ & $\mathrm{R}_{\mathrm{f}}\left({ }^{99} \mathrm{Tc}\right)$ & $\mathrm{R}_{\mathrm{f}}\left({ }^{234 / 235 / 238} \mathrm{U}\right)$ \\
\hline \hline $1-94$ & $13-156$ & $20-94$ & $1-4.7$ & $1.6-500$ \\
\hline
\end{tabular}

uninhibited to being significantly retarded (especially for ${ }^{99} \mathrm{Tc}$ and ${ }^{234 / 235 / 238} \mathrm{U}$ ). For ${ }^{237} \mathrm{~Np}$ and ${ }^{79} \mathrm{Se}$, the results indicate transport of these contaminants could range from moderately retarded to highly retarded.

As a result of this uncertainty, $\mathrm{K}_{\mathrm{d}}$ values selected for modeling purposes are typically the lowest value, or close to the lowest value, of the range of values. This conservative approach is prudent in the sense that it will tend to over estimate the transport of the contaminant rather than under estimate transport. By over estimating the transport of these contaminants, overly conservative remedial actions may be selected, leading to wasted efforts and unnecessary expenditures.

In order to reduce the uncertainty in the $\mathrm{K}_{\mathrm{d}}$ values for the contaminants of most importance, the variables that can significantly affect the $\mathrm{K}_{\mathrm{d}}$ values should be determined and thoroughly documented. These variables will be different for different contaminants. By documenting the variables that affect important contaminant $\mathrm{K}_{\mathrm{d}}$ values, and by combining this information with site-specific geochemical data, more precise and less conservative $\mathrm{K}_{\mathrm{d}}$ values can be selected. In addition, data gaps can be identified that will indicate where further experimental work should be conducted to reduce the uncertainty of the current $\mathrm{K}_{\mathrm{d}}$ value database.

\subsection{Scope}

The scope of this work is to provide an assessment and thorough documentation of the available contaminant distribution coefficients for Hanford sediment and related materials, and to document the conditions under which the $\mathrm{K}_{\mathrm{d}}$ values were determined. This work will provide a mechanism to select a range of $\mathrm{K}_{\mathrm{d}}$ values based on waste chemistry type, water geochemistry, mineralogy and, if possible, hydraulic properties that will minimize the range of uncertainty.

The scope of this work is focused on three main objectives:

- compilation/database of distribution coefficients and background characterization data for Hanford sediment with bibliography

- preparation of limited guidance for selecting distribution coefficients from the Hanford database

- identification of data gaps and needs. 


\subsection{Distribution Coefficient and Background Characterization Data Catalog/Database}

The first effort was aimed at producing a catalog of all useful contaminant distribution coefficient data applicable to Hanford sediment and related materials. These data were evaluated and rated in terms of quality, based on how well the values are documented. Documentation included availability of the original reference, availability of sediment characterization data, solution phase characterization data, contaminant concentrations in the solution phase and solid phase, and experimental procedures used. Some older data that does not contain adequate documentation regarding the solution or sediment characteristics has been intentionally excluded from this compilation. This document also provides a comprehensive bibliography of all available published documents that contain useful distribution coefficient data applicable to the Hanford Site. The distribution coefficient and background characterization data compiled in this data catalog will be entered into an electronic database for future management and configuration control.

\subsection{Guidance for Selection of Distribution Coefficients}

Limited guidance to assist in the selection of distribution coefficients was developed for modelers who are not expert geochemists so that they will be able to use this information more effectively. It is highly recommended that a knowledgeable geochemist with firsthand experience in the area of contaminant adsorption, speciation chemistry, and Hanford $\mathrm{K}_{\mathrm{d}}$ values be consulted prior to final selection of $\mathrm{K}_{\mathrm{d}}$ values for conducting modeling efforts with critical outcomes.

\subsection{Data Gaps and Needs}

A logical outcome from the preparation of this document will be to identify data gaps and technical needs, and to make recommendations for resolving these needs. This will include identification of further experimental work that should be conducted to maximize the effectiveness of future transport modeling efforts.

\subsection{Catalog/Database of Distribution Coefficients}

This document presents distribution coefficient and background characterization data in a series of tables and appendices that are believed to represent $90 \%$ of the existing data available for the Hanford Site. The compilation of new and existing data is expected to continue and will result in updated versions of this data catalog/database. 
Compilations of distribution coefficients for $\mathrm{Am}(\mathrm{III}), \mathrm{Co}(\mathrm{II}), \mathrm{Cr}(\mathrm{VI}), \mathrm{Cs}(\mathrm{I}), \mathrm{I}(\mathrm{I}), \mathrm{Ni}(\mathrm{II}), \mathrm{NO}_{3}{ }^{-}, \mathrm{Np}(\mathrm{V})$, $\mathrm{Pb}(\mathrm{II}), \mathrm{Pu}$ (valence unspecified), $\mathrm{Se}(\mathrm{VI}), \mathrm{Sr}(\mathrm{II}), \mathrm{Tc}(\mathrm{VII}), \mathrm{U}(\mathrm{VI})$, and $\mathrm{CCl}_{4}$ are presented in Tables 3 through 17 following the main text of this report. Where applicable and available, the following information was included in the tables along with the $\mathrm{K}_{\mathrm{d}}$ values:

- name used to identify the aqueous phase

- name used to identify the solid phase

- experimental method

- standard deviation of the $\mathrm{K}_{\mathrm{d}}$ values (if applicable and available)

- equilibration time

- initial concentration of the adsorbate in the solution

- solution/solid ratio used in the experiment

- source reference.

In addition to these data, more detailed information regarding the composition of the solutions (aqueous phase) is given in Appendix A. More detailed information regarding the sediment composition and characteristics (solid phase) is given in Appendix B.

For the purposes of discussion, these contaminants have been grouped into three classifications:

1. contaminants with very high $\mathrm{K}_{\mathrm{d}}$ values with very little potential for migration through the vadose zone or groundwater except under extreme conditions [Am(III), $\mathrm{Co}(\mathrm{II}), \mathrm{Cs}(\mathrm{I}), \mathrm{Ni}(\mathrm{II}), \mathrm{Pb}(\mathrm{II})$, and $\mathrm{Pu}$ ]

2. contaminants with moderate $\mathrm{K}_{\mathrm{d}}$ values with moderate potential for migration through the vadose zone or groundwater [Sr(II)]

3. contaminants with low $\mathrm{K}_{\mathrm{d}}$ values with relatively high potential for migration through the vadose zone or groundwater [Cr(VI), I(I), $\mathrm{NO}_{3}{ }^{-}, \mathrm{Np}(\mathrm{V}), \mathrm{Se}(\mathrm{VI}), \mathrm{Tc}(\mathrm{VII}), \mathrm{U}(\mathrm{VI})$, and $\left.\mathrm{CCl}_{4}\right]$.

Unless otherwise indicated, all $\mathrm{K}_{\mathrm{d}}$ values were measured on sediment that was sieved to $<2 \mathrm{~mm}$ (i.e., sum of sand-, silt-, and clay-size fractions). Appropriate corrections should be made when applying these values to actual field conditions with significant amounts of material $>2 \mathrm{~mm}$ (i.e., gravel) (Kaplan et al. 2000). Note, that in some cases negative $K_{d}$ values are reported. Negative $K_{d}$ values are physically impossible and are the result of experimental artifacts. For modeling purposes, these values should be taken to be equal to $0.0 \mathrm{~mL} / \mathrm{g}$. The negative values are included in the table to provide a sense of the magnitude of experimental error that can occur in these numbers.

\subsection{Contaminants with High $\mathbf{K}_{\mathrm{d}}$}

Americium. $\mathrm{K}_{\mathrm{d}}$ values for Am(III) and associated information are compiled in Table 3. Routson et al. $(1975,1976)$ measured Am(III) $K_{d}$ values under conditions that closely approximate those of natural Hanford groundwater. These results indicate that $\mathrm{Am}(\mathrm{III}) \mathrm{K}_{\mathrm{d}}$ values are high under natural conditions. Delegard and Barney (1983) measured Am(III) $\mathrm{K}_{\mathrm{d}}$ values under highly basic pH conditions (1 M and $4 \mathrm{M}$ 
$\mathrm{NaOH}$ ) with various concentrations of chelating agents. The results of this work indicate that Am(III) $\mathrm{K}_{\mathrm{d}}$ values under highly basic conditions in the absence of ethylenediaminetetraacetic acid are also high. At these high $\mathrm{pH}$ values, it is not clear if the high Am(III) $\mathrm{K}_{\mathrm{d}}$ values measured are the result of adsorption or a combination of adsorption and precipitation; it is certain that Am(III) is highly immobile under these conditions. When high concentrations of ethylenediaminetetraacetic acid are present $(0.05$ to $0.15 \mathrm{M})$ Am(III) $\mathrm{K}_{d}$ values are significantly reduced to low or moderate values. Am(III) $\mathrm{K}_{d}$ values measured under acidic conditions $(\mathrm{pH}<3)$ are low $(<1 \mathrm{~mL} / \mathrm{g})$.

The general conclusions that can be drawn from these results are 1) Am(III) is generally immobile under typical groundwater $\mathrm{pH}$ values and above, 2) high concentrations of ethylenediaminetetraacetic acid significantly increase the mobility of Am(III), and 3) Am(III) is very mobile at pH values below 3.

Cobalt. Table 4 contains the available $\mathrm{Co}(\mathrm{II}) \mathrm{K}_{\mathrm{d}}$ values applicable to Hanford conditions. Serne et al. (1993) and Gee and Campbell (1980) determined $\mathrm{K}_{\mathrm{d}}$ values for Co(II) under natural Hanford groundwater conditions. These results indicate that under these conditions $\mathrm{Co}(\mathrm{II})$ adsorption is high. $\mathrm{K}_{\mathrm{d}}$ values for Co(II) also were determined by Delegard and Barney (1983) under highly basic conditions $(1 \mathrm{M}$ and $4 \mathrm{M} \mathrm{NaOH})$ and in the presence of various concentrations of chelating agents. These results indicate that a highly basic condition greatly reduces $\mathrm{Co}$ (II) (at trace concentrations) adsorption onto Hanford sediment. This is due to the formation of negatively charged hydrolysis species under strongly basic conditions. The combination of highly basic conditions and high concentrations of ethylenediaminetetraacetic acid reduces Co(II) adsorption to practically nil. Cantrell and Serne (1993) measured $\mathrm{K}_{\mathrm{d}}$ values for Co(II) under natural Hanford groundwater conditions and with Hanford groundwater spiked with ethylenediaminetetraacetic acid and $\mathrm{CN}^{-}$. It was determined that addition of 25 ppb ethylenediaminetetraacetic acid reduced $\mathrm{K}_{d}$ values for $\mathrm{Co}(\mathrm{II})$ from 2,500 to $1,070 \mathrm{~mL} / \mathrm{g}$. Further reductions in $\mathrm{K}_{\mathrm{d}}$ values occurred as the ethylenediaminetetraacetic acid concentration was increased. At $200 \mathrm{ppb}$ ethylenediaminetetraacetic acid, the average $K_{d}$ was determined to be $71 \mathrm{~mL} / \mathrm{g}$ and at 2,000 ppb, the average $K_{d}$ was $3.3 \mathrm{~mL} / \mathrm{g}$. $\mathrm{CN}^{-}$was found to reduce $\mathrm{Co}(\mathrm{II})$ adsorption even more dramatically than ethylenediaminetetraacetic acid. At $150 \mathrm{ppb} \mathrm{CN}^{-}$, the average $\mathrm{Co}$ (II) $\mathrm{K}_{\mathrm{d}}$ value was determined to be $1.9 \mathrm{~mL} / \mathrm{g}$ and at 2,000 $\mathrm{ppb} \mathrm{CN}^{-} \mathrm{Co}$ (II) $\mathrm{K}_{\mathrm{d}}$ value was reduced even further to $0.0 \mathrm{~mL} / \mathrm{g}$.

The general conclusions that can be drawn from these results are 1) Co(II) is highly immobile under normal Hanford groundwater conditions, 2) highly basic conditions dramatically reduce Co(II) adsorption, and 3) moderate to high concentrations of $\mathrm{CN}^{-}$and high ethylenediaminetetraacetic acid concentrations greatly reduces $\mathrm{Co}(\mathrm{II})$ adsorption.

Cesium. A large body of data for Cs(I) adsorption onto Hanford sediment exists. The most useful $\mathrm{K}_{\mathrm{d}}$ values have been compiled in Table 5. Under normal Hanford conditions, Cs(I) adsorption is high with $\mathrm{K}_{\mathrm{d}}$ values in excess of $1,000 \mathrm{~mL} / \mathrm{g}$. Even in the presence of acidic process waste, Cs(I) adsorption remains high. This is partially due to the high acid neutralizing capacity of Hanford sediment resulting from its generally high carbonate content. The $\mathrm{pH}$ values measured for acidic process waste (initially $\mathrm{pH} 3.5$ ) after contact with Hanford sediment was 4.1 to 7.5 (at solution to solid ratios of 30). Gee and Campbell (1980) demonstrated that high concentrations of $\mathrm{K}^{+}$can dramatically reduce $\mathrm{Cs}(\mathrm{I})$ adsorption; however, such high $\mathrm{K}^{+}$concentrations are not likely to occur at the Hanford Site. Serne et al. (1998) has shown that various simulated tank (T-106) waste ( $\mathrm{pH} 12$, with various salts at high concentration) can 
significantly reduce $\mathrm{Cs}(\mathrm{I})$ adsorption. The most dramatic decrease in $\mathrm{Cs}(\mathrm{I})$ adsorption occurs when high $\mathrm{Ca}\left(\mathrm{NO}_{3}\right)_{2}(3.5 \mathrm{M})$ is included as a component of the simulated tank waste (along with relatively high concentrations of $\mathrm{NH}_{4}{ }^{+}$and $\mathrm{K}^{+}$). REDOX liquors that have much higher base (pH>14), $\mathrm{Al}, \mathrm{Na}$, and nitrate concentrations, have been found to have higher $\mathrm{K}_{d}$ values than those of the T-106 tank waste simulants. It has been hypothesized that precipitation of high-surface-area aluminum-hydroxide phases may be responsible for this effect Serne et al. (1998). It is also likely that the much lower concentrations of $\mathrm{Ca}^{2+}, \mathrm{NH}_{4}{ }^{+}$, and $\mathrm{K}^{+}$in the REDOX liquors were also very important factors.

Zachara et al. (2002) have presented a detailed mass action ion exchange model for Cs(I) adsorption onto Hanford sediment. This model is sensitive to the concentration of Cs(I) in the system because of selective adsorption sites (frayed edge sites on mica minerals) that are present in low concentrations that control Cs(I) adsorption at low aqueous $\mathrm{Cs}(\mathrm{I})$ concentrations. In addition, high salt concentrations that exist in tank waste greatly reduces $\mathrm{Cs}(\mathrm{I})$ adsorption. As a result of this work, it is clear that modeling $\mathrm{Cs}(\mathrm{I})$ adsorption in the vicinity of a tank leak will not be amenable to modeling with a single linear adsorption isotherm.

In summary, it appears that Cs(I) transport through the Hanford Site vadose zone and groundwater will be negligible except under conditions of extremely high salt concentration $\left[\mathrm{Ca}^{2+}, \mathrm{NH}_{4}^{+}\right.$, and $\mathrm{K}^{+}$are particularly good competitors for adsorption sites with Cs(I)].

Nickel. $\mathrm{Ni}(\mathrm{II}) \mathrm{K}_{\mathrm{d}}$ values compiled for this database are shown in Table 6. Adsorption of Ni(II) onto Hanford sediment is high but somewhat less than that of $\mathrm{Co}(\mathrm{II})$. Ni(II) adsorption data are available as a function of contact time and initial concentration of $\mathrm{Ni}(\mathrm{II}) . \mathrm{K}_{\mathrm{d}}$ values for the radionuclides previously discussed were all conducted at trace concentrations. Increasing the equilibration time results in significant increases in the measured $\mathrm{K}_{\mathrm{d}}$ values. In addition, increasing the concentration of $\mathrm{Ni}$ (II) initially added to solution significantly reduces the measured $\mathrm{K}_{\mathrm{d}}$ values. The highest $\mathrm{Ni}$ (II) concentrations used in these experiments are near the solubility limits expected in Hanford groundwater (Rhodes et al. 1994).

It is apparent, from the available results for $\mathrm{Ni}(\mathrm{II})$ adsorption onto Hanford sediment under normal groundwater conditions, that the mobility of nickel is limited. Even at the highest nickel concentrations studied $\left(5 \times 10^{-5} \mathrm{M}\right)$, a retardation factor in excess of 300 can be expected. As the Ni(II) concentrations decrease and the contact time increases, retardation factors in excess of 4,000 are expected.

Lead. $\mathrm{Pb}(\mathrm{II}) \mathrm{K}_{\mathrm{d}}$ values determined with Hanford sediment are presented in Table 7. $\mathrm{Pb}$ (II) adsorption onto Hanford sediment also is high, even higher than that of $\mathrm{Co}(\mathrm{II})$. As is the case for $\mathrm{Ni}(\mathrm{II}), \mathrm{K}_{\mathrm{d}}$ values for $\mathrm{Pb}$ (II) are available as a function of time and initial concentration of $\mathrm{Ni}(\mathrm{II})$. In a similar fashion to that observed for $\mathrm{Ni}(\mathrm{II}), \mathrm{K}_{\mathrm{d}}$ values for $\mathrm{Pb}(\mathrm{II})$ are observed to increase with increasing equilibration times and decreasing initial $\mathrm{Pb}(\mathrm{II})$ solution concentrations. Because the solubility limit for $\mathrm{Pb}(\mathrm{II})$ in Hanford Site groundwater is significantly less than for Ni(II) (Rhoads et al. 1992), the maximum possible solution concentration for $\mathrm{Pb}$ (II) is much lower than that of $\mathrm{Ni}(\mathrm{II})$, as a result, the degree of site loading is significantly lower for $\mathrm{Pb}$ (II) than for $\mathrm{Ni}(\mathrm{II})$. These results show that under normal conditions $\mathrm{Pb}$ (II) is extremely immobile in the Hanford vadose zone and groundwater. 
Plutonium. Available $\mathrm{K}_{\mathrm{d}}$ values for $\mathrm{Pu}$ were measured on Hanford sediment have been compiled in Table 8 . The quantity and quality of $\mathrm{Pu}$ adsorption studies conducted with Hanford sediment are much less than those available for many other contaminants of interest at the Hanford Site. Delegard and Barney (1983) conducted a series of Pu adsorption experiments on Hanford sediment at high base concentrations and variable concentrations of chelating agents. From their results, it was demonstrated that even at high base concentrations Pu adsorption was moderately high. Combination of high base concentration and high ethylenediaminetetraacetic acid concentration reduced $\mathrm{Pu}$ adsorption the most; however, even under these conditions significant adsorption occurred. Hajek and Knoll (1966) conducted $\mathrm{Pu}$ adsorption experiments on Hanford sediment from high salt acid waste. Under these conditions, the $\mathrm{K}_{\mathrm{d}}$ values for Pu were determined to be less than 1. In another study conducted by Rhodes $(1952,1957)$, $\mathrm{K}_{\mathrm{d}}$ values for $\mathrm{Pu}$ were measured on Hanford sediment at different solution to solid ratios, variable initial $\mathrm{Pu}$ concentrations and a range of $\mathrm{pH}$ values from 0.5 to 14 . In general, these results indicate high $\mathrm{Pu}$ adsorption, except at very low $\mathrm{pH}$. The results of Rhodes at low and high $\mathrm{pH}$ are not consistent with the previous results discussed. It is possible that the high $\mathrm{K}_{\mathrm{d}}$ values determined by Rhodes resulted from precipitation as a result of the high initial $\mathrm{Pu}$ [stated to be $\mathrm{Pu}(\mathrm{IV})$ ] concentrations used in the experiments.

Based on the limited data available for $\mathrm{Pu}$, it appears that $\mathrm{Pu}$ will be fairly immobile except at very low $\mathrm{pH}$ values or high ethylenediaminetetraacetic acid concentrations.

\subsection{Contaminants with Moderate $K_{d}$}

Strontium. As was the case for Cs(I), a large number of studies of $\mathrm{Sr}$ (II) adsorption on Hanford sediment have been conducted. The $\mathrm{K}_{\mathrm{d}}$ values determined in these studies are listed in Table 9. Under most natural conditions, $\mathrm{Sr}(\mathrm{II})$ adsorption onto Hanford sediment is moderate with $\mathrm{K}_{\mathrm{d}}$ values that range from approximately 10 to 20 , although much higher values have been measured in fine-grained material, presumably due to the much higher clay content in these materials (Serne and LeGore 1996). Acidic conditions and high salt concentrations (calcium, magnesium, ammonium, and potassium in particular) can significantly reduce Sr(II) adsorption onto Hanford sediment. High concentrations of ethylenediaminetetraacetic acid can reduce $\mathrm{Sr}(\mathrm{II})$ adsorption to essentially zero. High $\mathrm{pH}$ conditions in the absence of high concentrations of competitive cations and ethylenediaminetetraacetic acid increases $\operatorname{Sr}(\mathrm{II})$ adsorption. In addition to greater adsorption, the higher $\mathrm{K}_{\mathrm{d}}$ values determined for $\mathrm{Sr}(\mathrm{II})$ at high $\mathrm{pH}$ may reflect some co-precipitation of $\mathrm{Sr}$ (II) into calcium/magnesium carbonates that could precipitate from groundwater as the $\mathrm{pH}$ is increased. It appears that under most circumstances strontium migration in Hanford sediment will be fairly limited.

\subsection{Contaminants with Low $K_{d}$}

Chromium. $\mathrm{Cr}(\mathrm{VI}) \mathrm{K}_{\mathrm{d}}$ values determined with Hanford sediment are presented in Table 10. Only a few studies of $\mathrm{Cr}(\mathrm{VI})$ adsorption on Hanford soil are available. These results indicate that under normal Hanford Site groundwater conditions, $\mathrm{Cr}(\mathrm{VI})$ adsorption is very low. Measured $\mathrm{K}_{\mathrm{d}}$ values range from 0 to 1 , with typical values being zero or close to zero. Under acidic conditions, adsorption of this anionic species becomes significant. This increase in adsorption with decreasing $\mathrm{pH}$ is expected for anions due to the increase in the number of positive surface sites on the sediment with decreasing $\mathrm{pH}$. An interesting observation with regard to $\mathrm{Cr}(\mathrm{VI})$ adsorption to Hanford sediment under acidic conditions, is that $\mathrm{K}_{\mathrm{d}}$ 
values show a significant increase with increasing equilibration time. It is speculated that this may be caused by slow release of ferrous iron from basalt in the Hanford sediment. The ferrous iron leached from the sediment can act as a reductant for $\mathrm{Cr}(\mathrm{VI})$. Reduction of $\mathrm{Cr}(\mathrm{VI})$ to $\mathrm{Cr}^{3+}$ would result in precipitation of $\mathrm{Cr}(\mathrm{OH})_{3}(\mathrm{~s})$ and result in the apparently high $\mathrm{K}_{\mathrm{d}}$ values. Except under acidic conditions adsorption of $\mathrm{Cr}(\mathrm{VI})$ is low to non-existent.

Iodine. Table 11 is a tabulation of $\mathrm{K}_{\mathrm{d}}$ values determined for $\mathrm{I}(\mathrm{I})$ adsorption onto Hanford sediment. Iodide $[\mathrm{I}(\mathrm{I})]$ is expected to be the dominant species of iodine in Hanford groundwater (Kaplan and Serne 2000). I(I) adsorption experiments generally indicate relatively low adsorption. Under typical Hanford Site groundwater conditions, $K_{d}$ values range from approximately 0 to $2 \mathrm{~mL} / \mathrm{g}$ with a range of 0 to $0.2 \mathrm{~mL} / \mathrm{g}$ being most typical. Consistent with anionic adsorption in general, acidic conditions appear to increase I(I) adsorption; however, sufficient data are not available to make a firm conclusion. Note that in most Hanford geochemical environments the $\mathrm{pH}$ does not drop below 7 because of the ubiquitous occurrence of carbonates in Hanford sediment and the preponderance of alkaline waste discharges. Also, long equilibration periods ( $>100$ days) result in a non-linear increase in $\mathrm{K}_{\mathrm{d}}$ values with time. The reason for this is unclear, but it is speculated that microbial activity may have played a significant role in this phenomenon. The formation of microbial colonies has been observed in a number of similar long-term adsorption experiments. These conditions are not expected to be typical of Hanford Site groundwater conditions, and it is recommended that these high values be ignored for typical transport modeling exercises.

Nitrate. The limited number of $\mathrm{K}_{\mathrm{d}}$ values determined for $\mathrm{NO}_{3}{ }^{-}$using Hanford sediment are shown in Table 12. Only one study is available in which nitrate adsorption was measured. These results indicate that nitrate adsorption is essentially zero (within experimental error).

Neptunium. $K_{d}$ values for $\mathrm{Np}(\mathrm{V})$ determined using Hanford sediment are compiled in Table 13. These data indicate $\mathrm{Np}(\mathrm{V})$ adsorption is generally moderate, with $\mathrm{K}_{\mathrm{d}}$ values in the general range of 2 to $30 \mathrm{~mL} / \mathrm{g}$. Lower values can result at contact times of 1 day or less and high calcium or ethylenediaminetetraacetic acid concentrations in solution. High solution $\mathrm{pH}$ values can result in much higher $\mathrm{K}_{\mathrm{d}}$ values. These results indicate that $\mathrm{Np}(\mathrm{V})$ from a tank leak should be minimal unless high ethylenediaminetetraacetic acid concentrations occur. Moderate migration of $\mathrm{Np}(\mathrm{V})$ could occur in the vadose zone and groundwater under natural Hanford conditions.

Selenium. A fair number of $\mathrm{Se}(\mathrm{VI}) \mathrm{K}_{\mathrm{d}}$ values have been determined using natural Hanford sediment and are listed in Table 14. These results indicate that at trace concentrations, adsorption of $\mathrm{Se}(\mathrm{VI})$ to Hanford sediment is low to moderate with $\mathrm{K}_{\mathrm{d}}$ values ranging from 3 to $10 \mathrm{~mL} / \mathrm{g}$. At higher $\mathrm{Se}(\mathrm{VI})$ concentrations, the $\mathrm{K}_{\mathrm{d}}$ values are lower $(0$ to $3 \mathrm{~mL} / \mathrm{g})$. Acidic conditions increase $\mathrm{Se}(\mathrm{VI})$ adsorption, and basic conditions reduce adsorption. This is consistent with the anionic character of $\mathrm{Se}(\mathrm{VI})$.

Technetium. Tc(VII) $\mathrm{K}_{\mathrm{d}}$ values measured on Hanford sediment are shown in Table 15. Tc(VII) adsorption is low under nearly all conditions relevant to the Hanford vadose zone and upper unconfined aquifer, with $\mathrm{K}_{\mathrm{d}}$ values ranging from zero to a high of approximately $1 \mathrm{~mL} / \mathrm{g}$; however, these high values also have high standard deviations. In one study, $\mathrm{K}_{\mathrm{d}}$ values approaching $1 \mathrm{~mL} / \mathrm{g}$ appeared to be most 
representative; however, this was not substantiated by any other studies. Under normal Hanford conditions, zero appears to be the most appropriate value, with a best estimate for the range as 0.0 to $0.1 \mathrm{~mL} / \mathrm{g}$.

Uranium. Table 16 contains $\mathrm{K}_{\mathrm{d}}$ values determined for $\mathrm{U}(\mathrm{VI})$ adsorption on Hanford sediment. Under natural Hanford groundwater conditions, $\mathrm{U}(\mathrm{VI})$ adsorption is moderate with $\mathrm{K}_{\mathrm{d}}$ values ranging from approximately 0.2 to 4 . Much higher $K_{d}$ values have been reported (Serne et al. 1993) but these values appear to be suspect. In the case of the TBS-1 results determined in Hanford groundwater, nothing is unusual about the sediment or groundwater. In the cases in which U(VI) adsorption was measured from acid process waste, adsorption should actually be lower as a result of the lower equilibrium $\mathrm{pH}$ values reached in these experiments. However, the reported adsorption from the acid process waste is much higher than observed in any other studies. For example, the maximum U(VI) adsorption onto natural sediment from natural groundwater indicate that an adsorption maximum occurs in the $\mathrm{pH}$ range of approximately 6 to 8, with lower adsorption occurring a lower $\mathrm{pH}$ due to protonation of the adsorption sites and a shift to more positively charged uranyl species in solution (Payne and White 1991; Davis 2001). Lower adsorption also occurs at higher $\mathrm{pH}$ values due to formation of more negatively charged uranyl-carbonate species and deprotonation of the surface sites. These previously reported high $\mathrm{K}_{\mathrm{d}}$ values for uranium have had a significant effect on recommended $\mathrm{K}_{\mathrm{d}}$ values. For example, the recommended range suggested in the Immobilized Low-Activity Waste Performance Assessment Far Field for ${ }^{234 / 235 / 238} \mathrm{U}$ is 0.1 to 80 (Kaplan and Serne 2000). Based on this analysis, a range of 0.2 to 4 is more likely to be appropriate. Uranium migration under natural Hanford conditions will be high to moderate with greater migration occurring at high and low $\mathrm{pH}$ values.

Carbon Tetrachloride. The only $\mathrm{K}_{\mathrm{d}}$ values available for Hanford sediment were measured by the author (unpublished data). The sediment used for these measurements were collected on November 5, 1991, from well 299-W11-27 at a depth of 200 feet. The measurements were made by adding $40 \mathrm{mls}$ of Hanford groundwater (well 6-S3-25) spiked with carbon tetrachloride at concentrations ranging from 5 to $10,000 \mathrm{ppb}$ to 1 gram of sediment $(<2 \mathrm{~mm})$ in glass vials with teflon sealing caps and equilibrating for several days. The mean and standard deviation for the measured $\mathrm{K}_{d}$ values was $-0.8 \pm 0.8 \mathrm{~mL} / \mathrm{g}$. The reason for the negative values is an artifact of the $K_{d}$ determination method (Relyea et al. 1980), in which equilibrium concentration values are subtracted from blank concentrations. Because carbon tetrachloride is so volatile any small losses from the blanks that are higher than the sample values will result in a negative $K_{d}$ value. As a result, it is assumed that the actual $K_{d}$ value for Hanford sediment is $0.0 \pm 0.8 \mathrm{~mL} / \mathrm{g}$.

Others (Truex et al. 2001) have used estimation methods based on the fraction of organic carbon in the soil and the water solubility of the compound of interest to estimate $K_{d}$ values for organic contaminants for Hanford sediment. However, these estimation methods are known to underestimate $\mathrm{K}_{\mathrm{d}}$ values for organic carbon concentrations below approximately $0.1 \%$. The average organic content for Hanford sediment is typically $0.03 \%$. As a result, these estimation methods are not generally useful for Hanford sediment.

A more reliable method to estimate carbon tetrachloride $K_{d}$ values for Hanford sediment is to compare with values measured on sediment that has low organic contents similar to that of Hanford 
sediment. This is reasonable because mineralogical differences do not have a significant impact on the adsorption of hydrophobic contaminants. Particle size range (surface area) will have a much more significant impact than mineralogy.

Brusseau et al. (1991) measured retardation factors for carbon tetrachloride in an aquifer material that was greater than $98 \%$ sand (Rabis baek). The fraction of organic carbon for this material was 0.00007 . Using the given bulk density and pore water content, a $\mathrm{K}_{\mathrm{d}}$ of $0.25 \mathrm{~mL} / \mathrm{g}$ was calculated (for $10^{\circ} \mathrm{C}$ ). A series of field and laboratory investigations were conducted at a site in Borden, Ontario, to evaluate the transport of a halogenated hydrocarbon compounds (Makay et al. 1986; Roberts et al. 1986; Curtis et al. 1986). The Borden aquifer material is a clean, well-sorted, fine-to-medium grained sand with an average organic carbon fraction of 0.0002 (range 0.0001 to 0.0009) (Mackay et al. 1986). Batch experiments conducted at $21^{\circ} \mathrm{C}$ resulted in a $\mathrm{K}_{\mathrm{d}}$ of $0.15 \mathrm{~mL} / \mathrm{g}$. After correcting for differences in temperature, retardation factors calculated with this $\mathrm{K}_{\mathrm{d}}$ value were in very good agreement with retardation factors determined from both temporal and spatial field data. Chin et al. (1988) measured a $\mathrm{K}_{\mathrm{d}}$ value for a soil collected from Michawye Michigan. This soil had a sandy character and a fractional organic carbon content of 0.0013 . The $\mathrm{K}_{\mathrm{d}}$ value was determined to be $0.542 \mathrm{~mL} / \mathrm{g}$ (presumably at room temperature).

Based on the data presented here, it is estimated that the most probable value for a $\mathrm{K}_{\mathrm{d}}$ for carbon tetrachloride adsorption onto Hanford sediment is $0.2 \mathrm{~mL} / \mathrm{g}$, with a range of approximately 0.1 to 0.6 .

\subsection{Suspended Sediment $K_{d}$ Values}

In addition to $\mathrm{K}_{d}$ values for groundwater and the vadose zone, $\mathrm{K}_{\mathrm{d}}$ values also are needed for Columbia River suspended sediment. At this time, no measurements of $\mathrm{K}_{\mathrm{d}}$ values for Columbia River suspended sediment are available. The suspended sediment will be significantly different than typical Hanford sediment. The suspended sediment will consist primarily of silt and clay size material and have a significantly higher organic carbon content. Due to the lack of measured suspended sediment $\mathrm{K}_{\mathrm{d}}$ values, an estimation technique was used. The low, median and upper limit suspended sediment $\mathrm{K}_{\mathrm{d}}$ values for $\mathrm{I}(\mathrm{I}), \mathrm{U}(\mathrm{VI}), \mathrm{Sr}(\mathrm{II}), \mathrm{Cs}(\mathrm{I}), \mathrm{Pu}$ (valence unspecified), were estimated to be 1, 10, and 100x the values determined for Hanford sediment/groundwater. The mean $\mathrm{K}_{d}$ values determined for $\mathrm{Cr}(\mathrm{VI})$, and $\mathrm{Tc}(\mathrm{VII})$ for Hanford sediment/groundwater are zero, therefore the suspended sediment $\mathrm{K}_{\mathrm{d}}$ values were estimated as 1 , 10 , and 100x the maximum $\mathrm{K}_{\mathrm{d}}$ values determined for Hanford sediment/groundwater. For carbon tetrachloride, the median $K_{d}$ value was estimated using Equation 4:

$$
\mathrm{K}_{\mathrm{d}}=\mathrm{f}_{\mathrm{oc}} \times \mathrm{K}_{\mathrm{oc}}
$$

where $\mathrm{f}_{\mathrm{oc}}=$ the mass fraction organic matter

$\mathrm{K}_{\mathrm{oc}}=$ the distribution coefficient for organic carbon.

$\mathrm{K}_{\mathrm{oc}}$ was assumed to be $136 \mathrm{~mL} / \mathrm{g}$ (the average from Table 1, Truex et al. 2001) and $\mathrm{f}_{\mathrm{oc}}$ was assumed to be 0.01 . Using these values, the median suspended sediment $K_{d}$ value for carbon tetrachloride was estimated to be $1.4 \mathrm{~mL} / \mathrm{g}$. The minimum values are assumed to be $0.14 \mathrm{~g} / \mathrm{ml}$ and the maximum is assumed to be $2.8 \mathrm{~mL} / \mathrm{g}$. 


\subsection{Guidance for Selecting $K_{d}$ Values from $K_{d}$ Database}

In order to select a $K_{d}$ value that is sufficiently accurate for transport modeling purposes, careful consideration must be given to the $K_{d}$ values available for Hanford sediment listed in Tables 3 through 17 and the corresponding conditions under which these values were determined (Appendices $\mathrm{A}$ and $\mathrm{B}$ ). The specific set of conditions for which a particular $\mathrm{K}_{\mathrm{d}}$ value is to be applied should be matched as closely as possible to the experimental conditions used for determining the selected $\mathrm{K}_{\mathrm{d}}$ value. In general (for most $<2 \mathrm{~mm}$ Hanford formation sediment), differences in the chemical properties of the solution influence the $\mathrm{K}_{\mathrm{d}}$ values to a much greater extent than do differences in sediment properties. In addition, fewer data have been collected that are suitable for evaluating differences in $\mathrm{K}_{\mathrm{d}}$ values among different geologic units at Hanford (e.g., Hanford, Plio-Pleistocene, Ringold). As a result, more effort should be focused on the evaluation of the impact of solution chemical factors when selecting $K_{d}$ values. When attempting to select an appropriate $K_{d}$ value, greater reliability should be placed on values that have been measured multiple times, preferably in separate studies. In some cases, suspect outliers should be excluded from consideration, as was done in the case of uranium in this report.

Because different chemical parameters will affect the adsorption properties of individual contaminants differently; it is important that an individual with expert geochemical knowledge and experience in working with and determining $\mathrm{K}_{\mathrm{d}}$ values be used to evaluate and select appropriate $\mathrm{K}_{\mathrm{d}}$ values for transport modeling. Because $\mathrm{K}_{\mathrm{d}}$ values are available for only a limited set of conditions, a certain amount of interpolation and extrapolation may be required to select $K_{d}$ values for all conditions required for the transport models.

$\mathrm{K}_{\mathrm{d}}$ measurements are generally conducted on Hanford sediment material that is $<2 \mathrm{~mm}$ in size. For materials that contain significant amounts of gravel, $\mathrm{K}_{\mathrm{d}}$ values will be lower than those determined with $<2 \mathrm{~mm}$ size material because the surface area and corresponding quantity of adsorption sites is much

lower. For high gravel content material, often present at Hanford, especially near the Columbia River, it is necessary to make corrections to $\mathrm{K}_{\mathrm{d}}$ values determined with $<2 \mathrm{~mm}$ size material. For high $\mathrm{K}_{\mathrm{d}}$ contaminants (Cs, $\mathrm{Sr}$, and $\mathrm{Pu}$ ), Equation 5 is recommended (see Appendix A, Kaplan and Serne 2000).

$$
\mathrm{Kd}_{\mathrm{gc}}=(1-\mathrm{f}) \mathrm{Kd}_{<2 \mathrm{~mm}}+(\mathrm{f}) 0.23 \mathrm{Kd}_{<2 \mathrm{~mm}}
$$

where $\quad \mathrm{Kd}_{\mathrm{gc}}=$ the gravel corrected $\mathrm{Kd}$ value

$\mathrm{f}=$ the weight fraction gravel

$\mathrm{Kd}_{<2 \mathrm{~mm}}=$ the $\mathrm{K}_{\mathrm{d}}$ value determined using $<2 \mathrm{~mm}$ material.

For low $\mathrm{K}_{\mathrm{d}}$ contaminants, the following equation is recommended

$$
\mathrm{Kd}_{\mathrm{gc}}=(1-\mathrm{f}) \mathrm{Kd}_{<2 \mathrm{~mm}}
$$




\subsection{Data Gaps, Technical Needs, and Recommendations}

In general, the amount of error associated with a particular $\mathrm{K}_{\mathrm{d}}$ value (on a percentage basis) that is acceptable increases as the $K_{d}$ value increases. This is because contaminants with low $K_{d}$ values are the most mobile and as a result are more likely to be associated with greater risk. Large errors associated with high $\mathrm{K}_{\mathrm{d}}$ values will make a smaller difference from a risk perspective because the contaminant is not very mobile anyway, whereas large variations associated with moderate or low $\mathrm{K}_{\mathrm{d}}$ values can make the difference between whether the contaminant will be a significant risk driver or not.

Based on this rationale, the most beneficial future enhancements to the $\mathrm{K}_{\mathrm{d}}$ database would be obtained by focusing on the contaminants with low $\mathrm{K}_{\mathrm{d}}$ values [Cr(VI), I(I), $\mathrm{NO}_{3}{ }^{-}, \mathrm{Np}(\mathrm{V}), \mathrm{Se}(\mathrm{VI})$, $\mathrm{Tc}(\mathrm{VII})$, and U(VI)]. This list can be reduced further with other considerations, such as need, relative quality and quantity of available data, and other risk factors such as drinking water standards and quantity of the contaminants released at Hanford. Based upon the available data, it appears that the greatest need for further $\mathrm{K}_{\mathrm{d}}$ value determinations are for these low $\mathrm{K}_{\mathrm{d}}$ contaminants under the combined conditions of high $\mathrm{pH}$ and high salt. Also, consideration should be given to designing the experiments to reduce standard error as much as practical. A second data gap is the lack of $K_{d}$ values for sediment from the deeper unsaturated and saturated zones within the Plio-Pleistocene and Ringold Formations. Data for contaminant waste solution types is almost non-existent for these sediment types. No measured $\mathrm{K}_{\mathrm{d}}$ values are available for Columbia River suspended sediment. The range of the estimated $\mathrm{K}_{\mathrm{d}}$ values for suspended sediment could be greatly reduced by conducting measurements with actual material.

\subsection{Discussion}

A large body of $\mathrm{K}_{\mathrm{d}}$ data has been collected for radionuclides and toxic compounds that have the greatest potential for driving risk to human health and safety in the vadose zone and groundwater at the Hanford Site. In addition to the actual $\mathrm{K}_{\mathrm{d}}$ values, all significant experimental parameters and solution and sediment characterization data associated with these $\mathrm{K}_{\mathrm{d}}$ values have been compiled and documented as part of this task.

In general, with proper data selection and application to appropriate conditions, the linear adsorption model $\left(\mathrm{K}_{\mathrm{d}}\right.$ model) approach will be adequate for modeling transport through the Hanford system, especially for the far field where geochemical conditions remain fairly constant and contaminant loading of the adsorption sites is low. However, in some situations such as where large changes in chemical conditions occur (underneath a leaking high-level waste tank for example), the linear adsorption model will not be appropriate. In such cases, rapidly changing chemical conditions will result in large changes in reactivity of the contaminants with surface sites and result in large changes in $\mathrm{K}_{\mathrm{d}}$ values. In other words, $\mathrm{K}_{\mathrm{d}}$ values are significantly dependent on multiple chemical parameters such as $\mathrm{pH}$, sodium ion concentration, or contaminant concentration. High concentrations of contaminants have the potential to 
saturate adsorptions sites. An inherent assumption of the linear adsorption model approach is that the adsorption sites never become saturated. These limitations of the linear adsorption model approach have been dramatically illustrated by the recent work conducted on Cs(I) adsorption on Hanford sediment under conditions expected underneath a leaking high-level waste tank (Zachara et al. 2002).

When modeling adsorption from solutions whose compositions exceed the empirical range applicable for available $K_{d}$ measurements, additional measurements should be made to broaden the applicable range. In other cases, where the solution compositions and/or other adsorption parameters are not appropriate for the $\mathrm{K}_{\mathrm{d}}$ model approach, a more sophisticated (mechanistic) adsorption model should be considered.

Future revisions to this document/database are anticipated as new data become available. General guidance was provided to assist in the selection of appropriate $K_{d}$ values. This included appropriate matching of solution chemistry with the chemical condition used in the $\mathrm{K}_{\mathrm{d}}$ determinations. The effect of the differences in sediment mineralogy was indicated to be secondary to differences in solution chemistry with regard to impact of $\mathrm{K}_{\mathrm{d}}$ values. Guidance on how to make corrections for gravel content was also provided. The importance of using a qualified geochemist for selecting appropriate $\mathrm{K}_{\mathrm{d}}$ was stressed.

Table 3. Contaminant Distribution Coefficient Data for Americium

\begin{tabular}{|c|c|c|c|c|c|c|c|c|c|}
\hline Species & $\begin{array}{c}\text { Aqueous } \\
\text { Phase }\end{array}$ & Solid Phase & Method & $\begin{array}{c}\mathrm{Kd} \\
(\mathrm{mL} / \mathrm{g})\end{array}$ & Std. Dev. & $\begin{array}{l}\text { Equil. } \\
\text { Time }\end{array}$ & $\begin{array}{l}\text { In. Soln. } \\
\text { Conc. }\end{array}$ & $\begin{array}{l}\text { Solution/ } \\
\text { Solid } \\
(\mathrm{mL} / \mathrm{g})\end{array}$ & Reference \\
\hline Am(III) & $\begin{array}{l}0.002 \mathrm{M} \\
\mathrm{Ca}\left(\mathrm{NO}_{3}\right)_{2}\end{array}$ & Burbank Sand & Batch & $>1200$ & & 1 day & Trace & 2.5 & $(a, b)$ \\
\hline Am(III) & $\begin{array}{l}0.02 \mathrm{M} \\
\mathrm{Ca}\left(\mathrm{NO}_{3}\right)_{2}\end{array}$ & Burbank Sand & Batch & $>1200$ & & 1 day & Trace & 2.5 & $(a, b)$ \\
\hline Am(III) & $\begin{array}{l}0.05 \mathrm{M} \\
\mathrm{Ca}\left(\mathrm{NO}_{3}\right)_{2}\end{array}$ & Burbank Sand & Batch & $>1200$ & & 1 day & Trace & 2.5 & $(a, b)$ \\
\hline Am(III) & $\begin{array}{l}0.10 \mathrm{M} \\
\mathrm{Ca}\left(\mathrm{NO}_{3}\right)_{2}\end{array}$ & Burbank Sand & Batch & $>1200$ & & 1 day & Trace & 2.5 & $(a, b)$ \\
\hline Am(III) & $\begin{array}{l}0.20 \mathrm{M} \\
\mathrm{Ca}\left(\mathrm{NO}_{3}\right)_{2}\end{array}$ & Burbank Sand & Batch & $>1200$ & & 1 day & Trace & 2.5 & $(a, b)$ \\
\hline $\mathrm{Am}(\mathrm{III})$ & Soln 1 & Sed L & Batch & 4.02 & & 7 days & Trace & 5 & (c) \\
\hline Am(III) & Soln 2 & Sed L & Batch & 2.85 & & 7 days & Trace & 5 & (c) \\
\hline $\operatorname{Am}(\mathrm{III})$ & Soln 3 & Sed L & Batch & 148 & & 7 days & Trace & 5 & (c) \\
\hline $\mathrm{Am}(\mathrm{III})$ & Soln 4 & Sed L & Batch & 7.79 & & 7 days & Trace & 5 & (c) \\
\hline $\mathrm{Am}(\mathrm{III})$ & Soln 5 & Sed L & Batch & $>153$ & & 7 days & Trace & 5 & (c) \\
\hline $\operatorname{Am}(\mathrm{III})$ & Soln 6 & Sed L & Batch & $>1080$ & & 7 days & Trace & 5 & (c) \\
\hline Am(III) & Soln 7 & Sed L & Batch & 337 & & 7 days & Trace & 5 & (c) \\
\hline Am(III) & Soln 8 & Sed L & Batch & 99.6 & & 7 days & Trace & 5 & (c) \\
\hline Am(III) & Soln 9 & Sed L & Batch & 1.11 & & 7 days & Trace & 5 & (c) \\
\hline $\mathrm{Am}(\mathrm{III})$ & Soln 10 & Sed L & Batch & 3.25 & & 7 days & Trace & 5 & (c) \\
\hline $\mathrm{Am}(\mathrm{III})$ & Soln 11 & Sed L & Batch & 15.93 & & 7 days & Trace & 5 & (c) \\
\hline Am(III) & Soln 12 & Sed L & Batch & 6.87 & & 7 days & Trace & 5 & (c) \\
\hline
\end{tabular}




\begin{tabular}{|c|c|c|c|c|c|c|c|c|c|}
\hline Species & $\begin{array}{c}\text { Aqueous } \\
\text { Phase } \\
\end{array}$ & Solid Phase & Method & $\begin{array}{c}\mathrm{Kd} \\
(\mathrm{mL} / \mathrm{g}) \\
\end{array}$ & Std. Dev. & $\begin{array}{c}\text { Equil. } \\
\text { Time }\end{array}$ & $\begin{array}{c}\text { In. Soln. } \\
\text { Conc. }\end{array}$ & $\begin{array}{c}\text { Solution/ } \\
\text { Solid } \\
(\mathrm{mL} / \mathrm{g}) \\
\end{array}$ & Reference \\
\hline Am(III) & Soln 13 & Sed L & Batch & 3.22 & & 7 days & Trace & 5 & (c) \\
\hline Am(III) & Soln 14 & Sed L & Batch & $>1730$ & & 7 days & Trace & 5 & (c) \\
\hline Am(III) & Soln 15 & Sed L & Batch & 48.6 & & 7 days & Trace & 5 & (c) \\
\hline Am(III) & Soln 16 & Sed L & Batch & 3.43 & & 7 days & Trace & 5 & (c) \\
\hline Am(III) & Soln 17 & Sed L & Batch & 2.03 & & 7 days & Trace & 5 & (c) \\
\hline Am(III) & Soln 18 & Sed L & Batch & 2.95 & & 7 days & Trace & 5 & (c) \\
\hline Am(III) & Soln 19 & Sed L & Batch & 9.52 & & 7 days & Trace & 5 & (c) \\
\hline $\mathrm{Am}(\mathrm{III})$ & Soln 20 & Sed L & Batch & $>470$ & & 7 days & Trace & 5 & (c) \\
\hline $\mathrm{Am}(\mathrm{III})$ & Soln 1 & Sed P & Batch & 4.4 & & 7 days & Trace & 5 & (c) \\
\hline Am(III) & Soln 2 & Sed P & Batch & 2.2 & & 7 days & Trace & 5 & (c) \\
\hline Am(III) & Soln 3 & Sed P & Batch & 119 & & 7 days & Trace & 5 & (c) \\
\hline $\mathrm{Am}(\mathrm{III})$ & Soln 4 & Sed P & Batch & 6.68 & & 7 days & Trace & 5 & (c) \\
\hline Am(III) & Soln 5 & Sed P & Batch & $>169$ & & 7 days & Trace & 5 & (c) \\
\hline Am(III) & Soln 6 & Sed P & Batch & $>1670$ & & 7 days & Trace & 5 & (c) \\
\hline Am(III) & Soln 7 & Sed P & Batch & 288 & & 7 days & Trace & 5 & (c) \\
\hline Am(III) & Soln 8 & Sed P & Batch & 66.2 & & 7 days & Trace & 5 & (c) \\
\hline Am(III) & Soln 9 & Sed P & Batch & 0.97 & & 7 days & Trace & 5 & (c) \\
\hline Am(III) & Soln 10 & Sed P & Batch & 4.25 & & 7 days & Trace & 5 & (c) \\
\hline $\mathrm{Am}(\mathrm{III})$ & Soln 11 & Sed P & Batch & 10.1 & & 7 days & Trace & 5 & (c) \\
\hline Am(III) & Soln 12 & Sed P & Batch & 5.99 & & 7 days & Trace & 5 & (c) \\
\hline Am(III) & Soln 13 & Sed P & Batch & 2.43 & & 7 days & Trace & 5 & (c) \\
\hline Am(III) & Soln 14 & Sed P & Batch & 306 & & 7 days & Trace & 5 & (c) \\
\hline Am(III) & Soln 15 & Sed P & Batch & 41 & & 7 days & Trace & 5 & (c) \\
\hline Am(III) & Soln 16 & Sed P & Batch & 4.13 & & 7 days & Trace & 5 & (c) \\
\hline Am(III) & Soln 17 & Sed P & Batch & 2.01 & & 7 days & Trace & 5 & (c) \\
\hline $\mathrm{Am}(\mathrm{III})$ & Soln 18 & Sed P & Batch & 2.76 & & 7 days & Trace & 5 & (c) \\
\hline Am(III) & Soln 19 & Sed P & Batch & 7.5 & & 7 days & Trace & 5 & (c) \\
\hline $\mathrm{Am}(\mathrm{III})$ & Soln 20 & Sed P & Batch & $>520$ & & 7 days & Trace & 5 & (c) \\
\hline Am(III) & Soln 1 & Sed S & Batch & 28.6 & & 7 days & Trace & 5 & (c) \\
\hline Am(III) & Soln 2 & Sed S & Batch & 5.91 & & 7 days & Trace & 5 & (c) \\
\hline Am(III) & Soln 3 & Sed S & Batch & $>3160$ & & 7 days & Trace & 5 & (c) \\
\hline Am(III) & Soln 4 & Sed S & Batch & 28.5 & & 7 days & Trace & 5 & (c) \\
\hline Am(III) & Soln 5 & Sed S & Batch & $>133$ & & 7 days & Trace & 5 & (c) \\
\hline Am(III) & Soln 6 & Sed S & Batch & $>1300$ & & 7 days & Trace & 5 & (c) \\
\hline Am(III) & Soln 7 & Sed S & Batch & 439 & & 7 days & Trace & 5 & (c) \\
\hline Am(III) & Soln 8 & Sed S & Batch & 380 & & 7 days & Trace & 5 & (c) \\
\hline $\mathrm{Am}(\mathrm{III})$ & Soln 9 & Sed S & Batch & 2.47 & & 7 days & Trace & 5 & (c) \\
\hline Am(III) & Soln 10 & Sed S & Batch & 10.8 & & 7 days & Trace & 5 & (c) \\
\hline Am(III) & Soln 11 & Sed S & Batch & 72 & & 7 days & Trace & 5 & (c) \\
\hline
\end{tabular}




\begin{tabular}{|c|c|c|c|c|c|c|c|c|c|}
\hline Species & $\begin{array}{c}\text { Aqueous } \\
\text { Phase }\end{array}$ & Solid Phase & Method & $\begin{array}{c}\mathrm{Kd} \\
(\mathrm{mL} / \mathrm{g})\end{array}$ & Std. Dev. & $\begin{array}{c}\text { Equil. } \\
\text { Time }\end{array}$ & $\begin{array}{c}\text { In. Soln. } \\
\text { Conc. }\end{array}$ & $\begin{array}{c}\text { Solution/ } \\
\text { Solid } \\
(\mathrm{mL} / \mathrm{g})\end{array}$ & Reference \\
\hline Am(III) & Soln 12 & Sed S & Batch & 12 & & 7 days & Trace & 5 & (c) \\
\hline Am(III) & Soln 13 & Sed S & Batch & 5.7 & & 7 days & Trace & 5 & (c) \\
\hline Am(III) & Soln 14 & Sed S & Batch & $>2350$ & & 7 days & Trace & 5 & (c) \\
\hline Am(III) & Soln 15 & Sed S & Batch & 187 & & 7 days & Trace & 5 & (c) \\
\hline $\mathrm{Am}(\mathrm{III})$ & Soln 16 & Sed S & Batch & 10.4 & & 7 days & Trace & 5 & (c) \\
\hline $\mathrm{Am}(\mathrm{III})$ & Soln 17 & Sed S & Batch & 4.68 & & 7 days & Trace & 5 & (c) \\
\hline $\mathrm{Am}(\mathrm{III})$ & Soln 18 & Sed S & Batch & 9.45 & & 7 days & Trace & 5 & (c) \\
\hline $\mathrm{Am}(\mathrm{III})$ & Soln 19 & Sed S & Batch & 24.6 & & 7 days & Trace & 5 & (c) \\
\hline Am(III) & Soln 20 & Sed S & Batch & $>481$ & & 7 days & Trace & 5 & (c) \\
\hline Am(III) & $\begin{array}{l}\text { AAW, } \mathrm{pH} \\
1.7^{*}\end{array}$ & 299-W18-6, -7 & Batch & $<1$ & & & & & (d) \\
\hline $\mathrm{Am}(\mathrm{III})$ & AAW, pH 2 & 299-W18-6, -7 & Batch & $<1$ & & & & & (d) \\
\hline Am(III) & AAW, pH 3 & 299-W18-6, -7 & Batch & $<1$ & & & & & (d) \\
\hline \multicolumn{10}{|c|}{\begin{tabular}{|l} 
(a) Rouston et al. 1975. \\
(b) Rouston et al. 1976. \\
(c) Delegard and Barney 1983. \\
(d) Hajek and Knoll 1966. \\
*Data is best estimate.
\end{tabular}} \\
\hline
\end{tabular}


Table 4. Contaminant Distribution Coefficient Data for Cobalt

\begin{tabular}{|c|c|c|c|c|c|c|c|c|c|}
\hline Species & $\begin{array}{l}\text { Aqueous } \\
\text { Phase }\end{array}$ & Solid Phase & Method & $\begin{array}{c}\mathrm{Kd} \\
(\mathrm{mL} / \mathrm{g})\end{array}$ & Std. Dev. & $\begin{array}{l}\text { Equil. } \\
\text { Time }\end{array}$ & $\begin{array}{l}\text { In. Soln. } \\
\text { Conc. }\end{array}$ & $\begin{array}{l}\text { Soln/Solid } \\
(\mathrm{mL} / \mathrm{g})\end{array}$ & Reference \\
\hline $\mathrm{Co}(\mathrm{II})$ & Hanford GW & Trench-8 & Batch & $1.25 \mathrm{E}+04$ & $6.00 \mathrm{E}+02$ & 5 days & Trace & 30 & (a) \\
\hline $\mathrm{Co}(\mathrm{II})$ & Hanford GW & Trench-8 & Batch & $1.16 \mathrm{E}+04$ & $6.00 \mathrm{E}+02$ & 44 days & Trace & 30 & (a) \\
\hline $\mathrm{Co}(\mathrm{II})$ & Solution 1 & Soil B & Batch & 4570 & 650 & 7 days & Trace & 30 & (b) \\
\hline $\mathrm{Co}(\mathrm{II})$ & Solution 2 & Soil B & Batch & 2750 & 200 & 7 days & Trace & 30 & (b) \\
\hline $\mathrm{Co}(\mathrm{II})$ & Solution 3 & Soil B & Batch & 3670 & 160 & 7 days & Trace & 30 & (b) \\
\hline $\mathrm{Co}(\mathrm{II})$ & Solution 4 & Soil B & Batch & 11070 & 6480 & 7 days & Trace & 30 & (b) \\
\hline $\mathrm{Co}(\mathrm{II})$ & Solution 5 & Soil B & Batch & 2735 & 490 & 7 days & Trace & 30 & (b) \\
\hline $\mathrm{Co}(\mathrm{II})$ & Solution 6 & Soil B & Batch & 4090 & 620 & 7 days & Trace & 30 & (b) \\
\hline $\mathrm{Co}(\mathrm{II})$ & Soln 1 & Sed L & Batch & -0.35 & & 7 days & Trace & 5 & (c) \\
\hline $\mathrm{Co}(\mathrm{II})$ & Soln 2 & Sed L & Batch & -0.02 & & 7 days & Trace & 5 & (c) \\
\hline $\mathrm{Co}(\mathrm{II})$ & Soln 3 & Sed L & Batch & 20.4 & & 7 days & Trace & 5 & (c) \\
\hline $\mathrm{Co}(\mathrm{II})$ & Soln 4 & Sed L & Batch & 0.404 & & 7 days & Trace & 5 & (c) \\
\hline $\mathrm{Co}(\mathrm{II})$ & Soln 5 & Sed L & Batch & 3.13 & & 7 days & Trace & 5 & (c) \\
\hline $\mathrm{Co}(\mathrm{II})$ & Soln 6 & Sed L & Batch & 0.927 & & 7 days & Trace & 5 & (c) \\
\hline $\mathrm{Co}(\mathrm{II})$ & Soln 7 & Sed L & Batch & 1.96 & & 7 days & Trace & 5 & (c) \\
\hline $\mathrm{Co}(\mathrm{II})$ & Soln 8 & Sed L & Batch & 5.79 & & 7 days & Trace & 5 & (c) \\
\hline $\mathrm{Co}(\mathrm{II})$ & Soln 9 & Sed L & Batch & 0.257 & & 7 days & Trace & 5 & (c) \\
\hline $\mathrm{Co}(\mathrm{II})$ & Soln 10 & Sed L & Batch & 0.248 & & 7 days & Trace & 5 & (c) \\
\hline $\mathrm{Co}(\mathrm{II})$ & Soln 11 & Sed L & Batch & -0.078 & & 7 days & Trace & 5 & (c) \\
\hline $\mathrm{Co}(\mathrm{II})$ & Soln 12 & Sed L & Batch & -0.169 & & 7 days & Trace & 5 & (c) \\
\hline $\mathrm{Co}(\mathrm{II})$ & Soln 13 & Sed L & Batch & -0.168 & & 7 days & Trace & 5 & (c) \\
\hline $\mathrm{Co}(\mathrm{II})$ & Soln 14 & Sed L & Batch & 2.97 & & 7 days & Trace & 5 & (c) \\
\hline $\mathrm{Co}(\mathrm{II})$ & Soln 15 & Sed L & Batch & 23.4 & & 7 days & Trace & 5 & (c) \\
\hline $\mathrm{Co}(\mathrm{II})$ & Soln 16 & Sed L & Batch & 0.382 & & 7 days & Trace & 5 & (c) \\
\hline $\mathrm{Co}(\mathrm{II})$ & Soln 17 & Sed L & Batch & 0.116 & & 7 days & Trace & 5 & (c) \\
\hline $\mathrm{Co}(\mathrm{II})$ & Soln 18 & Sed L & Batch & 1.45 & & 7 days & Trace & 5 & (c) \\
\hline $\mathrm{Co}(\mathrm{II})$ & Soln 19 & Sed L & Batch & 0.251 & & 7 days & Trace & 5 & (c) \\
\hline $\mathrm{Co}(\mathrm{II})$ & Soln 20 & Sed L & Batch & 1.1 & & 7 days & Trace & 5 & (c) \\
\hline $\mathrm{Co}(\mathrm{II})$ & Soln 1 & Sed P & Batch & -0.391 & & 7 days & Trace & 5 & (c) \\
\hline $\mathrm{Co}(\mathrm{II})$ & Soln 2 & Sed P & Batch & -0.409 & & 7 days & Trace & 5 & (c) \\
\hline $\mathrm{Co}(\mathrm{II})$ & Soln 3 & Sed P & Batch & 16.7 & & 7 days & Trace & 5 & (c) \\
\hline $\mathrm{Co}(\mathrm{II})$ & Soln 4 & Sed P & Batch & 0.782 & & 7 days & Trace & 5 & (c) \\
\hline $\mathrm{Co}(\mathrm{II})$ & Soln 5 & Sed P & Batch & 3.71 & & 7 days & Trace & 5 & (c) \\
\hline $\mathrm{Co}(\mathrm{II})$ & Soln 6 & Sed P & Batch & 1.44 & & 7 days & Trace & 5 & (c) \\
\hline
\end{tabular}




\begin{tabular}{|c|c|c|c|c|c|c|c|c|c|}
\hline Species & $\begin{array}{l}\text { Aqueous } \\
\text { Phase }\end{array}$ & Solid Phase & Method & $\begin{array}{c}\mathrm{Kd} \\
(\mathrm{mL} / \mathrm{g})\end{array}$ & Std. Dev. & $\begin{array}{l}\text { Equil. } \\
\text { Time }\end{array}$ & $\begin{array}{c}\text { In. Soln. } \\
\text { Conc. }\end{array}$ & $\begin{array}{c}\text { Soln/Solid } \\
(\mathrm{mL} / \mathrm{g})\end{array}$ & Reference \\
\hline $\mathrm{Co}(\mathrm{II})$ & Soln 7 & Sed P & Batch & 2.02 & & 7 days & Trace & 5 & (c) \\
\hline $\mathrm{Co}(\mathrm{II})$ & Soln 8 & Sed P & Batch & 4.54 & & 7 days & Trace & 5 & (c) \\
\hline $\mathrm{Co}(\mathrm{II})$ & Soln 9 & Sed P & Batch & 0.122 & & 7 days & Trace & 5 & (c) \\
\hline $\mathrm{Co}(\mathrm{II})$ & Soln 10 & Sed P & Batch & 0.298 & & 7 days & Trace & 5 & (c) \\
\hline $\mathrm{Co}(\mathrm{II})$ & Soln 11 & Sed P & Batch & 0.139 & & 7 days & Trace & 5 & (c) \\
\hline $\mathrm{Co}(\mathrm{II})$ & Soln 12 & Sed P & Batch & -0.21 & & 7 days & Trace & 5 & (c) \\
\hline $\mathrm{Co}(\mathrm{II})$ & Soln 13 & Sed P & Batch & -0.049 & & 7 days & Trace & 5 & (c) \\
\hline $\mathrm{Co}(\mathrm{II})$ & Soln 14 & Sed P & Batch & 2.58 & & 7 days & Trace & 5 & (c) \\
\hline $\mathrm{Co}(\mathrm{II})$ & Soln 15 & Sed P & Batch & 21.9 & & 7 days & Trace & 5 & (c) \\
\hline $\mathrm{Co}(\mathrm{II})$ & Soln 16 & Sed P & Batch & 0.574 & & 7 days & Table & 5 & (c) \\
\hline $\mathrm{Co}(\mathrm{II})$ & Soln 17 & Sed P & Batch & 0.183 & & 7 days & Trace & 5 & (c) \\
\hline $\mathrm{Co}(\mathrm{II})$ & Soln 18 & Sed P & Batch & 1.68 & & 7 days & Trace & 5 & (c) \\
\hline $\mathrm{Co}(\mathrm{II})$ & Soln 19 & Sed P & Batch & -0.033 & & 7 days & Trace & 5 & (c) \\
\hline $\mathrm{Co}(\mathrm{II})$ & Soln 20 & Sed P & Batch & 0.915 & & 7 days & Trace & 5 & (c) \\
\hline $\mathrm{Co}(\mathrm{II})$ & Soln 1 & Sed S & Batch & -0.057 & & 7 days & Trace & 5 & (c) \\
\hline $\mathrm{Co}(\mathrm{II})$ & Soln 2 & Sed S & Batch & 0.095 & & 7 days & Trace & 5 & (c) \\
\hline $\mathrm{Co}(\mathrm{II})$ & Soln 3 & Sed S & Batch & 14.8 & & 7 days & Trace & 5 & (c) \\
\hline $\mathrm{Co}(\mathrm{II})$ & Soln 4 & Sed S & Batch & 1.21 & & 7 days & Trace & 5 & (c) \\
\hline $\mathrm{Co}(\mathrm{II})$ & Soln 5 & Sed S & Batch & 2.41 & & 7 days & Trace & 5 & (c) \\
\hline $\mathrm{Co}(\mathrm{II})$ & Soln 6 & Sed S & Batch & 0.786 & & 7 days & Trace & 5 & (c) \\
\hline $\mathrm{Co}(\mathrm{II})$ & Soln 7 & Sed S & Batch & 1.95 & & 7 days & Trace & 5 & (c) \\
\hline $\mathrm{Co}(\mathrm{II})$ & Soln 8 & Sed S & Batch & 3.77 & & 7 days & Trace & 5 & (c) \\
\hline $\mathrm{Co}(\mathrm{II})$ & Soln 9 & Sed S & Batch & 0.121 & & 7 days & Trace & 5 & (c) \\
\hline $\mathrm{Co}(\mathrm{II})$ & Soln 10 & Sed S & Batch & -0.036 & & 7 days & Trace & 5 & (c) \\
\hline $\mathrm{Co}(\mathrm{II})$ & Soln 11 & Sed S & Batch & 0.366 & & 7 days & Trace & 5 & (c) \\
\hline $\mathrm{Co}(\mathrm{II})$ & Soln 12 & Sed S & Batch & -0.053 & & 7 days & Trace & 5 & (c) \\
\hline $\mathrm{Co}(\mathrm{II})$ & Soln 13 & Sed S & Batch & -0.025 & & 7 days & Trace & 5 & (c) \\
\hline $\mathrm{Co}(\mathrm{II})$ & Soln 14 & Sed S & Batch & 2.84 & & 7 days & Trace & 5 & (c) \\
\hline $\mathrm{Co}(\mathrm{II})$ & Soln 15 & Sed S & Batch & 13.3 & & 7 days & Trace & 5 & (c) \\
\hline $\mathrm{Co}(\mathrm{II})$ & Soln 16 & Sed S & Batch & 0.572 & & 7 days & Trace & 5 & (c) \\
\hline $\mathrm{Co}(\mathrm{II})$ & Soln 17 & Sed S & Batch & 0.102 & & 7 days & Trace & 5 & (c) \\
\hline $\mathrm{Co}(\mathrm{II})$ & Soln 18 & Sed S & Batch & 0.723 & & 7 days & Trace & 5 & (c) \\
\hline $\mathrm{Co}(\mathrm{II})$ & Soln 19 & Sed S & Batch & -0.079 & & 7 days & Trace & 5 & (c) \\
\hline $\mathrm{Co}(\mathrm{II})$ & Soln 20 & Sed S & Batch & 1.22 & & 7 days & Trace & 5 & (c) \\
\hline $\mathrm{Co}(\mathrm{II})$ & HGW3 & 200-BP-1 & Batch & 2556 & 209 & 14 days & Trace & 30 & (d) \\
\hline $\operatorname{Co}(\mathrm{II})$ & $150 \mathrm{ppb} \mathrm{CN}$ & 200-BP-1 & Batch & 1.9 & 0.2 & 14 days & Trace & 30 & (d) \\
\hline $\mathrm{Co}(\mathrm{II})$ & $2000 \mathrm{ppb} \mathrm{CN}$ & 200-BP-1 & Batch & 0.03 & 0.06 & 14 days & Trace & 30 & (d) \\
\hline
\end{tabular}




\begin{tabular}{|c|c|c|c|c|c|c|c|c|c|}
\hline Species & $\begin{array}{l}\text { Aqueous } \\
\text { Phase }\end{array}$ & Solid Phase & Method & $\begin{array}{c}\mathrm{Kd} \\
(\mathrm{mL} / \mathrm{g})\end{array}$ & Std. Dev. & $\begin{array}{l}\text { Equil. } \\
\text { Time }\end{array}$ & $\begin{array}{c}\text { In. Soln. } \\
\text { Conc. }\end{array}$ & $\begin{array}{l}\text { Soln/Solid } \\
(\mathrm{mL} / \mathrm{g})\end{array}$ & Reference \\
\hline $\mathrm{Co}(\mathrm{II})$ & $25 \mathrm{ppb}$ EDTA & 200-BP-1 & Batch & 1071 & 138 & 14 days & Trace & 30 & (d) \\
\hline $\mathrm{Co}(\mathrm{II})$ & $\begin{array}{l}200 \mathrm{ppb} \\
\text { EDTA }\end{array}$ & 200-BP-1 & Batch & 72 & 7 & 14 days & Trace & 30 & (d) \\
\hline $\mathrm{Co}(\mathrm{II})$ & $\begin{array}{l}2000 \mathrm{ppb} \\
\text { EDTA }\end{array}$ & 200-BP-1 & Batch & 3 & 1 & 14 days & Trace & 30 & (d) \\
\hline \multicolumn{10}{|c|}{$\begin{array}{l}\text { (a) Serne et al. } 1993 . \\
\text { (b) Gee and Campbell } 1980 . \\
\text { (c) Delegard and Barney } 1983 . \\
\text { (d) Cantrell and Serne } 1993 .\end{array}$} \\
\hline
\end{tabular}


Table 5. Contaminant Distribution Coefficient Data for Cesium

\begin{tabular}{|c|c|c|c|c|c|c|c|c|c|}
\hline Species & $\begin{array}{l}\text { Aqueous } \\
\text { Phase }\end{array}$ & Solid Phase & Method & $\begin{array}{c}\mathrm{Kd} \\
(\mathrm{mL} / \mathrm{g})\end{array}$ & Std. Dev. & $\begin{array}{c}\text { Equil. } \\
\text { Time }\end{array}$ & $\begin{array}{c}\text { In. Soln. } \\
\text { Conc. }\end{array}$ & $\begin{array}{c}\text { Solution/ } \\
\text { Solid } \\
(\mathrm{mL} / \mathrm{g})\end{array}$ & Reference \\
\hline $\mathrm{Cs}(\mathrm{I})$ & Hanford GW & CGS-1 & Batch & 1270 & 36 & 4 days & Trace & 30 & (a) \\
\hline $\mathrm{Cs}(\mathrm{I})$ & Hanford GW & CGS-1 & Batch & 2190 & 414 & 14 days & Trace & 30 & (a) \\
\hline $\mathrm{Cs}(\mathrm{I})$ & Hanford GW & CGS-1 & Batch & 3120 & 630 & 35 days & Trace & 30 & (a) \\
\hline $\mathrm{Cs}(\mathrm{I})$ & $\begin{array}{l}\text { Acid Proc } \\
\text { Waste }\end{array}$ & CGS-1 & Batch & 1630 & 310 & 4 days & Trace & 30 & (a) \\
\hline $\mathrm{Cs}(\mathrm{I})$ & $\begin{array}{l}\text { Acid Proc } \\
\text { Waste }\end{array}$ & CGS-1 & Batch & 3120 & 520 & 14 days & Trace & 30 & (a) \\
\hline \multirow[t]{2}{*}{$\mathrm{Cs}(\mathrm{I})$} & $\begin{array}{l}\text { Acid Proc } \\
\text { Waste }\end{array}$ & CGS-1 & Batch & 3580 & 904 & 35 days & Trace & 30 & (a) \\
\hline & & & & & & & & 30 & (a) \\
\hline $\mathrm{Cs}(\mathrm{I})$ & Hanford GW & TBS-1 & Batch & 3720 & 760 & 4 days & Trace & 30 & (a) \\
\hline $\mathrm{Cs}(\mathrm{I})$ & Hanford GW & TBS-1 & Batch & 5110 & 690 & 14 days & Trace & 30 & (a) \\
\hline $\mathrm{Cs}(\mathrm{I})$ & Hanford GW & TBS-1 & Batch & 14000 & 1630 & 35 days & Trace & 30 & (a) \\
\hline $\mathrm{Cs}(\mathrm{I})$ & $\begin{array}{l}\text { Acid Proc } \\
\text { Waste }\end{array}$ & TBS-1 & Batch & 2330 & 420 & 4 days & Trace & 30 & (a) \\
\hline $\mathrm{Cs}(\mathrm{I})$ & $\begin{array}{l}\text { Acid Proc } \\
\text { Waste }\end{array}$ & TBS-1 & Batch & 3380 & 270 & 14 days & Trace & 30 & (a) \\
\hline $\mathrm{Cs}(\mathrm{I})$ & $\begin{array}{l}\text { Acid Proc } \\
\text { Waste }\end{array}$ & TBS-1 & Batch & 3180 & 130 & 35 days & Trace & 30 & (a) \\
\hline $\mathrm{Cs}(\mathrm{I})$ & Hanford GW & Trench-8 & Batch & 540 & 30 & 5 days & Trace & 30 & (a) \\
\hline $\mathrm{Cs}(\mathrm{I})$ & Hanford GW & Trench-8 & Batch & 690 & 80 & 44 days & Trace & 30 & (a) \\
\hline $\mathrm{Cs}(\mathrm{I})$ & Solution 1 & Soil A & Batch & 5307 & 100 & 8.5 days & Trace & 30 & (b) \\
\hline $\mathrm{Cs}(\mathrm{I})$ & Solution 2 & Soil A & Batch & 3377 & 1311 & 8.5 days & Trace & 30 & (b) \\
\hline $\mathrm{Cs}(\mathrm{I})$ & Solution 3 & Soil A & Batch & 3730 & 1387 & 8.5 days & Trace & 30 & (b) \\
\hline $\mathrm{Cs}(\mathrm{I})$ & Solution 4 & Soil A & Batch & 8832 & 3417 & 8.5 days & Trace & 30 & (b) \\
\hline $\mathrm{Cs}(\mathrm{I})$ & Solution 5 & Soil A & Batch & 5995 & 3632 & 8.5 days & Trace & 30 & (b) \\
\hline $\mathrm{Cs}(\mathrm{I})$ & Solution 6 & Soil A & Batch & 4233 & 1321 & 8.5 days & Trace & 30 & (b) \\
\hline $\mathrm{Cs}(\mathrm{I})$ & Solution 1 & Soil A & Batch & 13525 & 4741 & 21.5 days & Trace & 30 & (b) \\
\hline $\mathrm{Cs}(\mathrm{I})$ & Solution 2 & Soil A & Batch & 6432 & 1219 & 21.5 days & Trace & 30 & (b) \\
\hline $\mathrm{Cs}(\mathrm{I})$ & Solution 3 & Soil A & Batch & 7298 & 1098 & 21.5 days & Trace & 30 & (b) \\
\hline $\mathrm{Cs}(\mathrm{I})$ & Solution 4 & Soil A & Batch & 8217 & 2380 & 21.5 days & Trace & 30 & (b) \\
\hline $\mathrm{Cs}(\mathrm{I})$ & Solution 5 & Soil A & Batch & 6033 & 1534 & 21.5 days & Trace & 30 & (b) \\
\hline $\mathrm{Cs}(\mathrm{I})$ & Solution 6 & Soil A & Batch & 11522 & 2154 & 21.5 days & Trace & 30 & (b) \\
\hline $\mathrm{Cs}(\mathrm{I})$ & Solution 1 & Soil B & Batch & 2042 & 401 & 8.5 days & Trace & 30 & (b) \\
\hline
\end{tabular}




\begin{tabular}{|c|c|c|c|c|c|c|c|c|c|}
\hline Species & $\begin{array}{c}\text { Aqueous } \\
\text { Phase }\end{array}$ & Solid Phase & Method & $\begin{array}{c}\mathrm{Kd} \\
(\mathrm{mL} / \mathrm{g})\end{array}$ & Std. Dev. & $\begin{array}{c}\text { Equil. } \\
\text { Time }\end{array}$ & $\begin{array}{c}\text { In. Soln. } \\
\text { Conc. }\end{array}$ & $\begin{array}{l}\text { Solution/ } \\
\text { Solid } \\
(\mathrm{mL} / \mathrm{g})\end{array}$ & Reference \\
\hline $\mathrm{Cs}(\mathrm{I})$ & Solution 2 & Soil B & Batch & 1388 & 226 & 8.5 days & Trace & 30 & (b) \\
\hline $\mathrm{Cs}(\mathrm{I})$ & Solution 3 & Soil B & Batch & 2291 & 819 & 8.5 days & Trace & 30 & (b) \\
\hline $\mathrm{Cs}(\mathrm{I})$ & Solution 4 & Soil B & Batch & 3405 & 1215 & 8.5 days & Trace & 30 & (b) \\
\hline $\mathrm{Cs}(\mathrm{I})$ & Solution 5 & Soil B & Batch & 5708 & 2635 & 8.5 days & Trace & 30 & (b) \\
\hline $\mathrm{Cs}(\mathrm{I})$ & Solution 6 & Soil B & Batch & 3215 & 470 & 8.5 days & Trace & 30 & (b) \\
\hline $\mathrm{Cs}(\mathrm{I})$ & Solution 1 & Soil B & Batch & 1505 & 320 & 21.5 days & Trace & 30 & (b) \\
\hline $\mathrm{Cs}(\mathrm{I})$ & Solution 2 & Soil B & Batch & 1094 & 295 & 21.5 days & Trace & 30 & (b) \\
\hline $\mathrm{Cs}(\mathrm{I})$ & Solution 3 & Soil B & Batch & 3016 & 722 & 21.5 days & Trace & 30 & (b) \\
\hline $\mathrm{Cs}(\mathrm{I})$ & Solution 4 & Soil B & Batch & 5805 & 2109 & 21.5 days & Trace & 30 & (b) \\
\hline $\mathrm{Cs}(\mathrm{I})$ & Solution 5 & Soil B & Batch & 3045 & 377 & 21.5 days & Trace & 30 & (b) \\
\hline $\mathrm{Cs}(\mathrm{I})$ & Solution 6 & Soil B & Batch & 3253 & 80 & 21.5 days & Trace & 30 & (b) \\
\hline $\mathrm{Cs}(\mathrm{I})$ & Solution L & Soil B & Batch & 268 & 4 & 7 days & Trace & 30 & (b) \\
\hline $\mathrm{Cs}(\mathrm{I})$ & Solution H & Soil B & Batch & 25 & 1 & 7 days & Trace & 30 & (b) \\
\hline $\mathrm{Cs}(\mathrm{I})$ & Hanford GW2 & B850007A & Batch & 2045 & 275 & 14 days & Trace & 30 & (c) \\
\hline $\mathrm{Cs}(\mathrm{I})$ & Hanford GW2 & B850010A & Batch & 2090 & 305 & 14 days & Trace & 30 & (c) \\
\hline $\mathrm{Cs}(\mathrm{I})$ & Hanford GW2 & B850012A & Batch & 2024 & 305 & 14 days & Trace & 30 & (c) \\
\hline $\mathrm{Cs}(\mathrm{I})$ & Hanford GW2 & B850014A & Batch & 1970 & 40 & 14 days & Trace & 30 & (c) \\
\hline $\mathrm{Cs}(\mathrm{I})$ & Hanford GW2 & B850015A & Batch & 1502 & 80 & 14 days & Trace & 30 & (c) \\
\hline $\mathrm{Cs}(\mathrm{I})$ & Hanford GW2 & B850016A & Batch & 1536 & 270 & 14 days & Trace & 30 & (c) \\
\hline $\mathrm{Cs}(\mathrm{I})$ & Hanford GW2 & B850017A & Batch & 2267 & 388 & 14 days & Trace & 30 & (c) \\
\hline $\mathrm{Cs}(\mathrm{I})$ & Hanford GW2 & B850019A & Batch & 2862 & 396 & 14 days & Trace & 30 & (c) \\
\hline $\mathrm{Cs}(\mathrm{I})$ & Hanford GW2 & B850020A & Batch & 2252 & 292 & 14 days & Trace & 30 & (c) \\
\hline $\mathrm{Cs}(\mathrm{I})$ & Hanford GW2 & B850021A & Batch & 2073 & 409 & 14 days & Trace & 30 & (c) \\
\hline $\mathrm{Cs}(\mathrm{I})$ & Hanford GW2 & B850022A & Batch & 1374 & 332 & 14 days & Trace & 30 & (c) \\
\hline $\mathrm{Cs}(\mathrm{I})$ & Hanford GW2 & B850023A & Batch & 2295 & 280 & 14 days & Trace & 30 & (c) \\
\hline $\mathrm{Cs}(\mathrm{I})$ & Hanford GW2 & B850024A & Batch & 2213 & 455 & 14 days & Trace & 30 & (c) \\
\hline $\mathrm{Cs}(\mathrm{I})$ & Hanford GW2 & B850025A & Batch & 1717 & 484 & 14 days & Trace & 30 & (c) \\
\hline $\mathrm{Cs}(\mathrm{I})$ & Hanford GW2 & B850027A & Batch & 1563 & 259 & 14 days & Trace & 30 & (c) \\
\hline $\mathrm{Cs}(\mathrm{I})$ & Hanford GW2 & B850029A & Batch & 2129 & 423 & 14 days & Trace & 30 & (c) \\
\hline $\mathrm{Cs}(\mathrm{I})$ & Hanford GW2 & B850031A & Batch & 4068 & 259 & 14 days & Trace & 30 & (c) \\
\hline $\mathrm{Cs}(\mathrm{I})$ & Hanford GW2 & B850032A & Batch & 1792 & 315 & 14 days & Trace & 30 & (c) \\
\hline $\mathrm{Cs}(\mathrm{I})$ & Hanford GW2 & B850034A & Batch & 1897 & 178 & 14 days & Trace & 30 & (c) \\
\hline $\mathrm{Cs}(\mathrm{I})$ & Hanford GW2 & B850035A & Batch & 1435 & 133 & 14 days & Trace & 30 & (c) \\
\hline $\mathrm{Cs}(\mathrm{I})$ & $\begin{array}{l}\text { REDOX } \\
\text { Liquor }\end{array}$ & MSG-1 & Batch & 28 & & 4 days & Trace & 10 & (d) \\
\hline
\end{tabular}




\begin{tabular}{|c|c|c|c|c|c|c|c|c|c|}
\hline Species & $\begin{array}{l}\text { Aqueous } \\
\text { Phase }\end{array}$ & Solid Phase & Method & $\begin{array}{c}\mathrm{Kd} \\
(\mathrm{mL} / \mathrm{g})\end{array}$ & Std. Dev. & $\begin{array}{l}\text { Equil. } \\
\text { Time }\end{array}$ & $\begin{array}{l}\text { In. Soln. } \\
\text { Conc. }\end{array}$ & $\begin{array}{l}\text { Solution/ } \\
\text { Solid } \\
(\mathrm{mL} / \mathrm{g})\end{array}$ & Reference \\
\hline $\mathrm{Cs}(\mathrm{I})$ & $\begin{array}{l}\text { REDOX } \\
\text { Liquor }\end{array}$ & MSG-1 & Batch & 20 & & 4 days & Trace & 10 & (d) \\
\hline $\mathrm{Cs}(\mathrm{I})$ & $\begin{array}{l}\text { REDOX } \\
\text { Liquor }\end{array}$ & MSG-1 & Batch & 20 & 4 & 11 days & Trace & 10 & (d) \\
\hline $\mathrm{Cs}(\mathrm{I})$ & Groundwater & $\begin{array}{l}\text { Burbank } \\
\text { loamy fine } \\
\text { sand }\end{array}$ & Batch & 8996 & & $16 \mathrm{hrs}$ & Trace & 100 & (e) \\
\hline $\mathrm{Cs}(\mathrm{I})$ & Groundwater & $\begin{array}{l}\text { Burbank } \\
\text { loamy fine } \\
\text { sand }\end{array}$ & Batch & 3538 & & $16 \mathrm{hrs}$ & Trace & 50 & (e) \\
\hline Cs(I) & Groundwater & $\begin{array}{l}\text { Burbank } \\
\text { loamy fine } \\
\text { sand }\end{array}$ & Batch & 1050 & & $16 \mathrm{hrs}$ & Trace & 10 & (e) \\
\hline $\mathrm{Cs}(\mathrm{I})$ & Groundwater & $\begin{array}{l}\text { Burbank } \\
\text { loamy fine } \\
\text { sand }\end{array}$ & Batch & 326 & & $16 \mathrm{hrs}$ & Trace & 5 & (e) \\
\hline $\mathrm{Cs}(\mathrm{I})$ & $3 \mathrm{M} \mathrm{NaNO}_{3}$ & $\begin{array}{l}\text { Burbank } \\
\text { loamy fine } \\
\text { sand }\end{array}$ & Batch & 466 & & $16 \mathrm{hrs}$ & Trace & 100 & (e) \\
\hline $\mathrm{Cs}(\mathrm{I})$ & $3 \mathrm{M} \mathrm{NaNO}_{3}$ & $\begin{array}{l}\text { Burbank } \\
\text { loamy fine } \\
\text { sand }\end{array}$ & Batch & 380 & & $16 \mathrm{hrs}$ & Trace & 50 & (e) \\
\hline $\mathrm{Cs}(\mathrm{I})$ & $3 \mathrm{M} \mathrm{NaNO}_{3}$ & $\begin{array}{l}\text { Burbank } \\
\text { loamy fine } \\
\text { sand }\end{array}$ & Batch & 271 & & $16 \mathrm{hrs}$ & Trace & 10 & (e) \\
\hline $\mathrm{Cs}(\mathrm{I})$ & $3 \mathrm{M} \mathrm{NaNO}_{3}$ & $\begin{array}{l}\text { Burbank } \\
\text { loamy fine } \\
\text { sand }\end{array}$ & Batch & 206 & & $16 \mathrm{hrs}$ & Trace & 5 & (e) \\
\hline $\mathrm{Cs}(\mathrm{I})$ & $0.5 \mathrm{M} \mathrm{NaCl}$ & $\begin{array}{l}\text { Burbank } \\
\text { loamy fine } \\
\text { sand }\end{array}$ & Batch & 1091 & & $16 \mathrm{hrs}$ & Trace & 100 & (e) \\
\hline $\mathrm{Cs}(\mathrm{I})$ & $0.5 \mathrm{M} \mathrm{NaCl}$ & $\begin{array}{l}\text { Burbank } \\
\text { loamy fine } \\
\text { sand }\end{array}$ & Batch & 1445 & & $16 \mathrm{hrs}$ & Trace & 50 & (e) \\
\hline $\mathrm{Cs}(\mathrm{I})$ & $0.5 \mathrm{M} \mathrm{NaCl}$ & $\begin{array}{l}\text { Burbank } \\
\text { loamy fine } \\
\text { sand }\end{array}$ & Batch & 1354 & & $16 \mathrm{hrs}$ & Trace & 10 & (e) \\
\hline $\mathrm{Cs}(\mathrm{I})$ & $0.5 \mathrm{M} \mathrm{NaCl}$ & $\begin{array}{l}\text { Burbank } \\
\text { loamy fine } \\
\text { sand }\end{array}$ & Batch & 1173 & & $16 \mathrm{hrs}$ & Trace & 5 & (e) \\
\hline $\mathrm{Cs}(\mathrm{I})$ & $0.5 \mathrm{M} \mathrm{CaCl}_{2}$ & $\begin{array}{l}\text { Burbank } \\
\text { loamy fine } \\
\text { sand }\end{array}$ & Batch & 5211 & & $16 \mathrm{hrs}$ & Trace & 100 & (e) \\
\hline
\end{tabular}




\begin{tabular}{|c|c|c|c|c|c|c|c|c|c|}
\hline Species & $\begin{array}{l}\text { Aqueous } \\
\text { Phase }\end{array}$ & Solid Phase & Method & $\begin{array}{c}\mathrm{Kd} \\
(\mathrm{mL} / \mathrm{g})\end{array}$ & Std. Dev. & $\begin{array}{l}\text { Equil. } \\
\text { Time }\end{array}$ & $\begin{array}{l}\text { In. Soln. } \\
\text { Conc. }\end{array}$ & $\begin{array}{l}\text { Solution/ } \\
\text { Solid } \\
(\mathrm{mL} / \mathrm{g})\end{array}$ & Reference \\
\hline $\mathrm{Cs}(\mathrm{I})$ & $0.5 \mathrm{M} \mathrm{CaCl}_{2}$ & $\begin{array}{l}\text { Burbank } \\
\text { loamy fine } \\
\text { sand }\end{array}$ & Batch & 5362 & & $16 \mathrm{hrs}$ & Trace & 50 & (e) \\
\hline $\mathrm{Cs}(\mathrm{I})$ & $0.5 \mathrm{M} \mathrm{CaCl}_{2}$ & $\begin{array}{l}\text { Burbank } \\
\text { loamy fine } \\
\text { sand }\end{array}$ & Batch & 2420 & & $16 \mathrm{hrs}$ & Trace & 10 & (e) \\
\hline $\mathrm{Cs}(\mathrm{I})$ & $0.5 \mathrm{M} \mathrm{CaCl}_{2}$ & $\begin{array}{l}\text { Burbank } \\
\text { loamy fine } \\
\text { sand }\end{array}$ & Batch & 1264 & & $16 \mathrm{hrs}$ & Trace & 5 & (e) \\
\hline $\mathrm{Cs}(\mathrm{I})$ & $0.2 \mathrm{M} \mathrm{NaCl}$ & $\begin{array}{l}\text { Ritzville } \\
\text { A12 }\end{array}$ & Batch & 4869 & & 1-2 wks* & Trace* & $20 *$ & (f) \\
\hline $\mathrm{Cs}(\mathrm{I})$ & $0.2 \mathrm{M} \mathrm{NaCl}$ & Ritzville B1 & Batch & 4332 & & $1-2 \mathrm{wks}^{*}$ & Trace* & $20 *$ & (f) \\
\hline $\mathrm{Cs}(\mathrm{I})$ & $0.2 \mathrm{M} \mathrm{NaCl}$ & Ritzville C & Batch & 3111 & & $1-2 \mathrm{wks}^{*}$ & Trace* & $20 *$ & (f) \\
\hline $\mathrm{Cs}(\mathrm{I})$ & $0.2 \mathrm{M} \mathrm{NaCl}$ & Ritzville Cca & Batch & 3529 & & 1-2 wks* & Trace* & $20 *$ & (f) \\
\hline $\mathrm{Cs}(\mathrm{I})$ & $0.2 \mathrm{M} \mathrm{NaCl}$ & $\begin{array}{l}\text { Burbank } \\
\text { A12 }\end{array}$ & Batch & 2775 & & $1-2$ wks* & Trace* & $20 *$ & (f) \\
\hline $\mathrm{Cs}(\mathrm{I})$ & $0.2 \mathrm{M} \mathrm{NaCl}$ & Burbank AC & Batch & 2680 & & 1-2 wks* & Trace* & $20 *$ & (f) \\
\hline $\mathrm{Cs}(\mathrm{I})$ & $0.2 \mathrm{M} \mathrm{NaCl}$ & $\begin{array}{l}\text { Burbank } \\
\text { AC2 }\end{array}$ & Batch & 2573 & & $1-2$ wks* & Trace* & $20 *$ & (f) \\
\hline $\mathrm{Cs}(\mathrm{I})$ & $0.2 \mathrm{M} \mathrm{NaCl}$ & Burbank IC & Batch & 1588 & & 1-2 wks* & Trace* & $20 *$ & (f) \\
\hline $\mathrm{Cs}(\mathrm{I})$ & $0.2 \mathrm{M} \mathrm{NaCl}$ & Ephrata A12 & Batch & 3941 & & $1-2$ wks* & Trace* & $20 *$ & (f) \\
\hline $\mathrm{Cs}(\mathrm{I})$ & $0.2 \mathrm{M} \mathrm{NaCl}$ & Ephrata B1 & Batch & 4696 & & $1-2 \mathrm{wks}^{*}$ & Trace* & $20 *$ & (f) \\
\hline $\mathrm{Cs}(\mathrm{I})$ & $0.2 \mathrm{M} \mathrm{NaCl}$ & Ephrata IB2 & Batch & 3491 & & 1-2 wks* & Trace* & $20 *$ & (f) \\
\hline $\mathrm{Cs}(\mathrm{I})$ & $0.2 \mathrm{M} \mathrm{NaCl}$ & Ephrata IIC & Batch & 1919 & & 1-2 wks* & Trace* & $20 *$ & (f) \\
\hline $\mathrm{Cs}(\mathrm{I})$ & T-106 Soln. 1 & Sed 1 & Batch & 13.5 & & 1-2 wks* & Trace* & $20 *$ & (g) \\
\hline $\mathrm{Cs}(\mathrm{I})$ & T-106 Soln. 2 & Sed 1 & Batch & 6 & & $1-2 \mathrm{wks}^{*}$ & Trace* & $20 *$ & (g) \\
\hline $\mathrm{Cs}(\mathrm{I})$ & T-106 Soln. 3 & Sed 1 & Batch & 1.8 & & 1-2 wks* & Trace* & $20 *$ & (g) \\
\hline $\mathrm{Cs}(\mathrm{I})$ & T-106 Soln. 4 & Sed 1 & Batch & 0.8 & & 1-2 wks* & Trace* & $20 *$ & (g) \\
\hline $\mathrm{Cs}(\mathrm{I})$ & T-106 Soln. 5 & Sed 1 & Batch & 12 & & 1-2 wks* & Trace* & $20 *$ & (g) \\
\hline $\mathrm{Cs}(\mathrm{I})$ & T-106 Soln. 1 & Sed 2 & Batch & 9.4 & & 1-2 wks* & Trace* & $20 *$ & (g) \\
\hline $\mathrm{Cs}(\mathrm{I})$ & T-106 Soln. 2 & Sed 2 & Batch & 3.5 & & $1-2$ wks* & Trace* & $20 *$ & (g) \\
\hline $\mathrm{Cs}(\mathrm{I})$ & T-106 Soln. 3 & Sed 2 & Batch & 1.4 & & 1-2 wks* & Trace* & $20 *$ & (g) \\
\hline $\mathrm{Cs}(\mathrm{I})$ & T-106 Soln. 4 & Sed 2 & Batch & 0.6 & & 1-2 wks* & Trace* & $20 *$ & (g) \\
\hline $\mathrm{Cs}(\mathrm{I})$ & T-106 Soln. 5 & Sed 2 & Batch & 7.1 & & $1-2$ wks* & Trace* & $20 *$ & (g) \\
\hline $\mathrm{Cs}(\mathrm{I})$ & T-106 Soln. 1 & Sed 3 & Batch & 9.1 & & 1-2 wks* & Trace* & $20 *$ & (g) \\
\hline $\mathrm{Cs}(\mathrm{I})$ & T-106 Soln. 2 & Sed 3 & Batch & 3.4 & & $1-2 \mathrm{wks}^{*}$ & Trace* & $20 *$ & (g) \\
\hline
\end{tabular}




\begin{tabular}{|c|c|c|c|c|c|c|c|c|c|}
\hline Species & $\begin{array}{c}\text { Aqueous } \\
\text { Phase }\end{array}$ & Solid Phase & Method & $\begin{array}{c}\mathrm{Kd} \\
(\mathrm{mL} / \mathrm{g})\end{array}$ & Std. Dev. & $\begin{array}{c}\text { Equil. } \\
\text { Time }\end{array}$ & $\begin{array}{l}\text { In. Soln. } \\
\text { Conc. }\end{array}$ & $\begin{array}{l}\text { Solution/ } \\
\text { Solid } \\
(\mathrm{mL} / \mathrm{g})\end{array}$ & Reference \\
\hline $\mathrm{Cs}(\mathrm{I})$ & T-106 Soln. 3 & Sed 3 & Batch & 1.3 & & $1-2 w k s^{*}$ & Trace* & $20 *$ & (g) \\
\hline $\mathrm{Cs}(\mathrm{I})$ & T-106 Soln. 4 & Sed 3 & Batch & 0.6 & & $1-2 \mathrm{wks}^{*}$ & Trace* & $20 *$ & (g) \\
\hline $\mathrm{Cs}(\mathrm{I})$ & T-106 Soln. 5 & Sed 3 & Batch & 7.2 & & $1-2 \mathrm{wks}^{*}$ & Trace* & $20 *$ & (g) \\
\hline $\mathrm{Cs}(\mathrm{I})$ & T-106 Soln. 1 & Sed 4 & Batch & 8.1 & & $1-2$ wks* & Trace* & $20 *$ & (g) \\
\hline $\mathrm{Cs}(\mathrm{I})$ & T-106 Soln. 2 & Sed 4 & Batch & 3.1 & & $1-2 \mathrm{wks}^{*}$ & Trace* & $20 *$ & (g) \\
\hline $\mathrm{Cs}(\mathrm{I})$ & T-106 Soln. 3 & Sed 4 & Batch & 1.4 & & $1-2 \mathrm{wks}^{*}$ & Trace* & $20 *$ & (g) \\
\hline $\mathrm{Cs}(\mathrm{I})$ & T-106 Soln. 4 & Sed 4 & Batch & 0.7 & & $1-2$ wks* $^{*}$ & Trace* & $20 *$ & (g) \\
\hline $\mathrm{Cs}(\mathrm{I})$ & T-106 Soln. 5 & Sed 4 & Batch & 7.7 & & $1-2 \mathrm{wks}^{*}$ & Trace* & $20 *$ & (g) \\
\hline $\mathrm{Cs}(\mathrm{I})$ & T-106 Soln. 1 & Sed 5 & Batch & 10.9 & & $1-2$ wks* & Trace* & $20 *$ & (g) \\
\hline $\mathrm{Cs}(\mathrm{I})$ & T-106 Soln. 2 & Sed 5 & Batch & 3.7 & & $1-2$ wks* & Trace* & $20 *$ & (g) \\
\hline $\mathrm{Cs}(\mathrm{I})$ & T-106 Soln. 3 & Sed 5 & Batch & 1.8 & & $1-2$ wks* & Trace* & $20 *$ & (g) \\
\hline $\mathrm{Cs}(\mathrm{I})$ & T-106 Soln. 4 & Sed 5 & Batch & 2.9 & & $1-2 \mathrm{wks}^{*}$ & Trace* & $20 *$ & (g) \\
\hline $\mathrm{Cs}(\mathrm{I})$ & T-106 Soln. 5 & Sed 5 & Batch & 11.3 & & $1-2 \mathrm{wks}^{*}$ & Trace* & $20 *$ & (g) \\
\hline $\mathrm{Cs}(\mathrm{I})$ & T-106 Soln. 1 & Sed 6 & Batch & 7.9 & & $1-2 \mathrm{wks}^{*}$ & Trace* & $20 *$ & (g) \\
\hline $\mathrm{Cs}(\mathrm{I})$ & T-106 Soln. 2 & Sed 6 & Batch & 2 & & $1-2$ wks* & Trace* & $20 *$ & (g) \\
\hline $\mathrm{Cs}(\mathrm{I})$ & T-106 Soln. 3 & Sed 6 & Batch & 0.9 & & $1-2$ wks* $^{*}$ & Trace* & $20 *$ & (g) \\
\hline $\mathrm{Cs}(\mathrm{I})$ & T-106 Soln. 4 & Sed 6 & Batch & 1.2 & & $1-2$ wks* & Trace* & $20 *$ & (g) \\
\hline $\mathrm{Cs}(\mathrm{I})$ & T-106 Soln. 5 & Sed 6 & Batch & 7.9 & & $1-2 \mathrm{wks}^{*}$ & Trace* & $20 *$ & (g) \\
\hline $\mathrm{Cs}(\mathrm{I})$ & T-106 Soln. 1 & Sed 7 & Batch & 8.7 & & $1-2$ wks* & Trace* & $20 *$ & (g) \\
\hline $\mathrm{Cs}(\mathrm{I})$ & T-106 Soln. 2 & Sed 7 & Batch & 2.8 & & $1-2 \mathrm{wks}^{*}$ & Trace* & $20 *$ & (g) \\
\hline $\mathrm{Cs}(\mathrm{I})$ & T-106 Soln. 3 & Sed 7 & Batch & 1.1 & & $1-2 \mathrm{wks}^{*}$ & Trace* & $20 *$ & (g) \\
\hline $\mathrm{Cs}(\mathrm{I})$ & T-106 Soln. 4 & Sed 7 & Batch & 1.5 & & $1-2 \mathrm{wks}^{*}$ & Trace* & $20 *$ & (g) \\
\hline $\mathrm{Cs}(\mathrm{I})$ & T-106 Soln. 5 & Sed 7 & Batch & 9.5 & & $1-2$ wks* & Trace* & $20 *$ & (g) \\
\hline \multicolumn{10}{|c|}{$\begin{array}{l}\text { (a) Serne et al. 1993. } \\
\text { (b) Gee and Campbell } 1980 . \\
\text { (c) Kaplan et al. 1998a. } \\
\text { (d) Serne et al. 1998. } \\
\text { (e) Hajek and Ames } 1966 . \\
\text { (f) } \text { Routson 1973. } \\
\text { (g) Serne et al. 1998. } \\
\text { *Data is best estimate. }\end{array}$} \\
\hline
\end{tabular}


Table 6. Contaminant Distribution Coefficient Data for Nickel

\begin{tabular}{|c|c|c|c|c|c|c|c|c|c|}
\hline Species & $\begin{array}{c}\text { Aqueous } \\
\text { Phase }\end{array}$ & Solid Phase & Method & $\begin{array}{c}\mathrm{Kd} \\
(\mathrm{mL} / \mathrm{g})\end{array}$ & Std. Dev. & $\begin{array}{l}\text { Equil. } \\
\text { Time }\end{array}$ & $\begin{array}{l}\text { In. Soln. } \\
\text { Conc. }\end{array}$ & $\begin{array}{l}\text { Solution/ } \\
\text { Solid } \\
(\mathrm{mL} / \mathrm{g})\end{array}$ & Reference \\
\hline $\mathrm{Ni}(\mathrm{II})$ & Hanford GW & Trench-8 & Batch & $4.40 \mathrm{E}+02$ & $3.00 \mathrm{E}+01$ & 5 days & Trace & 30 & (a) \\
\hline $\mathrm{Ni}(\mathrm{II})$ & Hanford GW & Trench-8 & Batch & $2.35 \mathrm{E}+03$ & $6.80 \mathrm{E}+02$ & 44 days & Trace & 30 & (a) \\
\hline $\mathrm{Ni}(\mathrm{II})$ & Hanford GW3 & 218-E-12B & Batch & 180.0 & & 7 days & $5.0 \mathrm{E}-5 \mathrm{M}$ & 40 & $(b, c)$ \\
\hline $\mathrm{Ni}(\mathrm{II})$ & Hanford GW3 & 218-E-12B & Batch & 155.0 & & 7 days & 5.0E-5 M & 40 & $(b, c)$ \\
\hline $\mathrm{Ni}(\mathrm{II})$ & Hanford GW3 & 218-E-12B & Batch & 155.0 & & 7 days & $5.0 \mathrm{E}-5 \mathrm{M}$ & 40 & $(b, c)$ \\
\hline $\mathrm{Ni}(\mathrm{II})$ & Hanford GW3 & 218-E-12B & Batch & 126.0 & & 7 days & $5.0 \mathrm{E}-5 \mathrm{M}$ & 40 & $(b, c)$ \\
\hline $\mathrm{Ni}(\mathrm{II})$ & Hanford GW3 & 218-E-12B & Batch & 171.0 & & 7 days & $5.0 \mathrm{E}-5 \mathrm{M}$ & 40 & $(b, c)$ \\
\hline $\mathrm{Ni}(\mathrm{II})$ & Hanford GW3 & 218-E-12B & Batch & 154.0 & & 7 days & $5.0 \mathrm{E}-5 \mathrm{M}$ & 40 & $(b, c)$ \\
\hline $\mathrm{Ni}(\mathrm{II})$ & Hanford GW3 & 218-E-12B & Batch & 251.0 & & 7 days & $5.0 \mathrm{E}-5 \mathrm{M}$ & 40 & $(b, c)$ \\
\hline $\mathrm{Ni}(\mathrm{II})$ & Hanford GW3 & 218-E-12B & Batch & 165.0 & & 7 days & $5.0 \mathrm{E}-5 \mathrm{M}$ & 40 & $(b, c)$ \\
\hline $\mathrm{Ni}(\mathrm{II})$ & Hanford GW3 & 218-E-12B & Batch & 189.0 & & 7 days & $5.0 \mathrm{E}-5 \mathrm{M}$ & 40 & $(b, c)$ \\
\hline $\mathrm{Ni}(\mathrm{II})$ & Hanford GW3 & 218-E-12B & Batch & 194.0 & & 7 days & $5.0 \mathrm{E}-5 \mathrm{M}$ & 40 & $(b, c)$ \\
\hline $\mathrm{Ni}(\mathrm{II})$ & Hanford GW3 & 218-E-12B & Batch & 187.0 & & 7 days & $5.0 \mathrm{E}-5 \mathrm{M}$ & 40 & $(b, c)$ \\
\hline $\mathrm{Ni}(\mathrm{II})$ & Hanford GW3 & 218-E-12B & Batch & 192.0 & & 7 days & $5.0 \mathrm{E}-5 \mathrm{M}$ & 40 & $(b, c)$ \\
\hline $\mathrm{Ni}(\mathrm{II})$ & Hanford GW3 & 218-E-12B & Batch & 117.0 & & 7 days & $1.0 \mathrm{E}-5 \mathrm{M}$ & 40 & $(b, c)$ \\
\hline $\mathrm{Ni}(\mathrm{II})$ & Hanford GW3 & 218-E-12B & Batch & 126.0 & & 7 days & $1.0 \mathrm{E}-5 \mathrm{M}$ & 40 & $(b, c)$ \\
\hline $\mathrm{Ni}(\mathrm{II})$ & Hanford GW3 & 218-E-12B & Batch & 116.0 & & 7 days & $1.0 \mathrm{E}-5 \mathrm{M}$ & 40 & $(b, c)$ \\
\hline $\mathrm{Ni}(\mathrm{II})$ & Hanford GW3 & 218-E-12B & Batch & 128.0 & & 7 days & $1.0 \mathrm{E}-5 \mathrm{M}$ & 40 & $(b, c)$ \\
\hline $\mathrm{Ni}(\mathrm{II})$ & Hanford GW3 & 218-E-12B & Batch & 169.0 & & 7 days & 2.0E-6 M & 40 & $(b, c)$ \\
\hline $\mathrm{Ni}(\mathrm{II})$ & Hanford GW3 & 218-E-12B & Batch & 163.0 & & 7 days & 2.0E-6 M & 40 & $(b, c)$ \\
\hline $\mathrm{Ni}(\mathrm{II})$ & Hanford GW3 & 218-E-12B & Batch & 130.0 & & 7 days & 4.0E-7 M & 40 & $(b, c)$ \\
\hline $\mathrm{Ni}(\mathrm{II})$ & Hanford GW3 & 218-E-12B & Batch & 118.0 & & 7 days & 4.0E-7 M & 40 & $(b, c)$ \\
\hline $\mathrm{Ni}(\mathrm{II})$ & Hanford GW3 & 218-E-12B & Batch & 61.4 & & 10 days & $5.0 \mathrm{E}-5 \mathrm{M}$ & 40 & $(b, c)$ \\
\hline $\mathrm{Ni}(\mathrm{II})$ & Hanford GW3 & 218-E-12B & Batch & 48.0 & & 10 days & $5.0 \mathrm{E}-5 \mathrm{M}$ & 40 & $(b, c)$ \\
\hline $\mathrm{Ni}(\mathrm{II})$ & Hanford GW3 & 218-E-12B & Batch & 83.5 & & 10 days & $1.0 \mathrm{E}-5 \mathrm{M}$ & 40 & $(b, c)$ \\
\hline $\mathrm{Ni}(\mathrm{II})$ & Hanford GW3 & 218-E-12B & Batch & 94.3 & & 10 days & $1.0 \mathrm{E}-5 \mathrm{M}$ & 40 & $(b, c)$ \\
\hline $\mathrm{Ni}(\mathrm{II})$ & Hanford GW3 & 218-E-12B & Batch & 212.0 & & 10 days & $2.0 \mathrm{E}-6 \mathrm{M}$ & 40 & $(b, c)$ \\
\hline $\mathrm{Ni}(\mathrm{II})$ & Hanford GW3 & 218-E-12B & Batch & 195.0 & & 10 days & 2.0E-6 M & 40 & $(b, c)$ \\
\hline $\mathrm{Ni}(\mathrm{II})$ & Hanford GW3 & 218-E-12B & Batch & 337.0 & & 10 days & $4.0 \mathrm{E}-7 \mathrm{M}$ & 40 & $(b, c)$ \\
\hline $\mathrm{Ni}(\mathrm{II})$ & Hanford GW3 & 218-E-12B & Batch & 255.0 & & 10 days & $4.0 \mathrm{E}-7 \mathrm{M}$ & 40 & $(b, c)$ \\
\hline $\mathrm{Ni}(\mathrm{II})$ & Hanford GW3 & 218-E-12B & Batch & 54.3 & & 10 days & 5.0E-5 M & 40 & $(b, c)$ \\
\hline $\mathrm{Ni}(\mathrm{II})$ & Hanford GW3 & 218-E-12B & Batch & 63.1 & & 10 days & $5.0 \mathrm{E}-5 \mathrm{M}$ & 40 & $(b, c)$ \\
\hline $\mathrm{Ni}(\mathrm{II})$ & Hanford GW3 & 218-E-12B & Batch & 52.5 & & 10 days & $5.0 \mathrm{E}-5 \mathrm{M}$ & 40 & $(b, c)$ \\
\hline $\mathrm{Ni}(\mathrm{II})$ & Hanford GW3 & 218-E-12B & Batch & 74.0 & & 10 days & $1.0 \mathrm{E}-5 \mathrm{M}$ & 40 & $(b, c)$ \\
\hline
\end{tabular}




\begin{tabular}{|c|c|c|c|c|c|c|c|c|c|}
\hline Species & $\begin{array}{l}\text { Aqueous } \\
\text { Phase }\end{array}$ & Solid Phase & Method & $\begin{array}{c}\mathrm{Kd} \\
(\mathrm{mL} / \mathrm{g})\end{array}$ & Std. Dev. & $\begin{array}{l}\text { Equil. } \\
\text { Time }\end{array}$ & $\begin{array}{l}\text { In. Soln. } \\
\text { Conc. }\end{array}$ & $\begin{array}{l}\text { Solution/ } \\
\text { Solid } \\
(\mathrm{mL} / \mathrm{g})\end{array}$ & Reference \\
\hline $\mathrm{Ni}(\mathrm{II})$ & Hanford GW3 & 218-E-12B & Batch & 95.9 & & 10 days & $1.0 \mathrm{E}-5 \mathrm{M}$ & 40 & $(b, c)$ \\
\hline $\mathrm{Ni}(\mathrm{II})$ & Hanford GW3 & 218-E-12B & Batch & 87.0 & & 10 days & $1.0 \mathrm{E}-5 \mathrm{M}$ & 40 & $(b, c)$ \\
\hline $\mathrm{Ni}(\mathrm{II})$ & Hanford GW3 & 218-E-12B & Batch & 169.0 & & 10 days & $2.0 \mathrm{E}-6 \mathrm{M}$ & 40 & $(b, c)$ \\
\hline $\mathrm{Ni}(\mathrm{II})$ & Hanford GW3 & 218-E-12B & Batch & 143.0 & & 10 days & $2.0 \mathrm{E}-6 \mathrm{M}$ & 40 & $(b, c)$ \\
\hline $\mathrm{Ni}(\mathrm{II})$ & Hanford GW3 & 218-E-12B & Batch & 168.0 & & 10 days & $2.0 \mathrm{E}-6 \mathrm{M}$ & 40 & $(b, c)$ \\
\hline $\mathrm{Ni}(\mathrm{II})$ & Hanford GW3 & 218-E-12B & Batch & 311.0 & & 10 days & 4.0E-7 M & 40 & $(b, c)$ \\
\hline $\mathrm{Ni}(\mathrm{II})$ & Hanford GW3 & 218-E-12B & Batch & 259.0 & & 10 days & $4.0 \mathrm{E}-7 \mathrm{M}$ & 40 & $(b, c)$ \\
\hline $\mathrm{Ni}(\mathrm{II})$ & Hanford GW3 & 218-E-12B & Batch & 247.0 & & 10 days & $4.0 \mathrm{E}-7 \mathrm{M}$ & 40 & $(b, c)$ \\
\hline $\mathrm{Ni}(\mathrm{II})$ & Hanford GW3 & 218-E-12B & Batch & 179.0 & & 26 days & $5.0 \mathrm{E}-5 \mathrm{M}$ & 40 & $(b, c)$ \\
\hline $\mathrm{Ni}(\mathrm{II})$ & Hanford GW3 & 218-E-12B & Batch & 184.0 & & 26 days & $5.0 \mathrm{E}-5 \mathrm{M}$ & 40 & $(b, c)$ \\
\hline $\mathrm{Ni}(\mathrm{II})$ & Hanford GW3 & 218-E-12B & Batch & 135.0 & & 26 days & $1.0 \mathrm{E}-5 \mathrm{M}$ & 40 & $(b, c)$ \\
\hline $\mathrm{Ni}(\mathrm{II})$ & Hanford GW3 & 218-E-12B & Batch & 170.0 & & 26 days & $1.0 \mathrm{E}-5 \mathrm{M}$ & 40 & $(b, c)$ \\
\hline $\mathrm{Ni}(\mathrm{II})$ & Hanford GW3 & 218-E-12B & Batch & 368.0 & & 26 days & $2.0 \mathrm{E}-6 \mathrm{M}$ & 40 & $(b, c)$ \\
\hline $\mathrm{Ni}(\mathrm{II})$ & Hanford GW3 & 218-E-12B & Batch & 264.0 & & 26 days & $2.0 \mathrm{E}-6 \mathrm{M}$ & 40 & $(b, c)$ \\
\hline $\mathrm{Ni}(\mathrm{II})$ & Hanford GW3 & 218-E-12B & Batch & 421.0 & & 26 days & $4.0 \mathrm{E}-7 \mathrm{M}$ & 40 & $(b, c)$ \\
\hline $\mathrm{Ni}(\mathrm{II})$ & Hanford GW3 & 218-E-12B & Batch & 592.0 & & 26 days & $4.0 \mathrm{E}-7 \mathrm{M}$ & 40 & $(b, c)$ \\
\hline \multicolumn{10}{|c|}{\begin{tabular}{|ll} 
(a) & Serne et al. 1993. \\
(b) & Rhoades et al. 1992. \\
(c) & Rhoades et al. 1994. \\
\end{tabular}} \\
\hline
\end{tabular}


Table 7. Contaminant Distribution Coefficient Data for Lead

\begin{tabular}{|c|c|c|c|c|c|c|c|c|c|}
\hline Species & $\begin{array}{c}\text { Aqueous } \\
\text { Phase }\end{array}$ & Solid Phase & Method & $\begin{array}{c}\mathrm{Kd} \\
(\mathrm{mL} / \mathrm{g})\end{array}$ & Std. Dev. & $\begin{array}{l}\text { Equil. } \\
\text { Time }\end{array}$ & $\begin{array}{c}\text { In. Soln. } \\
\text { Conc. }\end{array}$ & $\begin{array}{l}\text { Solution/ } \\
\text { Solid } \\
(\mathrm{mL} / \mathrm{g})\end{array}$ & Reference \\
\hline $\mathrm{Pb}(\mathrm{II})$ & Hanford GW3 & 218-E-12B & Batch & 8230.0 & & 7 days & 1.0E-6 M & 40 & $(a, b)$ \\
\hline $\mathrm{Pb}(\mathrm{II})$ & Hanford GW3 & 218-E-12B & Batch & 13700.0 & & 7 days & $1.0 \mathrm{E}-6 \mathrm{M}$ & 40 & $(a, b)$ \\
\hline $\mathrm{Pb}(\mathrm{II})$ & Hanford GW3 & 218-E-12B & Batch & 4050.0 & & 7 days & $1.0 \mathrm{E}-7 \mathrm{M}$ & 40 & $(a, b)$ \\
\hline $\mathrm{Pb}(\mathrm{II})$ & Hanford GW3 & 218-E-12B & Batch & 4140.0 & & 7 days & $1.0 \mathrm{E}-7 \mathrm{M}$ & 40 & $(a, b)$ \\
\hline $\mathrm{Pb}(\mathrm{II})$ & Hanford GW3 & 218-E-12B & Batch & 8510.0 & & 7 days & $1.0 \mathrm{E}-6 \mathrm{M}$ & 40 & $(a, b)$ \\
\hline $\mathrm{Pb}(\mathrm{II})$ & Hanford GW3 & 218-E-12B & Batch & 5060.0 & & 7 days & $1.0 \mathrm{E}-6 \mathrm{M}$ & 40 & $(a, b)$ \\
\hline $\mathrm{Pb}(\mathrm{II})$ & Hanford GW3 & 218-E-12B & Batch & 6160.0 & & 7 days & 3.0E-7 M & 40 & $(a, b)$ \\
\hline $\mathrm{Pb}(\mathrm{II})$ & Hanford GW3 & 218-E-12B & Batch & 8140.0 & & 7 days & 3.0E-7 M & 40 & $(a, b)$ \\
\hline $\mathrm{Pb}(\mathrm{II})$ & Hanford GW3 & 218-E-12B & Batch & 4720.0 & & 7 days & $1.0 \mathrm{E}-7 \mathrm{M}$ & 40 & $(a, b)$ \\
\hline $\mathrm{Pb}(\mathrm{II})$ & Hanford GW3 & 218-E-12B & Batch & 5140.0 & & 7 days & $1.0 \mathrm{E}-7 \mathrm{M}$ & 40 & $(a, b)$ \\
\hline $\mathrm{Pb}(\mathrm{II})$ & Hanford GW3 & 218-E-12B & Batch & 1330.0 & & 7 days & $3.0 \mathrm{E}-8 \mathrm{M}$ & 40 & $(a, b)$ \\
\hline $\mathrm{Pb}(\mathrm{II})$ & Hanford GW3 & 218-E-12B & Batch & 1670.0 & & 7 days & 3.0E-8 M & 40 & $(a, b)$ \\
\hline $\mathrm{Pb}(\mathrm{II})$ & Hanford GW3 & 218-E-12B & Batch & 5080.0 & & 7 days & 1.0E-6 M & 40 & $(a, b)$ \\
\hline $\mathrm{Pb}(\mathrm{II})$ & Hanford GW3 & 218-E-12B & Batch & 4990.0 & & 7 days & $1.0 \mathrm{E}-6 \mathrm{M}$ & 40 & $(a, b)$ \\
\hline $\mathrm{Pb}(\mathrm{II})$ & Hanford GW3 & 218-E-12B & Batch & 5530.0 & & 7 days & $1.0 \mathrm{E}-6 \mathrm{M}$ & 40 & $(a, b)$ \\
\hline $\mathrm{Pb}(\mathrm{II})$ & Hanford GW3 & 218-E-12B & Batch & 6070.0 & & 7 days & 1.0E-6 M & 40 & $(a, b)$ \\
\hline $\mathrm{Pb}(\mathrm{II})$ & Hanford GW3 & 218-E-12B & Batch & 9000.0 & & 7 days & 1.0E-6 M & 40 & $(a, b)$ \\
\hline $\mathrm{Pb}(\mathrm{II})$ & Hanford GW3 & 218-E-12B & Batch & 8730.0 & & 7 days & 1.0E-6 M & 40 & $(a, b)$ \\
\hline $\mathrm{Pb}(\mathrm{II})$ & Hanford GW3 & 218-E-12B & Batch & 8130.0 & & 7 days & $1.0 \mathrm{E}-6 \mathrm{M}$ & 40 & $(a, b)$ \\
\hline $\mathrm{Pb}(\mathrm{II})$ & Hanford GW3 & 218-E-12B & Batch & 4520.0 & & 7 days & $1.0 \mathrm{E}-6 \mathrm{M}$ & 40 & $(a, b)$ \\
\hline $\mathrm{Pb}(\mathrm{II})$ & Hanford GW3 & 218-E-12B & Batch & 29200.0 & & 10 days & 1.0E-6 M & 40 & $(a, b)$ \\
\hline $\mathrm{Pb}(\mathrm{II})$ & Hanford GW3 & 218-E-12B & Batch & 24800.0 & & 10 days & 1.0E-6 M & 40 & $(a, b)$ \\
\hline $\mathrm{Pb}(\mathrm{II})$ & Hanford GW3 & 218-E-12B & Batch & 16000.0 & & 10 days & 3.0E-7 M & 40 & $(a, b)$ \\
\hline $\mathrm{Pb}(\mathrm{II})$ & Hanford GW3 & 218-E-12B & Batch & 32500.0 & & 10 days & $3.0 \mathrm{E}-7 \mathrm{M}$ & 40 & $(a, b)$ \\
\hline $\mathrm{Pb}(\mathrm{II})$ & Hanford GW3 & 218-E-12B & Batch & 78700.0 & & 10 days & $1.0 \mathrm{E}-7 \mathrm{M}$ & 40 & $(a, b)$ \\
\hline $\mathrm{Pb}(\mathrm{II})$ & Hanford GW3 & 218-E-12B & Batch & 51000.0 & & 10 days & $1.0 \mathrm{E}-7 \mathrm{M}$ & 40 & $(a, b)$ \\
\hline $\mathrm{Pb}(\mathrm{II})$ & Hanford GW3 & 218-E-12B & Batch & 30400.0 & & 10 days & $3.0 \mathrm{E}-8 \mathrm{M}$ & 40 & $(a, b)$ \\
\hline $\mathrm{Pb}(\mathrm{II})$ & Hanford GW3 & 218-E-12B & Batch & 41400.0 & & 10 days & $3.0 \mathrm{E}-8 \mathrm{M}$ & 40 & $(a, b)$ \\
\hline $\mathrm{Pb}(\mathrm{II})$ & Hanford GW3 & 218-E-12B & Batch & 19200.0 & & 10 days & $1.0 \mathrm{E}-6 \mathrm{M}$ & 40 & $(a, b)$ \\
\hline $\mathrm{Pb}$ (II) & Hanford GW3 & 218-E-12B & Batch & 18000.0 & & 10 days & 1.0E-6 M & 40 & $(a, b)$ \\
\hline $\mathrm{Pb}(\mathrm{II})$ & Hanford GW3 & 218-E-12B & Batch & 12500.0 & & 10 days & $1.0 \mathrm{E}-6 \mathrm{M}$ & 40 & $(a, b)$ \\
\hline $\mathrm{Pb}$ (II) & Hanford GW3 & 218-E-12B & Batch & 22400.0 & & 10 days & 3.0E-7 M & 40 & $(a, b)$ \\
\hline $\mathrm{Pb}$ (II) & Hanford GW3 & 218-E-12B & Batch & 30400.0 & & 10 days & 3.0E-7 M & 40 & $(a, b)$ \\
\hline $\mathrm{Pb}(\mathrm{II})$ & Hanford GW3 & 218-E-12B & Batch & 20100.0 & & 10 days & 3.0E-7 M & 40 & $(a, b)$ \\
\hline
\end{tabular}




\begin{tabular}{|c|c|c|c|c|c|c|c|c|c|}
\hline Species & $\begin{array}{l}\text { Aqueous } \\
\text { Phase }\end{array}$ & Solid Phase & Method & $\begin{array}{c}\mathrm{Kd} \\
(\mathrm{mL} / \mathrm{g})\end{array}$ & Std. Dev. & $\begin{array}{l}\text { Equil. } \\
\text { Time }\end{array}$ & $\begin{array}{l}\text { In. Soln. } \\
\text { Conc. }\end{array}$ & $\begin{array}{l}\text { Solution/ } \\
\text { Solid } \\
(\mathrm{mL} / \mathrm{g})\end{array}$ & Reference \\
\hline $\mathrm{Pb}(\mathrm{II})$ & Hanford GW3 & 218-E-12B & Batch & 41000.0 & & 10 days & $1.0 \mathrm{E}-7 \mathrm{M}$ & 40 & $(a, b)$ \\
\hline $\mathrm{Pb}(\mathrm{II})$ & Hanford GW3 & 218-E-12B & Batch & 36500.0 & & 10 days & $1.0 \mathrm{E}-7 \mathrm{M}$ & 40 & $(a, b)$ \\
\hline $\mathrm{Pb}(\mathrm{II})$ & Hanford GW3 & 218-E-12B & Batch & 28700.0 & & 10 days & $1.0 \mathrm{E}-7 \mathrm{M}$ & 40 & $(a, b)$ \\
\hline $\mathrm{Pb}(\mathrm{II})$ & Hanford GW3 & 218-E-12B & Batch & 47900.0 & & 10 days & $3.0 \mathrm{E}-8 \mathrm{M}$ & 40 & $(a, b)$ \\
\hline $\mathrm{Pb}(\mathrm{II})$ & Hanford GW3 & 218-E-12B & Batch & 70800.0 & & 10 days & $3.0 \mathrm{E}-8 \mathrm{M}$ & 40 & $(a, b)$ \\
\hline $\mathrm{Pb}(\mathrm{II})$ & Hanford GW3 & 218-E-12B & Batch & 28100.0 & & 10 days & $3.0 \mathrm{E}-8 \mathrm{M}$ & 40 & $(a, b)$ \\
\hline $\mathrm{Pb}(\mathrm{II})$ & Hanford GW3 & 218-E-12B & Batch & 469000.0 & & 30 days & $1.0 \mathrm{E}-6 \mathrm{M}$ & 40 & $(a, b)$ \\
\hline $\mathrm{Pb}(\mathrm{II})$ & Hanford GW3 & 218-E-12B & Batch & 272000.0 & & 30 days & $1.0 \mathrm{E}-6 \mathrm{M}$ & 40 & $(a, b)$ \\
\hline $\mathrm{Pb}(\mathrm{II})$ & Hanford GW3 & 218-E-12B & Batch & 76500.0 & & 30 days & $1.0 \mathrm{E}-6 \mathrm{M}$ & 40 & $(a, b)$ \\
\hline $\mathrm{Pb}(\mathrm{II})$ & Hanford GW3 & 218-E-12B & Batch & 73600.0 & & 30 days & $1.0 \mathrm{E}-6 \mathrm{M}$ & 40 & $(a, b)$ \\
\hline $\mathrm{Pb}(\mathrm{II})$ & Hanford GW3 & 218-E-12B & Batch & 174000.0 & & 30 days & $1.0 \mathrm{E}-6 \mathrm{M}$ & 40 & $(a, b)$ \\
\hline $\mathrm{Pb}(\mathrm{II})$ & Hanford GW3 & 218-E-12B & Batch & 69800.0 & & 30 days & $1.0 \mathrm{E}-6 \mathrm{M}$ & 40 & $(a, b)$ \\
\hline $\mathrm{Pb}(\mathrm{II})$ & Hanford GW3 & 218-E-12B & Batch & 70700.0 & & 30 days & $1.0 \mathrm{E}-6 \mathrm{M}$ & 40 & $(a, b)$ \\
\hline $\mathrm{Pb}(\mathrm{II})$ & Hanford GW3 & 218-E-12B & Batch & 170000.0 & & 30 days & $1.0 \mathrm{E}-6 \mathrm{M}$ & 40 & $(a, b)$ \\
\hline $\begin{array}{ll}\text { (a) } & R \\
\text { (b) } & R\end{array}$ & les et al. 1992 & & & & & & & & \\
\hline
\end{tabular}


Table 8. Contaminant Distribution Coefficient Data for Plutonium

\begin{tabular}{|c|c|c|c|c|c|c|c|c|c|}
\hline Species & $\begin{array}{c}\text { Aqueous } \\
\text { Phase }\end{array}$ & Solid Phase & Method & $\begin{array}{c}\mathrm{Kd} \\
(\mathrm{mL} / \mathrm{g})\end{array}$ & Std. Dev. & $\begin{array}{c}\text { Equil. } \\
\text { Time }\end{array}$ & $\begin{array}{c}\text { In. Soln. } \\
\text { Conc. }\end{array}$ & $\begin{array}{l}\text { Solution/ } \\
\text { Solid } \\
(\mathrm{mL} / \mathrm{g})\end{array}$ & Reference \\
\hline $\mathrm{Pu}$ & Soln 1 & Sed L & Batch & 30 & & 7 days & Trace & 5 & (a) \\
\hline $\mathrm{Pu}$ & Soln 2 & Sed L & Batch & 5.24 & & 7 days & Trace & 5 & (a) \\
\hline $\mathrm{Pu}$ & Soln 3 & Sed L & Batch & 2.83 & & 7 days & Trace & 5 & (a) \\
\hline $\mathrm{Pu}$ & Soln 4 & Sed L & Batch & 1.27 & & 7 days & Trace & 5 & (a) \\
\hline $\mathrm{Pu}$ & Soln 5 & Sed L & Batch & 2.13 & & 7 days & Trace & 5 & (a) \\
\hline $\mathrm{Pu}$ & Soln 6 & Sed L & Batch & 10.42 & & 7 days & Trace & 5 & (a) \\
\hline $\mathrm{Pu}$ & Soln 7 & Sed L & Batch & 10.15 & & 7 days & Trace & 5 & (a) \\
\hline $\mathrm{Pu}$ & Soln 8 & Sed L & Batch & 3.16 & & 7 days & Trace & 5 & (a) \\
\hline $\mathrm{Pu}$ & Soln 9 & Sed L & Batch & 6.52 & & 7 days & Trace & 5 & (a) \\
\hline $\mathrm{Pu}$ & Soln 10 & Sed L & Batch & 3.61 & & 7 days & Trace & 5 & (a) \\
\hline $\mathrm{Pu}$ & Soln 11 & Sed L & Batch & 42.52 & & 7 days & Trace & 5 & (a) \\
\hline $\mathrm{Pu}$ & Soln 12 & Sed L & Batch & 20.14 & & 7 days & Trace & 5 & (a) \\
\hline $\mathrm{Pu}$ & Soln 13 & Sed L & Batch & 23.54 & & 7 days & Trace & 5 & (a) \\
\hline $\mathrm{Pu}$ & Soln 14 & Sed L & Batch & 94.93 & & 7 days & Trace & 5 & (a) \\
\hline $\mathrm{Pu}$ & Soln 15 & Sed L & Batch & 1.79 & & 7 days & Trace & 5 & (a) \\
\hline $\mathrm{Pu}$ & Soln 16 & Sed L & Batch & 4.03 & & 7 days & Trace & 5 & (a) \\
\hline $\mathrm{Pu}$ & Soln 17 & Sed L & Batch & 4.03 & & 7 days & Trace & 5 & (a) \\
\hline $\mathrm{Pu}$ & Soln 18 & Sed L & Batch & 1.12 & & 7 days & Trace & 5 & (a) \\
\hline $\mathrm{Pu}$ & Soln 19 & Sed L & Batch & 4.21 & & 7 days & Trace & 5 & (a) \\
\hline $\mathrm{Pu}$ & Soln 20 & Sed L & Batch & $>208$ & & 7 days & Trace & 5 & (a) \\
\hline $\mathrm{Pu}$ & Soln 1 & Sed P & Batch & 34.5 & & 7 days & Trace & 5 & (a) \\
\hline $\mathrm{Pu}$ & Soln 2 & Sed P & Batch & 4.82 & & 7 days & Trace & 5 & (a) \\
\hline $\mathrm{Pu}$ & Soln 3 & Sed P & Batch & 2.57 & & 7 days & Trace & 5 & (a) \\
\hline $\mathrm{Pu}$ & Soln 4 & Sed P & Batch & 1.5 & & 7 days & Trace & 5 & (a) \\
\hline $\mathrm{Pu}$ & Soln 5 & Sed P & Batch & 1.98 & & 7 days & Trace & 5 & (a) \\
\hline $\mathrm{Pu}$ & Soln 6 & Sed P & Batch & 7.8 & & 7 days & Trace & 5 & (a) \\
\hline $\mathrm{Pu}$ & Soln 7 & Sed P & Batch & 12.1 & & 7 days & Trace & 5 & (a) \\
\hline $\mathrm{Pu}$ & Soln 8 & Sed P & Batch & 5.68 & & 7 days & Trace & 5 & (a) \\
\hline $\mathrm{Pu}$ & Soln 9 & Sed P & Batch & 5.17 & & 7 days & Trace & 5 & (a) \\
\hline $\mathrm{Pu}$ & Soln 10 & Sed P & Batch & 4.99 & & 7 days & Trace & 5 & (a) \\
\hline $\mathrm{Pu}$ & Soln 11 & Sed P & Batch & 34 & & 7 days & Trace & 5 & (a) \\
\hline $\mathrm{Pu}$ & Soln 12 & Sed P & Batch & 26 & & 7 days & Trace & 5 & (a) \\
\hline $\mathrm{Pu}$ & Soln 13 & Sed P & Batch & 28.5 & & 7 days & Trace & 5 & (a) \\
\hline $\mathrm{Pu}$ & Soln 14 & Sed P & Batch & 124.6 & & 7 days & Trace & 5 & (a) \\
\hline $\mathrm{Pu}$ & Soln 15 & Sed P & Batch & 2.84 & & 7 days & Trace & 5 & (a) \\
\hline $\mathrm{Pu}$ & Soln 16 & Sed P & Batch & 4.86 & & 7 days & Trace & 5 & (a) \\
\hline
\end{tabular}




\begin{tabular}{|c|c|c|c|c|c|c|c|c|c|}
\hline Species & $\begin{array}{l}\text { Aqueous } \\
\text { Phase }\end{array}$ & Solid Phase & Method & $\begin{array}{c}\mathrm{Kd} \\
(\mathrm{mL} / \mathrm{g})\end{array}$ & Std. Dev. & $\begin{array}{c}\text { Equil. } \\
\text { Time }\end{array}$ & $\begin{array}{l}\text { In. Soln. } \\
\text { Conc. }\end{array}$ & $\begin{array}{l}\text { Solution/ } \\
\text { Solid } \\
(\mathrm{mL} / \mathrm{g})\end{array}$ & Reference \\
\hline $\mathrm{Pu}$ & Soln 17 & Sed P & Batch & 4.06 & & 7 days & Trace & 5 & (a) \\
\hline $\mathrm{Pu}$ & Soln 18 & Sed P & Batch & 1.43 & & 7 days & Trace & 5 & (a) \\
\hline $\mathrm{Pu}$ & Soln 19 & Sed P & Batch & 6.53 & & 7 days & Trace & 5 & (a) \\
\hline $\mathrm{Pu}$ & Soln 20 & Sed P & Batch & $>194$ & & 7 days & Trace & 5 & (a) \\
\hline $\mathrm{Pu}$ & Soln 1 & Sed S & Batch & 39 & & 7 days & Trace & 5 & (a) \\
\hline $\mathrm{Pu}$ & Soln 2 & Sed S & Batch & 7.6 & & 7 days & Trace & 5 & (a) \\
\hline $\mathrm{Pu}$ & Soln 3 & Sed S & Batch & 4.46 & & 7 days & Trace & 5 & (a) \\
\hline $\mathrm{Pu}$ & Soln 4 & Sed S & Batch & 5.96 & & 7 days & Trace & 5 & (a) \\
\hline $\mathrm{Pu}$ & Soln 5 & Sed S & Batch & 7.43 & & 7 days & Trace & 5 & (a) \\
\hline $\mathrm{Pu}$ & Soln 6 & Sed S & Batch & 49.3 & & 7 days & \begin{tabular}{|l|} 
Trace \\
\end{tabular} & 5 & (a) \\
\hline $\mathrm{Pu}$ & Soln 7 & Sed S & Batch & 7.15 & & 7 days & Trace & 5 & (a) \\
\hline $\mathrm{Pu}$ & Soln 8 & Sed S & Batch & 11.2 & & 7 days & Trace & 5 & (a) \\
\hline $\mathrm{Pu}$ & Soln 9 & Sed S & Batch & 5.26 & & 7 days & Trace & 5 & (a) \\
\hline $\mathrm{Pu}$ & Soln 10 & Sed S & Batch & 7.92 & & 7 days & Trace & 5 & (a) \\
\hline $\mathrm{Pu}$ & Soln 11 & Sed S & Batch & 44.8 & & 7 days & Trace & 5 & (a) \\
\hline $\mathrm{Pu}$ & Soln 12 & Sed S & Batch & 19.3 & & 7 days & Trace & 5 & (a) \\
\hline $\mathrm{Pu}$ & Soln 13 & Sed S & Batch & 28.7 & & 7 days & Trace & 5 & (a) \\
\hline $\mathrm{Pu}$ & Soln 14 & Sed S & Batch & 145 & & 7 days & Trace & 5 & (a) \\
\hline $\mathrm{Pu}$ & Soln 15 & Sed S & Batch & 5.16 & & 7 days & Trace & 5 & (a) \\
\hline $\mathrm{Pu}$ & Soln 16 & Sed S & Batch & 8.49 & & 7 days & Trace & 5 & (a) \\
\hline $\mathrm{Pu}$ & Soln 17 & Sed S & Batch & 6.62 & & 7 days & Trace & 5 & (a) \\
\hline $\mathrm{Pu}$ & Soln 18 & Sed S & Batch & 2.6 & & 7 days & Trace & 5 & (a) \\
\hline $\mathrm{Pu}$ & Soln 19 & Sed S & Batch & 9.64 & & 7 days & Trace & 5 & (a) \\
\hline $\mathrm{Pu}$ & Soln 20 & Sed S & Batch & $>207$ & & 7 days & Trace & 5 & (a) \\
\hline $\mathrm{Pu}$ & $\begin{array}{l}\text { AAW, pH } \\
1.7^{*}\end{array}$ & 299-W18-6, -7 & Batch & $<1$ & & & & & (b) \\
\hline $\mathrm{Pu}$ & AAW, pH 2 & 299-W18-6, -7 & Batch & $<1$ & & & & & (b) \\
\hline $\mathrm{Pu}$ & AAW, $\mathrm{pH} 3$ & $299-W 18-6,-7$ & Batch & $<1$ & & & & & (b) \\
\hline $\mathrm{Pu}$ & $\begin{array}{l}\text { DI Water } \mathrm{pH} \\
8.0\end{array}$ & 219-S-2 & Batch & $5.20 \mathrm{E}+03$ & & 1 day & 7.70E-07 & 100 & $(\mathrm{c}, \mathrm{d})$ \\
\hline $\mathrm{Pu}$ & $\begin{array}{l}\text { DI Water } \mathrm{pH} \\
8.0\end{array}$ & 219-S-2 & Batch & $4.10 \mathrm{E}+03$ & & 1 day & 7.70E-07 & 100 & $(\mathrm{c}, \mathrm{d})$ \\
\hline $\mathrm{Pu}$ & $\begin{array}{l}\text { DI Water } \mathrm{pH} \\
8.0\end{array}$ & 219-S-2 & Batch & $1.20 \mathrm{E}+03$ & & 1 day & $7.70 \mathrm{E}-07$ & 20 & $(\mathrm{c}, \mathrm{d})$ \\
\hline $\mathrm{Pu}$ & $\begin{array}{l}\text { DI Water } \mathrm{pH} \\
8.0\end{array}$ & 219-S-2 & Batch & $2.50 \mathrm{E}+03$ & & 1 day & 7.70E-07 & 10 & $(\mathrm{c}, \mathrm{d})$ \\
\hline
\end{tabular}




\begin{tabular}{|c|c|c|c|c|c|c|c|c|c|}
\hline Species & $\begin{array}{l}\text { Aqueous } \\
\text { Phase }\end{array}$ & Solid Phase & Method & $\begin{array}{c}\mathrm{Kd} \\
(\mathrm{mL} / \mathrm{g})\end{array}$ & Std. Dev. & $\begin{array}{c}\text { Equil. } \\
\text { Time }\end{array}$ & $\begin{array}{l}\text { In. Soln. } \\
\text { Conc. }\end{array}$ & $\begin{array}{l}\text { Solution/ } \\
\text { Solid } \\
(\mathrm{mL} / \mathrm{g})\end{array}$ & Reference \\
\hline $\mathrm{Pu}$ & $\begin{array}{l}\text { DI Water } \mathrm{pH} \\
8.0\end{array}$ & 219-S-2 & Batch & $1.80 \mathrm{E}+03$ & & 1 day & $1.00 \mathrm{E}-08$ & 20 & $(\mathrm{c}, \mathrm{d})$ \\
\hline $\mathrm{Pu}$ & $\begin{array}{l}\text { DI Water } \mathrm{pH} \\
8.0\end{array}$ & 219-S-2 & Batch & $2.20 \mathrm{E}+03$ & & 1 day & $1.20 \mathrm{E}-07$ & 20 & $(\mathrm{c}, \mathrm{d})$ \\
\hline $\mathrm{Pu}$ & $\begin{array}{l}\text { DI Water } \mathrm{pH} \\
8.0\end{array}$ & 219-S-2 & Batch & $4.00 \mathrm{E}+03$ & & 1 day & $2.10 \mathrm{E}-06$ & 20 & $(\mathrm{c}, \mathrm{d})$ \\
\hline $\mathrm{Pu}$ & $\begin{array}{l}\text { DI Water } \mathrm{pH} \\
8.0\end{array}$ & 219-S-2 & Batch & $4.00 \mathrm{E}+03$ & & 1 day & $3.60 \mathrm{E}-06$ & 20 & $(\mathrm{c}, \mathrm{d})$ \\
\hline $\mathrm{Pu}$ & $\begin{array}{l}\text { DI Water } \mathrm{pH} \\
8.0\end{array}$ & 219-S-2 & Batch & $1.50 \mathrm{E}+03$ & & 1 day & $1.30 \mathrm{E}-05$ & 20 & $(c, d)$ \\
\hline $\mathrm{Pu}$ & $\begin{array}{l}\text { DI Water } \mathrm{pH} \\
8.0\end{array}$ & 219-S-2 & Batch & 506 & & 1 day & $2.10 \mathrm{E}-05$ & 20 & $(\mathrm{c}, \mathrm{d})$ \\
\hline $\mathrm{Pu}$ & $\begin{array}{l}\text { DI Water } \mathrm{pH} \\
0.5\end{array}$ & 219-S-2 & Batch & 28.4 & & 1 day & $7.70 \mathrm{E}-07$ & 20 & $(\mathrm{c}, \mathrm{d})$ \\
\hline $\mathrm{Pu}$ & $\begin{array}{l}\text { DI Water } \mathrm{pH} \\
2.1\end{array}$ & 219-S-2 & Batch & $2.50 \mathrm{E}+03$ & & 1 day & $7.70 \mathrm{E}-07$ & 20 & $(c, d)$ \\
\hline $\mathrm{Pu}$ & $\begin{array}{l}\text { DI Water } \mathrm{pH} \\
3.6\end{array}$ & 219-S-2 & Batch & $2.00 \mathrm{E}+03$ & & 1 day & $7.70 \mathrm{E}-07$ & 20 & $(\mathrm{c}, \mathrm{d})$ \\
\hline $\mathrm{Pu}$ & $\begin{array}{l}\text { DI Water } \mathrm{pH} \\
4.5\end{array}$ & 219-S-2 & Batch & $2.50 \mathrm{E}+03$ & & 1 day & $7.70 \mathrm{E}-07$ & 20 & $(\mathrm{c}, \mathrm{d})$ \\
\hline $\mathrm{Pu}$ & $\begin{array}{l}\text { DI Water } \mathrm{pH} \\
5.3\end{array}$ & 219-S-2 & Batch & $2.50 \mathrm{E}+03$ & & 1 day & 7.70E-07 & 20 & $(\mathrm{c}, \mathrm{d})$ \\
\hline $\mathrm{Pu}$ & $\begin{array}{l}\text { DI Water } \mathrm{pH} \\
6.1\end{array}$ & 219-S-2 & Batch & $1.30 \mathrm{E}+03$ & & 1 day & $7.70 \mathrm{E}-07$ & 20 & $(\mathrm{c}, \mathrm{d})$ \\
\hline $\mathrm{Pu}$ & $\begin{array}{l}\text { DI Water } \mathrm{pH} \\
6.5\end{array}$ & 219-S-2 & Batch & $2.20 \mathrm{E}+03$ & & 1 day & 7.70E-07 & 20 & $(\mathrm{c}, \mathrm{d})$ \\
\hline $\mathrm{Pu}$ & $\begin{array}{l}\text { DI Water } \mathrm{pH} \\
7.3\end{array}$ & 219-S-2 & Batch & $2.00 \mathrm{E}+03$ & & 1 day & 7.70E-07 & 20 & $(c, d)$ \\
\hline $\mathrm{Pu}$ & $\begin{array}{l}\text { DI Water } \mathrm{pH} \\
8.3\end{array}$ & 219-S-2 & Batch & $1.80 \mathrm{E}+03$ & & 1 day & 7.70E-07 & 20 & $(\mathrm{c}, \mathrm{d})$ \\
\hline $\mathrm{Pu}$ & $\begin{array}{l}\text { DI Water pH } \\
9.4\end{array}$ & 219-S-2 & Batch & $1.80 \mathrm{E}+02$ & & 1 day & 7.70E-07 & 20 & $(\mathrm{c}, \mathrm{d})$ \\
\hline $\mathrm{Pu}$ & $\begin{array}{l}\text { DI Water } \mathrm{pH} \\
11.1\end{array}$ & 219-S-2 & Batch & $1.62 \mathrm{E}+02$ & & 1 day & $7.70 \mathrm{E}-07$ & 20 & $(\mathrm{c}, \mathrm{d})$ \\
\hline $\mathrm{Pu}$ & $\begin{array}{l}\text { DI Water } \mathrm{pH} \\
12.0 \\
\end{array}$ & 219-S-2 & Batch & $8.50 \mathrm{E}+01$ & & 1 day & 7.70E-07 & 20 & $(c, d)$ \\
\hline $\mathrm{Pu}$ & $\begin{array}{l}\text { DI Water } \mathrm{pH} \\
12.9\end{array}$ & 219-S-2 & Batch & $2.00 \mathrm{E}+03$ & & 1 day & 7.70E-07 & 20 & $(\mathrm{c}, \mathrm{d})$ \\
\hline $\mathrm{Pu}$ & $\begin{array}{l}\text { DI Water } \mathrm{pH} \\
14.2\end{array}$ & 219-S-2 & Batch & $2.00 \mathrm{E}+03$ & & 1 day & 7.70E-07 & 20 & $(\mathrm{c}, \mathrm{d})$ \\
\hline \multicolumn{10}{|c|}{$\begin{array}{l}\text { (a) Delegard and Barney } 1983 . \\
\text { (b) Hajek and Knoll } 1966 . \\
\text { (c) Rhodes } 1952 . \\
\text { (d) Rhodes } 1957 . \\
\text { *Data is best estimate. }\end{array}$} \\
\hline
\end{tabular}


Table 9. Contaminant Distribution Coefficient Data for Strontium

\begin{tabular}{|c|c|c|c|c|c|c|c|c|c|}
\hline Species & $\begin{array}{c}\text { Aqueous } \\
\text { Phase }\end{array}$ & Solid Phase & Method & $\begin{array}{c}\mathrm{Kd} \\
(\mathrm{mL} / \mathrm{g})\end{array}$ & Std. Dev. & $\begin{array}{l}\text { Equil. } \\
\text { Time }\end{array}$ & $\begin{array}{c}\text { In. Soln. } \\
\text { Conc. }\end{array}$ & $\begin{array}{l}\text { Solution/ } \\
\text { Solid } \\
(\mathrm{mL} / \mathrm{g})\end{array}$ & Reference \\
\hline $\operatorname{Sr}(\mathrm{II})$ & Hanford GW & CGS-1 & Batch & 17.9 & 1.3 & 4 days & Trace & 30 & (a) \\
\hline $\mathrm{Sr}(\mathrm{II})$ & Hanford GW & CGS-1 & Batch & 18.8 & 2.9 & 14 days & Trace & 30 & (a) \\
\hline $\mathrm{Sr}(\mathrm{II})$ & Hanford GW & CGS-1 & Batch & 20.7 & 3.2 & 35 days & Trace & 30 & (a) \\
\hline $\mathrm{Sr}(\mathrm{II})$ & $\begin{array}{l}\text { Acid Proc } \\
\text { Waste }\end{array}$ & CGS-1 & Batch & 7.4 & 1.2 & 4 days & Trace & 30 & (a) \\
\hline $\mathrm{Sr}(\mathrm{II})$ & $\begin{array}{l}\text { Acid Proc } \\
\text { Waste }\end{array}$ & CGS-1 & Batch & 11.7 & 0.8 & 14 days & Trace & 30 & (a) \\
\hline $\operatorname{Sr}(\mathrm{II})$ & $\begin{array}{l}\text { Acid Proc } \\
\text { Waste }\end{array}$ & CGS-1 & Batch & 13 & 0.7 & 35 days & Trace & 30 & (a) \\
\hline $\mathrm{Sr}(\mathrm{II})$ & Hanford GW & TBS-1 & Batch & 35.4 & 14.7 & 4 days & Trace & 30 & (a) \\
\hline $\operatorname{Sr}(\mathrm{II})$ & Hanford GW & TBS-1 & Batch & 18.2 & 2.1 & 14 days & Trace & 30 & (a) \\
\hline $\mathrm{Sr}(\mathrm{II})$ & Hanford GW & TBS-1 & Batch & 16.1 & 6.8 & 35 days & Trace & 30 & (a) \\
\hline $\mathrm{Sr}(\mathrm{II})$ & $\begin{array}{l}\text { Acid Proc } \\
\text { Waste }\end{array}$ & TBS-1 & Batch & 8.1 & 6 & 4 days & Trace & 30 & (a) \\
\hline $\operatorname{Sr}(\mathrm{II})$ & $\begin{array}{l}\text { Acid Proc } \\
\text { Waste }\end{array}$ & TBS-1 & Batch & 9.2 & 2.2 & 14 days & Trace & 30 & (a) \\
\hline $\mathrm{Sr}(\mathrm{II})$ & $\begin{array}{l}\text { Acid Proc } \\
\text { Waste }\end{array}$ & TBS-1 & Batch & 7.8 & 1 & 35 days & Trace & 30 & (a) \\
\hline $\mathrm{Sr}(\mathrm{II})$ & Hanford GW & Trench- 8 & Batch & 23.4 & 4.1 & 5 days & Trace & 30 & (a) \\
\hline $\mathrm{Sr}(\mathrm{II})$ & Hanford GW & Trench-8 & Batch & 18.1 & 4 & 44 days & Trace & 30 & (a) \\
\hline $\mathrm{Sr}(\mathrm{II})$ & Solution 1 & Soil A & Batch & 133 & 13 & 8.5 days & Trace & 30 & (b) \\
\hline $\mathrm{Sr}(\mathrm{II})$ & Solution 2 & Soil A & Batch & 238 & 21 & 8.5 days & Trace & 30 & (b) \\
\hline $\mathrm{Sr}(\mathrm{II})$ & Solution 3 & Soil A & Batch & 40 & 3 & 8.5 days & Trace & 30 & (b) \\
\hline $\operatorname{Sr}(\mathrm{II})$ & Solution 4 & Soil A & Batch & 86 & 8 & 8.5 days & Trace & 30 & (b) \\
\hline $\operatorname{Sr}(\mathrm{II})$ & Solution 5 & Soil A & Batch & 222 & 28 & 8.5 days & Trace & 30 & (b) \\
\hline $\mathrm{Sr}(\mathrm{II})$ & Solution 6 & Soil A & Batch & 17 & 1 & 8.5 days & Trace & 30 & (b) \\
\hline $\operatorname{Sr}(\mathrm{II})$ & Solution 1 & Soil A & Batch & 112 & 1 & 21.5 days & \begin{tabular}{|l|} 
Trace \\
\end{tabular} & 30 & (b) \\
\hline $\mathrm{Sr}(\mathrm{II})$ & Solution 2 & Soil A & Batch & 181 & 12 & 21.5 days & Trace & 30 & (b) \\
\hline $\operatorname{Sr}(\mathrm{II})$ & Solution 3 & Soil A & Batch & 38 & 8 & 21.5 days & Trace & 30 & (b) \\
\hline $\mathrm{Sr}(\mathrm{II})$ & Solution 4 & Soil A & Batch & 74 & 3 & 21.5 days & Trace & 30 & (b) \\
\hline $\operatorname{Sr}(\mathrm{II})$ & Solution 5 & Soil A & Batch & 207 & 18 & 21.5 days & Trace & 30 & (b) \\
\hline $\operatorname{Sr}(\mathrm{II})$ & Solution 6 & Soil A & Batch & 21 & 3 & 21.5 days & Trace & 30 & (b) \\
\hline
\end{tabular}




\begin{tabular}{|c|c|c|c|c|c|c|c|c|c|}
\hline Species & $\begin{array}{l}\text { Aqueous } \\
\text { Phase }\end{array}$ & Solid Phase & Method & $\begin{array}{c}\mathrm{Kd} \\
(\mathrm{mL} / \mathrm{g})\end{array}$ & Std. Dev. & $\begin{array}{c}\text { Equil. } \\
\text { Time }\end{array}$ & $\begin{array}{c}\text { In. Soln. } \\
\text { Conc. }\end{array}$ & $\begin{array}{l}\text { Solution/ } \\
\text { Solid } \\
(\mathrm{mL} / \mathrm{g})\end{array}$ & Reference \\
\hline $\operatorname{Sr}(\mathrm{II})$ & Solution 1 & Soil B & Batch & 129 & 15 & 8.5 days & Trace & 30 & (b) \\
\hline $\operatorname{Sr}(\mathrm{II})$ & Solution 2 & Soil B & Batch & 170 & 27 & 8.5 days & Trace & 30 & (b) \\
\hline $\operatorname{Sr}(\mathrm{II})$ & Solution 3 & Soil B & Batch & 31 & 3 & 8.5 days & Trace & 30 & (b) \\
\hline $\operatorname{Sr}(\mathrm{II})$ & Solution 4 & Soil B & Batch & 47 & 4 & 8.5 days & Trace & 30 & (b) \\
\hline $\mathrm{Sr}(\mathrm{II})$ & Solution 5 & Soil B & Batch & 36 & 2 & 8.5 days & Trace & 30 & (b) \\
\hline $\mathrm{Sr}(\mathrm{II})$ & Solution 6 & Soil B & Batch & 9 & 3 & 8.5 days & Trace & 30 & (b) \\
\hline $\operatorname{Sr}(\mathrm{II})$ & Solution 1 & Soil B & Batch & 114 & 9 & 21.5 days & Trace & 30 & (b) \\
\hline $\operatorname{Sr}(\mathrm{II})$ & Solution 2 & Soil B & Batch & 132 & 11 & 21.5 days & Trace & 30 & (b) \\
\hline $\operatorname{Sr}(\mathrm{II})$ & Solution 3 & Soil B & Batch & 38 & 1 & 21.5 days & Trace & 30 & (b) \\
\hline $\mathrm{Sr}(\mathrm{II})$ & Solution 4 & Soil B & Batch & 39 & 2 & 21.5 days & Trace & 30 & (b) \\
\hline $\operatorname{Sr}(\mathrm{II})$ & Solution 5 & Soil B & Batch & 37 & 1 & 21.5 days & Trace & 30 & (b) \\
\hline $\operatorname{Sr}(\mathrm{II})$ & Solution 6 & Soil B & Batch & 12 & 2 & 21.5 days & Trace & 30 & (b) \\
\hline $\mathrm{Sr}(\mathrm{II})$ & Solution L & Soil B & Batch & 6 & 1 & 7 days & Trace & 30 & (b) \\
\hline $\mathrm{Sr}(\mathrm{II})$ & Solution H & Soil B & Batch & 1 & 0.2 & 7 days & Trace & 30 & (b) \\
\hline $\operatorname{Sr}(\mathrm{II})$ & Solution H & Soil B & Column & 0.8 & & 4 days & Trace & 30 & (b) \\
\hline $\mathrm{Sr}(\mathrm{II})$ & Hanford GW2 & B850007A & Batch & 14.09 & 0.83 & 14 days & Trace & 30 & (c) \\
\hline $\operatorname{Sr}(\mathrm{II})$ & Hanford GW2 & B850010A & Batch & 14.25 & 0.49 & 14 days & Trace & 30 & (c) \\
\hline $\operatorname{Sr}(\mathrm{II})$ & Hanford GW2 & B850012A & Batch & 13.81 & 0.78 & 14 days & Trace & 30 & (c) \\
\hline $\mathrm{Sr}(\mathrm{II})$ & Hanford GW2 & B850014A & Batch & 13.94 & 0.5 & 14 days & Trace & 30 & (c) \\
\hline $\operatorname{Sr}(\mathrm{II})$ & Hanford GW2 & B850015A & Batch & 13.64 & 0.85 & 14 days & Trace & 30 & (c) \\
\hline $\mathrm{Sr}(\mathrm{II})$ & Hanford GW2 & B850016A & Batch & 12.81 & 0.33 & 14 days & Trace & 30 & (c) \\
\hline $\operatorname{Sr}(\mathrm{II})$ & Hanford GW2 & B850017A & Batch & 15.46 & 0.33 & 14 days & Trace & 30 & (c) \\
\hline $\operatorname{Sr}(\mathrm{II})$ & Hanford GW2 & B850019A & Batch & 14.25 & 0.45 & 14 days & Trace & 30 & (c) \\
\hline $\mathrm{Sr}(\mathrm{II})$ & Hanford GW2 & B850020A & Batch & 15.32 & 0.37 & 14 days & Trace & 30 & (c) \\
\hline $\mathrm{Sr}(\mathrm{II})$ & Hanford GW2 & B850021A & Batch & 14.91 & 0.63 & 14 days & Trace & 30 & (c) \\
\hline $\mathrm{Sr}(\mathrm{II})$ & Hanford GW2 & B850022A & Batch & 12.18 & 0.29 & 14 days & Trace & 30 & (c) \\
\hline $\operatorname{Sr}(\mathrm{II})$ & Hanford GW2 & B850023A & Batch & 13.04 & 0.95 & 14 days & Trace & 30 & (c) \\
\hline $\mathrm{Sr}(\mathrm{II})$ & Hanford GW2 & B850024A & Batch & 16.92 & 0.92 & 14 days & Trace & 30 & (c) \\
\hline $\operatorname{Sr}(\mathrm{II})$ & Hanford GW2 & B850025A & Batch & 12.35 & 0.85 & 14 days & Trace & 30 & (c) \\
\hline $\mathrm{Sr}(\mathrm{II})$ & Hanford GW2 & B850027A & Batch & 16.17 & 1.42 & 14 days & Trace & 30 & (c) \\
\hline $\mathrm{Sr}(\mathrm{II})$ & Hanford GW2 & B850029A & Batch & 14.22 & 0.34 & 14 days & Trace & 30 & (c) \\
\hline $\operatorname{Sr}(\mathrm{II})$ & Hanford GW2 & B850031A & Batch & 16.9 & 0.11 & 14 days & Trace & 30 & (c) \\
\hline $\mathrm{Sr}(\mathrm{II})$ & Hanford GW2 & B850032A & Batch & 16.89 & 1.72 & 14 days & Trace & 30 & (c) \\
\hline
\end{tabular}




\begin{tabular}{|c|c|c|c|c|c|c|c|c|c|}
\hline Species & $\begin{array}{c}\text { Aqueous } \\
\text { Phase }\end{array}$ & Solid Phase & Method & $\begin{array}{c}\mathrm{Kd} \\
(\mathrm{mL} / \mathrm{g})\end{array}$ & Std. Dev. & $\begin{array}{c}\text { Equil. } \\
\text { Time }\end{array}$ & $\begin{array}{c}\text { In. Soln. } \\
\text { Conc. }\end{array}$ & $\begin{array}{l}\text { Solution/ } \\
\text { Solid } \\
(\mathrm{mL} / \mathrm{g})\end{array}$ & Reference \\
\hline $\operatorname{Sr}(\mathrm{II})$ & Hanford GW2 & B850034A & Batch & 16.92 & 1.53 & 14 days & Trace & 30 & (c) \\
\hline $\operatorname{Sr}(\mathrm{II})$ & Hanford GW2 & B850035A & Batch & 16.79 & 2.68 & 14 days & Trace & 30 & (c) \\
\hline $\mathrm{Sr}(\mathrm{II})$ & $0.038 \mathrm{M} \mathrm{CaCl}_{2}$ & Trench-8 & \begin{tabular}{|l|} 
Col \\
$100 \%$ Sat
\end{tabular} & 1.8 & & & Trace & & (d) \\
\hline $\operatorname{Sr}(\mathrm{II})$ & $0.038 \mathrm{M} \mathrm{CaCl}_{2}$ & Trench-8 & \begin{tabular}{|l|} 
Col \\
$100 \%$ Sat
\end{tabular} & 1.4 & & & Trace & & (d) \\
\hline $\operatorname{Sr}(\mathrm{II})$ & $0.038 \mathrm{M} \mathrm{CaCl}_{2}$ & Trench-8 & $\begin{array}{l}\text { Col } \\
72 \% \text { Sat }\end{array}$ & 2 & & & Trace & & (d) \\
\hline $\operatorname{Sr}(\mathrm{II})$ & $0.038 \mathrm{M} \mathrm{CaCl}_{2}$ & Trench-8 & $\begin{array}{l}\text { Col } \\
47 \% \text { Sat } \\
\end{array}$ & 1 & & & Trace & & (d) \\
\hline $\operatorname{Sr}(\mathrm{II})$ & $0.038 \mathrm{M} \mathrm{CaCl}_{2}$ & Trench-8 & $\begin{array}{l}\text { Col } \\
26 \% \text { Sat } \\
\text { UFA }\end{array}$ & 0.7 & & & Trace & & (d) \\
\hline $\operatorname{Sr}(\mathrm{II})$ & $0.038 \mathrm{M} \mathrm{CaCl}_{2}$ & Trench-8 & \begin{tabular}{|l} 
Col \\
$26 \%$ Sat \\
UFA
\end{tabular} & 0.8 & & & Trace & & (d) \\
\hline $\mathrm{Sr}(\mathrm{II})$ & Soln 1 & Sed L & Batch & -0.89 & & 7 days & Trace & 5 & (e) \\
\hline $\operatorname{Sr}(\mathrm{II})$ & Soln 2 & Sed L & Batch & -0.15 & & 7 days & Trace & 5 & (e) \\
\hline $\operatorname{Sr}(\mathrm{II})$ & Soln 3 & Sed L & Batch & 0.46 & & 7 days & Trace & 5 & (e) \\
\hline $\mathrm{Sr}(\mathrm{II})$ & Soln 4 & Sed L & Batch & 0.59 & & 7 days & Trace & 5 & (e) \\
\hline $\mathrm{Sr}(\mathrm{II})$ & Soln 5 & Sed L & Batch & 51.4 & & 7 days & Trace & 5 & (e) \\
\hline $\mathrm{Sr}(\mathrm{II})$ & Soln 6 & Sed L & Batch & 21.1 & & 7 days & Trace & 5 & (e) \\
\hline $\operatorname{Sr}(\mathrm{II})$ & Soln 7 & Sed L & Batch & 19.7 & & 7 days & Trace & 5 & (e) \\
\hline $\operatorname{Sr}(\mathrm{II})$ & Soln 8 & Sed L & Batch & 0.51 & & 7 days & Trace & 5 & (e) \\
\hline $\mathrm{Sr}(\mathrm{II})$ & Soln 9 & Sed L & Batch & 0.23 & & 7 days & Trace & 5 & (e) \\
\hline $\mathrm{Sr}(\mathrm{II})$ & Soln 10 & Sed L & Batch & 0.67 & & 7 days & Trace & 5 & (e) \\
\hline $\operatorname{Sr}(\mathrm{II})$ & Soln 11 & Sed L & Batch & -0.08 & & 7 days & Trace & 5 & (e) \\
\hline $\mathrm{Sr}(\mathrm{II})$ & Soln 12 & Sed L & Batch & 0.27 & & 7 days & Trace & 5 & (e) \\
\hline $\mathrm{Sr}(\mathrm{II})$ & Soln 13 & Sed L & Batch & 0.6 & & 7 days & Trace & 5 & (e) \\
\hline $\mathrm{Sr}(\mathrm{II})$ & Soln 14 & Sed L & Batch & 493 & & 7 days & Trace & 5 & (e) \\
\hline $\mathrm{Sr}(\mathrm{II})$ & Soln 15 & Sed L & Batch & 0.29 & & 7 days & Trace & 5 & (e) \\
\hline $\mathrm{Sr}(\mathrm{II})$ & Soln 16 & Sed L & Batch & 0.13 & & 7 days & Trace & 5 & (e) \\
\hline $\operatorname{Sr}(\mathrm{II})$ & Soln 17 & Sed L & Batch & 0.06 & & 7 days & Trace & 5 & (e) \\
\hline $\mathrm{Sr}(\mathrm{II})$ & Soln 18 & Sed L & Batch & 0.56 & & 7 days & Trace & 5 & (e) \\
\hline $\mathrm{Sr}(\mathrm{II})$ & Soln 19 & Sed L & Batch & 0.63 & & 7 days & Trace & 5 & (e) \\
\hline $\mathrm{Sr}(\mathrm{II})$ & Soln 20 & Sed L & Batch & 49 & & 7 days & Trace & 5 & (e) \\
\hline $\mathrm{Sr}(\mathrm{II})$ & Soln 1 & Sed P & Batch & -0.43 & & 7 days & Trace & 5 & (e) \\
\hline $\mathrm{Sr}(\mathrm{II})$ & Soln 2 & Sed P & Batch & -0.22 & & 7 days & Trace & 5 & (e) \\
\hline
\end{tabular}




\begin{tabular}{|c|c|c|c|c|c|c|c|c|c|}
\hline Species & $\begin{array}{c}\text { Aqueous } \\
\text { Phase }\end{array}$ & Solid Phase & Method & $\begin{array}{c}\mathrm{Kd} \\
(\mathrm{mL} / \mathrm{g})\end{array}$ & Std. Dev. & $\begin{array}{c}\text { Equil. } \\
\text { Time }\end{array}$ & $\begin{array}{l}\text { In. Soln. } \\
\text { Conc. }\end{array}$ & $\begin{array}{c}\text { Solution/ } \\
\text { Solid } \\
(\mathrm{mL} / \mathrm{g}) \\
\end{array}$ & Reference \\
\hline $\operatorname{Sr}(\mathrm{II})$ & Soln 3 & Sed P & Batch & 0.92 & & 7 days & Trace & 5 & (e) \\
\hline $\operatorname{Sr}(\mathrm{II})$ & Soln 4 & Sed P & Batch & 0.6 & & 7 days & Trace & 5 & (e) \\
\hline $\mathrm{Sr}(\mathrm{II})$ & Soln 5 & Sed P & Batch & 45.2 & & 7 days & Trace & 5 & (e) \\
\hline $\operatorname{Sr}(\mathrm{II})$ & Soln 6 & Sed P & Batch & 28.7 & & 7 days & Trace & 5 & (e) \\
\hline $\mathrm{Sr}(\mathrm{II})$ & Soln 7 & Sed P & Batch & 16.8 & & 7 days & Trace & 5 & (e) \\
\hline $\operatorname{Sr}(\mathrm{II})$ & Soln 8 & Sed P & Batch & 0.79 & & 7 days & Trace & 5 & (e) \\
\hline $\mathrm{Sr}(\mathrm{II})$ & Soln 9 & Sed P & Batch & 0.34 & & 7 days & Trace & 5 & (e) \\
\hline $\mathrm{Sr}(\mathrm{II})$ & Soln 10 & Sed P & Batch & 0.82 & & 7 days & Trace & 5 & (e) \\
\hline $\operatorname{Sr}(\mathrm{II})$ & Soln 11 & Sed P & Batch & 0.21 & & 7 days & Trace & 5 & (e) \\
\hline $\mathrm{Sr}(\mathrm{II})$ & Soln 12 & Sed P & Batch & 0.24 & & 7 days & Trace & 5 & (e) \\
\hline $\mathrm{Sr}(\mathrm{II})$ & Soln 13 & Sed P & Batch & 0.5 & & 7 days & Trace & 5 & (e) \\
\hline $\mathrm{Sr}(\mathrm{II})$ & Soln 14 & Sed P & Batch & 487 & & 7 days & Trace & 5 & (e) \\
\hline $\mathrm{Sr}(\mathrm{II})$ & Soln 15 & Sed P & Batch & 0.12 & & 7 days & Trace & 5 & (e) \\
\hline $\mathrm{Sr}(\mathrm{II})$ & Soln 16 & Sed P & Batch & 0.06 & & 7 days & Trace & 5 & (e) \\
\hline $\mathrm{Sr}(\mathrm{II})$ & Soln 17 & Sed P & Batch & -0.32 & & 7 days & Trace & 5 & (e) \\
\hline $\operatorname{Sr}(\mathrm{II})$ & Soln 18 & Sed P & Batch & 0.33 & & 7 days & Trace & 5 & (e) \\
\hline $\mathrm{Sr}(\mathrm{II})$ & Soln 19 & Sed P & Batch & 0.25 & & 7 days & Trace & 5 & (e) \\
\hline $\mathrm{Sr}(\mathrm{II})$ & Soln 20 & Sed P & Batch & 50.5 & & 7 days & Trace & 5 & (e) \\
\hline $\operatorname{Sr}(\mathrm{II})$ & Soln 1 & Sed S & Batch & 0.5 & & 7 days & Trace & 5 & (e) \\
\hline $\mathrm{Sr}(\mathrm{II})$ & Soln 2 & Sed S & Batch & 0.14 & & 7 days & Trace & 5 & (e) \\
\hline $\mathrm{Sr}(\mathrm{II})$ & Soln 3 & Sed S & Batch & 2.29 & & 7 days & Trace & 5 & (e) \\
\hline $\mathrm{Sr}(\mathrm{II})$ & Soln 4 & Sed S & Batch & 1.48 & & 7 days & Trace & 5 & (e) \\
\hline $\mathrm{Sr}(\mathrm{II})$ & Soln 5 & Sed S & Batch & 313 & & 7 days & Trace & 5 & (e) \\
\hline $\mathrm{Sr}(\mathrm{II})$ & Soln 6 & Sed S & Batch & 71 & & 7 days & Trace & 5 & (e) \\
\hline $\operatorname{Sr}(\mathrm{II})$ & Soln 7 & Sed S & Batch & 60.6 & & 7 days & Trace & 5 & (e) \\
\hline $\mathrm{Sr}(\mathrm{II})$ & Soln 8 & Sed S & Batch & 1.47 & & 7 days & Trace & 5 & (e) \\
\hline $\mathrm{Sr}(\mathrm{II})$ & Soln 9 & Sed S & Batch & 0.27 & & 7 days & Trace & 5 & (e) \\
\hline $\mathrm{Sr}(\mathrm{II})$ & Soln 10 & Sed S & Batch & 1.61 & & 7 days & Trace & 5 & (e) \\
\hline $\operatorname{Sr}(\mathrm{II})$ & Soln 11 & Sed S & Batch & 0.87 & & 7 days & Trace & 5 & (e) \\
\hline $\mathrm{Sr}(\mathrm{II})$ & Soln 12 & Sed S & Batch & 0.77 & & 7 days & Trace & 5 & (e) \\
\hline $\mathrm{Sr}(\mathrm{II})$ & Soln 13 & Sed S & Batch & 0.95 & & 7 days & Trace & 5 & (e) \\
\hline $\mathrm{Sr}(\mathrm{II})$ & Soln 14 & Sed S & Batch & 402 & & 7 days & Trace & 5 & (e) \\
\hline $\mathrm{Sr}(\mathrm{II})$ & Soln 15 & Sed S & Batch & 1.24 & & 7 days & Trace & 5 & (e) \\
\hline $\mathrm{Sr}(\mathrm{II})$ & Soln 16 & Sed S & Batch & 0.33 & & 7 days & Trace & 5 & (e) \\
\hline $\operatorname{Sr}(\mathrm{II})$ & Soln 17 & Sed S & Batch & 0.06 & & 7 days & Trace & 5 & (e) \\
\hline $\mathrm{Sr}(\mathrm{II})$ & Soln 18 & Sed S & Batch & 0.26 & & 7 days & Trace & 5 & (e) \\
\hline
\end{tabular}




\begin{tabular}{|c|c|c|c|c|c|c|c|c|c|}
\hline Species & $\begin{array}{c}\text { Aqueous } \\
\text { Phase }\end{array}$ & Solid Phase & Method & $\begin{array}{c}\mathrm{Kd} \\
(\mathrm{mL} / \mathrm{g})\end{array}$ & Std. Dev. & $\begin{array}{c}\text { Equil. } \\
\text { Time }\end{array}$ & $\begin{array}{c}\text { In. Soln. } \\
\text { Conc. }\end{array}$ & $\begin{array}{l}\text { Solution/ } \\
\text { Solid } \\
(\mathrm{mL} / \mathrm{g})\end{array}$ & Reference \\
\hline $\mathrm{Sr}(\mathrm{II})$ & Soln 19 & Sed S & Batch & 1.05 & & 7 days & Trace & 5 & (e) \\
\hline $\mathrm{Sr}(\mathrm{II})$ & Soln 20 & Sed S & Batch & 128 & & 7 days & Trace & 5 & (e) \\
\hline $\operatorname{Sr}(\mathrm{II})$ & $\begin{array}{l}\text { REDOX } \\
\text { Liquor }\end{array}$ & MSG-1 & Batch & 4 & & 4 days & Trace & 10 & (f) \\
\hline $\mathrm{Sr}(\mathrm{II})$ & $\begin{array}{l}\text { REDOX } \\
\text { Liquor }\end{array}$ & MSG-1 & Batch & 28 & & 4 days & Trace & 10 & (f) \\
\hline $\operatorname{Sr}(\mathrm{II})$ & $\begin{array}{l}\text { REDOX } \\
\text { Liquor }\end{array}$ & MSG-1 & Batch & 0.2 & 0.2 & 11 days & Trace & 10 & (f) \\
\hline $\operatorname{Sr}(\mathrm{II})$ & Groundwater & \begin{tabular}{|l} 
Burbank \\
loamy fine \\
sand
\end{tabular} & Batch & 48 & & $16 \mathrm{hrs}$ & Trace & 100 & (g) \\
\hline $\operatorname{Sr}(\mathrm{II})$ & Groundwater & \begin{tabular}{|l|} 
Burbank \\
loamy fine \\
sand
\end{tabular} & Batch & 52 & & $16 \mathrm{hrs}$ & Trace & 50 & (g) \\
\hline $\operatorname{Sr}(\mathrm{II})$ & Groundwater & \begin{tabular}{|l|} 
Burbank \\
loamy fine \\
sand
\end{tabular} & Batch & 51 & & $16 \mathrm{hrs}$ & Trace & 10 & (g) \\
\hline $\operatorname{Sr}(\mathrm{II})$ & Groundwater & \begin{tabular}{|l|} 
Burbank \\
loamy fine \\
sand \\
\end{tabular} & Batch & 43 & & $16 \mathrm{hrs}$ & Trace & 5 & (g) \\
\hline $\operatorname{Sr}(\mathrm{II})$ & $3 \mathrm{M} \mathrm{NaNO}_{3}$ & \begin{tabular}{|l} 
Burbank \\
loamy fine \\
sand
\end{tabular} & Batch & 2.1 & & $16 \mathrm{hrs}$ & Trace & 100 & (g) \\
\hline $\operatorname{Sr}(\mathrm{II})$ & $3 \mathrm{M} \mathrm{NaNO}_{3}$ & \begin{tabular}{|l} 
Burbank \\
loamy fine \\
sand
\end{tabular} & Batch & 0.78 & & $16 \mathrm{hrs}$ & Trace & 50 & (g) \\
\hline $\mathrm{Sr}(\mathrm{II})$ & $3 \mathrm{M} \mathrm{NaNO}_{3}$ & \begin{tabular}{|l} 
Burbank \\
loamy fine \\
sand
\end{tabular} & Batch & 0.5 & & $16 \mathrm{hrs}$ & Trace & 10 & (g) \\
\hline $\operatorname{Sr}(\mathrm{II})$ & $3 \mathrm{M} \mathrm{NaNO}_{3}$ & \begin{tabular}{|l} 
Burbank \\
loamy fine \\
sand
\end{tabular} & Batch & 0.38 & & $16 \mathrm{hrs}$ & Trace & 5 & (g) \\
\hline $\operatorname{Sr}(\mathrm{II})$ & $0.5 \mathrm{M} \mathrm{NaCl}$ & \begin{tabular}{|l} 
Burbank \\
loamy fine \\
sand
\end{tabular} & Batch & 7.3 & & $16 \mathrm{hrs}$ & Trace & 100 & (g) \\
\hline $\mathrm{Sr}(\mathrm{II})$ & $0.5 \mathrm{M} \mathrm{NaCl}$ & $\begin{array}{l}\text { Burbank } \\
\text { loamy fine } \\
\text { sand }\end{array}$ & Batch & 3.98 & & $16 \mathrm{hrs}$ & Trace & 50 & (g) \\
\hline $\mathrm{Sr}(\mathrm{II})$ & $0.5 \mathrm{M} \mathrm{NaCl}$ & $\begin{array}{l}\text { Burbank } \\
\text { loamy fine } \\
\text { sand }\end{array}$ & Batch & 1.98 & & $16 \mathrm{hrs}$ & Trace & 10 & (g) \\
\hline
\end{tabular}




\begin{tabular}{|c|c|c|c|c|c|c|c|c|c|}
\hline Species & $\begin{array}{c}\text { Aqueous } \\
\text { Phase }\end{array}$ & Solid Phase & Method & $\begin{array}{c}\mathrm{Kd} \\
(\mathrm{mL} / \mathrm{g})\end{array}$ & Std. Dev. & $\begin{array}{c}\text { Equil. } \\
\text { Time }\end{array}$ & $\begin{array}{c}\text { In. Soln. } \\
\text { Conc. }\end{array}$ & $\begin{array}{l}\text { Solution/ } \\
\text { Solid } \\
(\mathrm{mL} / \mathrm{g})\end{array}$ & Reference \\
\hline $\mathrm{Sr}(\mathrm{II})$ & $0.5 \mathrm{M} \mathrm{NaCl}$ & $\begin{array}{l}\text { Burbank } \\
\text { loamy fine } \\
\text { sand }\end{array}$ & Batch & 1.49 & & $16 \mathrm{hrs}$ & Trace & 5 & (g) \\
\hline $\operatorname{Sr}(\mathrm{II})$ & $3 \mathrm{M} \mathrm{NaOAc}$ & \begin{tabular}{|l} 
Burbank \\
loamy fine \\
sand
\end{tabular} & Batch & 2.23 & & $16 \mathrm{hrs}$ & Trace & 100 & (g) \\
\hline $\operatorname{Sr}(\mathrm{II})$ & $3 \mathrm{M} \mathrm{NaOAc}$ & $\begin{array}{l}\text { Burbank } \\
\text { loamy fine } \\
\text { sand }\end{array}$ & Batch & 1.69 & & $16 \mathrm{hrs}$ & Trace & 50 & (g) \\
\hline $\operatorname{Sr}(\mathrm{II})$ & $3 \mathrm{M} \mathrm{NaOAc}$ & \begin{tabular}{|l|} 
Burbank \\
loamy fine \\
sand
\end{tabular} & Batch & 1.02 & & $16 \mathrm{hrs}$ & Trace & 10 & (g) \\
\hline $\operatorname{Sr}(\mathrm{II})$ & $3 \mathrm{M} \mathrm{NaOAc}$ & \begin{tabular}{|l|} 
Burbank \\
loamy fine \\
sand
\end{tabular} & Batch & 0.53 & & $16 \mathrm{hrs}$ & Trace & 5 & (g) \\
\hline $\mathrm{Sr}(\mathrm{II})$ & $0.2 \mathrm{M} \mathrm{NaCl}$ & Ritzville A12 & Batch & 21 & & Unknown & & & (h) \\
\hline $\mathrm{Sr}(\mathrm{II})$ & $0.2 \mathrm{M} \mathrm{NaCl}$ & Ritzville B1 & Batch & 27 & & Unknown & & & (h) \\
\hline $\operatorname{Sr}(\mathrm{II})$ & $0.2 \mathrm{M} \mathrm{NaCl}$ & Ritzville C & Batch & 27 & & Unknown & & & (h) \\
\hline $\mathrm{Sr}(\mathrm{II})$ & $0.2 \mathrm{M} \mathrm{NaCl}$ & Ritzville Cca & Batch & 24 & & Unknown & & & (h) \\
\hline $\mathrm{Sr}(\mathrm{II})$ & $0.2 \mathrm{M} \mathrm{NaCl}$ & Burbank A12 & Batch & 12 & & Unknown & & & (h) \\
\hline $\mathrm{Sr}(\mathrm{II})$ & $0.2 \mathrm{M} \mathrm{NaCl}$ & Burbank AC & Batch & 15 & & Unknown & & & (h) \\
\hline $\mathrm{Sr}(\mathrm{II})$ & $0.2 \mathrm{M} \mathrm{NaCl}$ & Burbank AC2 & Batch & 21 & & Unknown & & & (h) \\
\hline $\mathrm{Sr}(\mathrm{II})$ & $0.2 \mathrm{M} \mathrm{NaCl}$ & Burbank IC & Batch & 17 & & Unknown & & & (h) \\
\hline $\mathrm{Sr}(\mathrm{II})$ & $0.2 \mathrm{M} \mathrm{NaCl}$ & Ephrata A12 & Batch & 12 & & Unknown & & & (h) \\
\hline $\operatorname{Sr}(\mathrm{II})$ & $0.2 \mathrm{M} \mathrm{NaCl}$ & Ephrata B1 & Batch & 12 & & Unknown & & & (h) \\
\hline $\mathrm{Sr}(\mathrm{II})$ & $0.2 \mathrm{M} \mathrm{NaCl}$ & Ephrata IB2 & Batch & 17 & & Unknown & & & (h) \\
\hline $\mathrm{Sr}(\mathrm{II})$ & $0.2 \mathrm{M} \mathrm{NaCl}$ & Ephrata IIC & Batch & 23 & & Unknown & & & (h) \\
\hline $\mathrm{Sr}(\mathrm{II})$ & T-106 Soln. 1 & Sed 1 & Batch & 3.91 & & 1-2 wks* & Trace* & $20 *$ & $(f, i)$ \\
\hline $\mathrm{Sr}(\mathrm{II})$ & T-106 Soln. 2 & Sed 1 & Batch & 1.74 & & 1-2 wks* & Trace* & $20 *$ & $(f, i)$ \\
\hline $\operatorname{Sr}(\mathrm{II})$ & T-106 Soln. 3 & Sed 1 & Batch & 0.023 & & 1-2 wks* & Trace* & $20 *$ & $(f, i)$ \\
\hline $\mathrm{Sr}(\mathrm{II})$ & T-106 Soln. 4 & Sed 1 & Batch & 0 & & 1-2 wks* & Trace* & $20^{*}$ & $(f, i)$ \\
\hline $\operatorname{Sr}(\mathrm{II})$ & T-106 Soln. 5 & Sed 1 & Batch & 18.4 & & $1-2$ wks* & Trace* & $20 *$ & $(f, i)$ \\
\hline $\operatorname{Sr}(\mathrm{II})$ & T-106 Soln. 1 & Sed 2 & Batch & 2.9 & & 1-2 wks* & Trace* & $20 *$ & $(\mathrm{f}, \mathrm{i})$ \\
\hline $\mathrm{Sr}(\mathrm{II})$ & T-106 Soln. 2 & Sed 2 & Batch & 1.17 & & 1-2 wks* & Trace* & $20 *$ & $(f, i)$ \\
\hline $\mathrm{Sr}(\mathrm{II})$ & T-106 Soln. 3 & Sed 2 & Batch & 0.047 & & 1-2 wks* & Trace* & $20 *$ & $(\mathrm{f}, \mathrm{i})$ \\
\hline
\end{tabular}




\begin{tabular}{|c|c|c|c|c|c|c|c|c|c|}
\hline Species & $\begin{array}{l}\text { Aqueous } \\
\text { Phase }\end{array}$ & Solid Phase & Method & $\begin{array}{c}\mathrm{Kd} \\
(\mathrm{mL} / \mathrm{g})\end{array}$ & Std. Dev. & $\begin{array}{c}\text { Equil. } \\
\text { Time }\end{array}$ & $\begin{array}{c}\text { In. Soln. } \\
\text { Conc. }\end{array}$ & $\begin{array}{l}\text { Solution/ } \\
\text { Solid } \\
(\mathrm{mL} / \mathrm{g})\end{array}$ & Reference \\
\hline $\mathrm{Sr}(\mathrm{II})$ & T-106 Soln. 4 & Sed 2 & Batch & 0 & & $1-2$ wks* & Trace* & $20^{*}$ & $(f, i)$ \\
\hline $\operatorname{Sr}(\mathrm{II})$ & T-106 Soln. 5 & Sed 2 & Batch & 8.4 & & 1-2 wks* & Trace* & $20 *$ & $(f, i)$ \\
\hline $\mathrm{Sr}(\mathrm{II})$ & T-106 Soln. 1 & Sed 3 & Batch & 2.93 & & 1-2 wks* & Trace* & $20 *$ & $(f, i)$ \\
\hline $\mathrm{Sr}(\mathrm{II})$ & T-106 Soln. 2 & Sed 3 & Batch & 1.22 & & 1-2 wks* & Trace* & $20 *$ & $(f, i)$ \\
\hline $\operatorname{Sr}(\mathrm{II})$ & T-106 Soln. 3 & Sed 3 & Batch & 0.072 & & $1-2$ wks* & Trace* & $20 *$ & $(f, i)$ \\
\hline $\mathrm{Sr}(\mathrm{II})$ & T-106 Soln. 4 & Sed 3 & Batch & 0 & & 1-2 wks* & Trace* & $20^{*}$ & $(f, i)$ \\
\hline $\operatorname{Sr}(\mathrm{II})$ & T-106 Soln. 5 & Sed 3 & Batch & 12.9 & & $1-2 \mathrm{wks}^{*}$ & Trace* & $20 *$ & $(f, i)$ \\
\hline $\mathrm{Sr}(\mathrm{II})$ & T-106 Soln. 1 & Sed 4 & Batch & 12.4 & & 1-2 wks* & Trace* & $20 *$ & $(f, i)$ \\
\hline $\mathrm{Sr}(\mathrm{II})$ & T-106 Soln. 2 & Sed 4 & Batch & 5.44 & & 1-2 wks* & Trace* & $20 *$ & $(f, i)$ \\
\hline $\mathrm{Sr}(\mathrm{II})$ & T-106 Soln. 3 & Sed 4 & Batch & 0.074 & & 1-2 wks* & Trace* & $20 *$ & $(\mathrm{f}, \mathrm{i})$ \\
\hline $\mathrm{Sr}(\mathrm{II})$ & T-106 Soln. 4 & Sed 4 & Batch & 0 & & $1-2$ wks$^{*}$ & Trace* & $20 *$ & $(\mathrm{f}, \mathrm{i})$ \\
\hline $\mathrm{Sr}(\mathrm{II})$ & T-106 Soln. 5 & Sed 4 & Batch & 174 & & $1-2$ wks* & Trace* & $20 *$ & $(f, i)$ \\
\hline $\mathrm{Sr}(\mathrm{II})$ & T-106 Soln. 1 & Sed 5 & Batch & 1.87 & & 1-2 wks* & Trace* & $20^{*}$ & $(f, i)$ \\
\hline $\mathrm{Sr}(\mathrm{II})$ & T-106 Soln. 2 & Sed 5 & Batch & 0.81 & & 1-2 wks* & Trace* & $20 *$ & $(f, i)$ \\
\hline $\operatorname{Sr}(\mathrm{II})$ & T-106 Soln. 3 & Sed 5 & Batch & 0.13 & & $1-2$ wks* & Trace* & $20 *$ & $(f, i)$ \\
\hline $\mathrm{Sr}(\mathrm{II})$ & T-106 Soln. 4 & Sed 5 & Batch & 0.17 & & $1-2 \mathrm{wks}^{*}$ & Trace* & $20 *$ & $(f, i)$ \\
\hline $\mathrm{Sr}(\mathrm{II})$ & T-106 Soln. 5 & Sed 5 & Batch & 12.7 & & 1-2 wks* & Trace* & $20 *$ & $(f, i)$ \\
\hline $\mathrm{Sr}(\mathrm{II})$ & T-106 Soln. 1 & Sed 6 & Batch & 2.5 & & 1-2 wks* & Trace* & $20 *$ & $(f, i)$ \\
\hline $\mathrm{Sr}(\mathrm{II})$ & T-106 Soln. 2 & Sed 6 & Batch & 0.95 & & 1-2 wks* & Trace* & $20 *$ & $(f, i)$ \\
\hline $\mathrm{Sr}(\mathrm{II})$ & T-106 Soln. 3 & Sed 6 & Batch & 0.1 & & 1-2 wks* & Trace* & $20 *$ & $(f, i)$ \\
\hline $\mathrm{Sr}(\mathrm{II})$ & T-106 Soln. 4 & Sed 6 & Batch & 0.14 & & 1-2 wks* & Trace* & $20 *$ & $(f, i)$ \\
\hline $\mathrm{Sr}(\mathrm{II})$ & T-106 Soln. 5 & Sed 6 & Batch & 39.2 & & 1-2 wks* & Trace* & $20 *$ & $(f, i)$ \\
\hline $\mathrm{Sr}(\mathrm{II})$ & T-106 Soln. 1 & Sed 7 & Batch & 1.86 & & 1-2 wks* & Trace* & $20 *$ & $(f, i)$ \\
\hline $\operatorname{Sr}(\mathrm{II})$ & T-106 Soln. 2 & Sed 7 & Batch & 0.87 & & $1-2 \mathrm{wks}^{*}$ & Trace* & $20 *$ & $(f, i)$ \\
\hline $\mathrm{Sr}(\mathrm{II})$ & T-106 Soln. 3 & Sed 7 & Batch & 0.08 & & $1-2$ wks* & Trace* & $20 *$ & $(f, i)$ \\
\hline $\mathrm{Sr}(\mathrm{II})$ & T-106 Soln. 4 & Sed 7 & Batch & 0.23 & & 1-2 wks* & Trace* & $20 *$ & $(f, i)$ \\
\hline $\mathrm{Sr}(\mathrm{II})$ & T-106 Soln. 5 & Sed 7 & Batch & 20.2 & & 1-2 wks* & Trace* & $20 *$ & $(f, i)$ \\
\hline $\mathrm{Sr}(\mathrm{II})$ & N-75 GW & N-94A(10-12) & Batch & 27 & & 3 days & Trace & 30 & (j) \\
\hline $\mathrm{Sr}(\mathrm{II})$ & N-75 GW & N-94A(10-12) & Batch & 32 & 1 & 10 days & Trace & 30 & (j) \\
\hline $\mathrm{Sr}(\mathrm{II})$ & N-75 GW & N-94A(10-12) & Batch & 30.3 & 0.6 & 31 days & Trace & 30 & (j) \\
\hline $\mathrm{Sr}(\mathrm{II})$ & N-75 GW & N-94A(15-17) & Batch & 24 & & 3 days & Trace & 30 & (j) \\
\hline $\operatorname{Sr}(\mathrm{II})$ & $\mathrm{N}-75 \mathrm{GW}$ & N-94A(15-17) & Batch & 28 & 4 & 10 days & Trace & 30 & (j) \\
\hline
\end{tabular}




\begin{tabular}{|c|c|c|c|c|c|c|c|c|c|}
\hline Species & $\begin{array}{l}\text { Aqueous } \\
\text { Phase }\end{array}$ & Solid Phase & Method & $\begin{array}{c}\mathrm{Kd} \\
(\mathrm{mL} / \mathrm{g})\end{array}$ & Std. Dev. & $\begin{array}{c}\text { Equil. } \\
\text { Time }\end{array}$ & $\begin{array}{c}\text { In. Soln. } \\
\text { Conc. }\end{array}$ & $\begin{array}{l}\text { Solution/ } \\
\text { Solid } \\
(\mathrm{mL} / \mathrm{g})\end{array}$ & Reference \\
\hline $\mathrm{Sr}(\mathrm{II})$ & N-75 GW & N-94A(15-17) & Batch & 28 & 2 & 31 days & Trace & 30 & (j) \\
\hline $\operatorname{Sr}(\mathrm{II})$ & N-75 GW & N-94A(45) & Batch & 273 & & 3 days & Trace & 30 & (j) \\
\hline $\operatorname{Sr}(\mathrm{II})$ & N-75 GW & N-94A(45) & Batch & 266 & 6 & 10 days & Trace & 30 & (j) \\
\hline $\mathrm{Sr}(\mathrm{II})$ & N-75 GW & N-94A(45) & Batch & 252 & 18 & 31 days & Trace & 30 & (j) \\
\hline $\mathrm{Sr}(\mathrm{II})$ & N-75 GW & N-95A(10) & Batch & 41 & & 3 days & Trace & 30 & (j) \\
\hline $\mathrm{Sr}(\mathrm{II})$ & N-75 GW & N-95A(10) & Batch & 47 & 5 & 10 days & Trace & 30 & (j) \\
\hline $\operatorname{Sr}(\mathrm{II})$ & N-75 GW & N-95A(10) & Batch & 49 & 4 & 31 days & Trace & 30 & (j) \\
\hline $\mathrm{Sr}(\mathrm{II})$ & N-75 GW & N-95A(20) & Batch & 25 & & 3 days & Trace & 30 & (j) \\
\hline $\mathrm{Sr}(\mathrm{II})$ & N-75 GW & N-95A(20) & Batch & 36 & 15 & 10 days & Trace & 30 & (j) \\
\hline $\mathrm{Sr}(\mathrm{II})$ & N-75 GW & N-95A(20) & Batch & 26 & 3 & 31 days & Trace & 30 & (j) \\
\hline $\mathrm{Sr}(\mathrm{II})$ & N-75 GW & N-95A(39) & Batch & 124 & & 3 days & Trace & 30 & (j) \\
\hline $\operatorname{Sr}(\mathrm{II})$ & N-75 GW & N-95A(39) & Batch & 131 & 16 & 10 days & Trace & 30 & (j) \\
\hline $\operatorname{Sr}(\mathrm{II})$ & N-75 GW & N-95A(39) & Batch & 124 & 1 & 31 days & Trace & 30 & (j) \\
\hline $\mathrm{Sr}(\mathrm{II})$ & N-75 GW & N-96A(10-12) & Batch & 33 & & 3 days & Trace & 30 & (j) \\
\hline $\mathrm{Sr}(\mathrm{II})$ & N-75 GW & N-96A(10-12) & Batch & 35 & 2 & 10 days & Trace & 30 & (j) \\
\hline $\operatorname{Sr}(\mathrm{II})$ & N-75 GW & N-96A(10-12) & Batch & 36 & 3 & 31 days & Trace & 30 & (j) \\
\hline $\operatorname{Sr}(\mathrm{II})$ & N-75 GW & N-96A(20-22) & Batch & 17 & & 3 days & Trace & 30 & (j) \\
\hline $\operatorname{Sr}(\mathrm{II})$ & N-75 GW & N-96A(20-22) & Batch & 21 & 3 & 10 days & Trace & 30 & (j) \\
\hline $\operatorname{Sr}(\mathrm{II})$ & N-75 GW & N-96A(20-22) & Batch & 20 & 0 & 31 days & Trace & 30 & (j) \\
\hline $\mathrm{Sr}(\mathrm{II})$ & N-75 GW & N-96A(45-46) & Batch & 63 & & 3 days & Trace & 30 & (j) \\
\hline $\operatorname{Sr}(\mathrm{II})$ & N-75 GW & $\mathrm{N}-96 \mathrm{~A}(45-46)$ & Batch & 49 & 14 & 10 days & Trace & 30 & (j) \\
\hline $\operatorname{Sr}(\mathrm{II})$ & N-75 GW & N-96A(45-46) & Batch & 49 & 17 & 31 days & Trace & 30 & (j) \\
\hline \multicolumn{10}{|c|}{$\begin{array}{l}\text { (a) Serne et al. 1993. } \\
\text { (b) Gee and Campbell } 1980 . \\
\text { (c) Kaplan et al. 1998b. } \\
\text { (d) Lindenmeier et al. } 1995 . \\
\text { (e) Delegard and Barney } 1983 . \\
\text { (f) } \text { Serne et al. 1998. } \\
\text { (g) Hajek and Ames } 1966 . \\
\text { (h) } \text { Routson 1973. } \\
\text { (i) } \text { Ames and Rai 1978. } \\
\text { (j) Serne and LeGore } 1996 . \\
\text { *Data is best estimate. }\end{array}$} \\
\hline
\end{tabular}


Table 10. Contaminant Distribution Coefficient Data for Chromium

\begin{tabular}{|c|c|c|c|c|c|c|c|c|c|}
\hline Species & $\begin{array}{c}\text { Aqueous } \\
\text { Phase }\end{array}$ & Solid Phase & Method & $\begin{array}{c}\mathrm{Kd} \\
(\mathrm{mL} / \mathrm{g})\end{array}$ & Std. Dev. & $\begin{array}{c}\text { Equil. } \\
\text { Time }\end{array}$ & $\begin{array}{c}\text { In. Soln. } \\
\text { Conc. }\end{array}$ & $\begin{array}{l}\text { Solution/ } \\
\text { Solid } \\
(\mathrm{mL} / \mathrm{g})\end{array}$ & Reference \\
\hline $\mathrm{Cr}(\mathrm{VI})$ & Hanford GW & CGS-1 & Batch & 0.5 & 0.6 & 4 days & $1.92 \mathrm{E}-06$ & 30 & (a) \\
\hline $\mathrm{Cr}(\mathrm{VI})$ & $\begin{array}{l}\text { Acid Proc } \\
\text { Waste }\end{array}$ & CGS-1 & Batch & 7.5 & 2.3 & 4 days & $1.92 \mathrm{E}-06$ & 30 & (a) \\
\hline $\mathrm{Cr}(\mathrm{VI})$ & Hanford GW & CGS-1 & Batch & -0.5 & 0.6 & 14 days & $1.92 \mathrm{E}-06$ & 30 & (a) \\
\hline $\mathrm{Cr}(\mathrm{VI})$ & $\begin{array}{l}\text { Acid Proc } \\
\text { Waste }\end{array}$ & CGS-1 & Batch & 13.3 & 4.4 & 14 days & $1.92 \mathrm{E}-06$ & 30 & (a) \\
\hline $\mathrm{Cr}(\mathrm{VI})$ & Hanford GW & CGS-1 & Batch & 0.3 & 1.3 & 35 days & $1.92 \mathrm{E}-06$ & 30 & (a) \\
\hline $\mathrm{Cr}(\mathrm{VI})$ & $\begin{array}{l}\text { Acid Proc } \\
\text { Waste }\end{array}$ & CGS-1 & Batch & 16.3 & 10.3 & 35 days & $1.92 \mathrm{E}-06$ & 30 & (a) \\
\hline $\mathrm{Cr}(\mathrm{VI})$ & Hanford GW & TBS-1 & Batch & 1.02 & 0.79 & 4 days & $1.92 \mathrm{E}-06$ & 30 & (a) \\
\hline $\mathrm{Cr}(\mathrm{VI})$ & $\begin{array}{l}\text { Acid Proc } \\
\text { Waste }\end{array}$ & TBS-1 & Batch & 2.49 & 1.98 & 4 days & $1.92 \mathrm{E}-06$ & 30 & (a) \\
\hline $\mathrm{Cr}(\mathrm{VI})$ & Hanford GW & TBS-1 & Batch & -0.34 & 0.52 & 14 days & $1.92 \mathrm{E}-06$ & 30 & (a) \\
\hline $\mathrm{Cr}(\mathrm{VI})$ & $\begin{array}{l}\text { Acid Proc } \\
\text { Waste }\end{array}$ & TBS-1 & Batch & 13.5 & 8.5 & 14 days & $1.92 \mathrm{E}-06$ & 30 & (a) \\
\hline $\mathrm{Cr}(\mathrm{VI})$ & Hanford GW & TBS-1 & Batch & -0.88 & 0.19 & 35 days & $1.92 \mathrm{E}-06$ & 30 & (a) \\
\hline $\mathrm{Cr}(\mathrm{VI})$ & $\begin{array}{l}\text { Acid Proc } \\
\text { Waste }\end{array}$ & TBS-1 & Batch & 22.1 & 6 & 35 days & $1.92 \mathrm{E}-06$ & 30 & (a) \\
\hline $\mathrm{Cr}(\mathrm{VI})$ & Hanford GW & Trench-8 & Batch & -1.3 & 1.3 & 5 days & $1.92 \mathrm{E}-06$ & 30 & (a) \\
\hline $\mathrm{Cr}(\mathrm{VI})$ & Hanford GW & Trench-8 & Batch & -3.4 & 6.6 & 44 days & $1.92 \mathrm{E}-06$ & 30 & (a) \\
\hline $\mathrm{Cr}(\mathrm{VI})$ & 199-D5-42 & 100-D & Batch & 0.3 & 0.1 & 4 days & $1.92 \mathrm{E}-06$ & 4 & (b) \\
\hline $\mathrm{Cr}(\mathrm{VI})$ & 199-D5-42 & $100-\mathrm{D}$ & Batch & 0.0 & 0.0 & 4 days & 1.92E-05 & 4 & (b) \\
\hline $\mathrm{Cr}(\mathrm{VI})$ & 199-D5-42 & $100-\mathrm{D}$ & Batch & 0.0 & 0.05 & 4 days & $1.92 \mathrm{E}-04$ & 4 & (b) \\
\hline $\mathrm{Cr}(\mathrm{VI})$ & 199-D5-42 & $100-\mathrm{D}$ & Batch & 0.2 & 0.1 & 14 days & 1.92E-06 & 4 & (b) \\
\hline $\mathrm{Cr}(\mathrm{VI})$ & 199-D5-42 & $100-\mathrm{D}$ & Batch & 0.0 & 0.0 & 14 days & 1.92E-05 & 4 & (b) \\
\hline $\mathrm{Cr}(\mathrm{VI})$ & 199-D5-42 & $100-\mathrm{D}$ & Batch & 0.0 & 0.0 & 14 days & 1.92E-04 & 4 & (b) \\
\hline \multicolumn{10}{|c|}{$\begin{array}{l}\text { (a) Serne et al. } 1993 . \\
\text { (b) Serne, R. J. and K. E. Parker. 1999. Estimate of Distribution Coefficients and Leachability of Hexavalent Chromium in } \\
\text { 100--D Area Hanford Formation Sediments. Bechtel Hanford, Inc., Richland, Washington (unpublished report). }\end{array}$} \\
\hline
\end{tabular}


Table 11. Contaminant Distribution Coefficient Data for Iodine

\begin{tabular}{|c|c|c|c|c|c|c|c|c|c|}
\hline Species & $\begin{array}{c}\text { Aqueous } \\
\text { Phase } \\
\end{array}$ & Solid Phase & Method & $\begin{array}{c}\mathrm{Kd} \\
(\mathrm{mL} / \mathrm{g}) \\
\end{array}$ & Std. Dev. & $\begin{array}{c}\text { Equil. Time } \\
\text { (or Res. } \\
\text { Time) } \\
\end{array}$ & $\begin{array}{c}\text { In. Soln. } \\
\text { Conc. }\end{array}$ & $\begin{array}{c}\text { Solution/ } \\
\text { Solid } \\
(\mathrm{mL} / \mathrm{g}) \\
\end{array}$ & Reference \\
\hline $\mathrm{I}(\mathrm{I})$ & Hanford GW & CGS-1 & Batch & 0.2 & 0.3 & 4 days & Trace & 30 & (a) \\
\hline $\mathrm{I}(\mathrm{I})$ & $\begin{array}{l}\text { Acid Proc } \\
\text { Waste }\end{array}$ & CGS-1 & Batch & 0.5 & 1.2 & 4 days & Trace & 30 & (a) \\
\hline $\mathrm{I}(\mathrm{I})$ & Hanford GW & CGS-1 & Batch & 0.9 & 0.4 & 14 days & Trace & 30 & (a) \\
\hline I(I) & $\begin{array}{l}\text { Acid Proc } \\
\text { Waste }\end{array}$ & CGS-1 & Batch & 4.4 & 0.7 & 14 days & Trace & 30 & (a) \\
\hline $\mathrm{I}(\mathrm{I})$ & Hanford GW & CGS-1 & Batch & 1.2 & 0.4 & 35 days & Trace & 30 & (a) \\
\hline I(I) & $\begin{array}{l}\text { Acid Proc } \\
\text { Waste }\end{array}$ & CGS-1 & Batch & 15.9 & 1.6 & 35 days & Trace & 30 & (a) \\
\hline & & & & & & & & & \\
\hline $\mathrm{I}(\mathrm{I})$ & Hanford GW & TBS-1 & Batch & 0.4 & 0.6 & 4 days & Trace & 30 & (a) \\
\hline I(I) & $\begin{array}{l}\text { Acid Proc } \\
\text { Waste }\end{array}$ & TBS-1 & Batch & 4.7 & 0.2 & 4 days & Trace & 30 & (a) \\
\hline $\mathrm{I}(\mathrm{I})$ & Hanford GW & TBS-1 & Batch & 1.2 & 0.9 & 14 days & Trace & 30 & (a) \\
\hline $\mathrm{I}(\mathrm{I})$ & $\begin{array}{l}\text { Acid Proc } \\
\text { Waste }\end{array}$ & TBS-1 & Batch & 5.5 & 0.2 & 14 days & Trace & 30 & (a) \\
\hline $\mathrm{I}(\mathrm{I})$ & Hanford GW & TBS-1 & Batch & 2.6 & 0.4 & 35 days & Trace & 30 & (a) \\
\hline $\mathrm{I}(\mathrm{I})$ & $\begin{array}{l}\text { Acid Proc } \\
\text { Waste }\end{array}$ & TBS-1 & Batch & 5.8 & 0.6 & 35 days & Trace & 30 & (a) \\
\hline $\mathrm{I}(\mathrm{I})$ & Hanford GW & Trench-8 & Batch & 0.9 & 0.2 & 5 days & Trace & 30 & (a) \\
\hline I(I) & Hanford GW & Trench-8 & Batch & 1.5 & 2.4 & 44 days & Trace & 30 & (a) \\
\hline & & & & & & & & & \\
\hline $\mathrm{I}(\mathrm{I})$ & Solution 1 & Soil B & Batch & 7 & 2 & 7 days & Trace & 30 & (b) \\
\hline $\mathrm{I}(\mathrm{I})$ & Solution 2 & Soil B & Batch & 8 & 1 & 7 days & Trace & 30 & (b) \\
\hline I(I) & Solution 3 & Soil B & Batch & 11 & 3 & 7 days & Trace & 30 & (b) \\
\hline $\mathrm{I}(\mathrm{I})$ & Solution 4 & Soil B & Batch & 15 & 5 & 7 days & Trace & 30 & (b) \\
\hline I(I) & Solution 5 & Soil B & Batch & 10 & 4 & 7 days & Trace & 30 & (b) \\
\hline $\mathrm{I}(\mathrm{I})$ & Solution 6 & Soil B & Batch & 4 & 2 & 7 days & Trace & 30 & (b) \\
\hline I(I) & Solution L & Soil B & Batch & 18 & 22 & 7 days & Trace & 30 & (b) \\
\hline $\mathrm{I}(\mathrm{I})$ & Solution H & Soil B & Batch & 4 & 6 & 7 days & Trace & 30 & (b) \\
\hline $\mathrm{I}(\mathrm{I})$ & Solution H & Soil B & Batch & 0.04 & & 4 days & Trace & 30 & (b) \\
\hline I(I) & Solution L & Soil A & Batch & 0.06 & & 4 days & Trace & 30 & (b) \\
\hline & & & & & & & & & \\
\hline $\mathrm{I}(\mathrm{I})$ & Hanford GW2 & B850007A & Batch & 0 & 0.01 & 14 days & Trace & 2 & (c) \\
\hline I(I) & Hanford GW2 & B850010A & Batch & -0.01 & 0.02 & 14 days & Trace & 2 & (c) \\
\hline $\mathrm{I}(\mathrm{I})$ & Hanford GW2 & B850012A & Batch & 0 & 0.03 & 14 days & Trace & 2 & (c) \\
\hline $\mathrm{I}(\mathrm{I})$ & Hanford GW2 & B850014A & Batch & -0.03 & 0.02 & 14 days & Trace & 2 & (c) \\
\hline $\mathrm{I}(\mathrm{I})$ & Hanford GW2 & B850015A & Batch & -0.03 & 0.03 & 14 days & Trace & 2 & (c) \\
\hline I(I) & Hanford GW2 & B850016A & Batch & 0.06 & 0.17 & 14 days & Trace & 2 & (c) \\
\hline I(I) & Hanford GW2 & B850017A & Batch & 0 & 0.02 & 14 days & Trace & 2 & (c) \\
\hline
\end{tabular}




\begin{tabular}{|c|c|c|c|c|c|c|c|c|c|}
\hline Species & $\begin{array}{c}\text { Aqueous } \\
\text { Phase } \\
\end{array}$ & Solid Phase & Method & $\begin{array}{c}\mathrm{Kd} \\
(\mathrm{mL} / \mathrm{g}) \\
\end{array}$ & Std. Dev. & $\begin{array}{c}\text { Equil. Time } \\
\text { (or Res. } \\
\text { Time) } \\
\end{array}$ & $\begin{array}{c}\text { In. Soln. } \\
\text { Conc. }\end{array}$ & $\begin{array}{l}\text { Solution/ } \\
\text { Solid } \\
(\mathrm{mL} / \mathrm{g}) \\
\end{array}$ & Reference \\
\hline I(C) & Hanford GW2 & B850019A & Batch & -0.01 & 0.01 & 14 days & Trace & 2 & (c) \\
\hline $\mathrm{I}(\mathrm{I})$ & Hanford GW2 & B850020A & Batch & -0.01 & 0.02 & 14 days & Trace & 2 & (c) \\
\hline I(C) & Hanford GW2 & B850021A & Batch & -0.01 & 0.01 & 14 days & Trace & 2 & (c) \\
\hline $\mathrm{I}(\mathrm{I})$ & Hanford GW2 & B850022A & Batch & 0.12 & 0.04 & 14 days & Trace & 2 & (c) \\
\hline I(C) & Hanford GW2 & B850023A & Batch & 0.13 & 0.06 & 14 days & Trace & 2 & (c) \\
\hline I('I) & Hanford GW2 & B850024A & Batch & 0.02 & 0.03 & 14 days & Trace & 2 & (c) \\
\hline $\mathrm{I}(\mathrm{I})$ & Hanford GW2 & B850025A & Batch & 0.09 & 0.05 & 14 days & Trace & 2 & (c) \\
\hline $\mathrm{I}(\mathrm{I})$ & Hanford GW2 & B850027A & Batch & 0.06 & 0.05 & 14 days & Trace & 2 & (c) \\
\hline I(I) & Hanford GW2 & B850029A & Batch & 0.23 & 0.06 & 14 days & Trace & 2 & (c) \\
\hline $\mathrm{I}(\mathrm{I})$ & Hanford GW2 & B850031A & Batch & 0.04 & 0.07 & 14 days & Trace & 2 & (c) \\
\hline $\mathrm{I}(\mathrm{I})$ & Hanford GW2 & B850032A & Batch & -0.01 & 0.01 & 14 days & Trace & 2 & (c) \\
\hline $\mathrm{I}(\mathrm{I})$ & Hanford GW2 & B850034A & Batch & 0.08 & 0.08 & 14 days & Trace & 2 & (c) \\
\hline $\mathrm{I}(\mathrm{I})$ & Hanford GW2 & B850035A & Batch & 0.01 & 0.02 & 14 days & Trace & 2 & (c) \\
\hline $\mathrm{I}(\mathrm{I})$ & GW-pH 8.1 & Trench AE-3 & Batch & 0.22 & 0.01 & 14 days & Trace & 2 & (d) \\
\hline I(I) & GW-pH 9.9 & Trench AE-3 & Batch & 0.01 & 0.01 & 14 days & Trace & 2 & (d) \\
\hline $\mathrm{I}(\mathrm{I})$ & GW-pH 10.2 & Trench AE-3 & Batch & -0.02 & 0.02 & 14 days & Trace & 2 & (d) \\
\hline $\mathrm{I}(\mathrm{I})$ & GW-pH 11.0 & \begin{tabular}{|l|} 
Trench AE-3 \\
\end{tabular} & Batch & -0.04 & 0.02 & 14 days & Trace & 2 & (d) \\
\hline $\mathrm{I}(\mathrm{I})$ & GW-pH 11.9 & Trench AE-3 & Batch & 0.01 & 0.01 & 14 days & Trace & 2 & (d) \\
\hline & & & & & & & & & \\
\hline $\mathrm{I}(\mathrm{I})$ & HGW4 & Trench 94 & Batch & 0.19 & 0.1 & 7 & Trace & 2 & (d) \\
\hline $\mathrm{I}(\mathrm{I})$ & HGW4 & Trench 94 & Batch & 2.26 & 1.18 & 199 & Trace & 2 & (d) \\
\hline I(I) & HGW4 & Trench 94 & Batch & 3.31 & 0.11 & 261 & Trace & 2 & (d) \\
\hline $\mathrm{I}(\mathrm{I})$ & HGW4 & Trench 94 & Batch & 9.8 & 0.73 & 331 & Trace & 2 & (d) \\
\hline $\mathrm{I}(\mathrm{I})$ & HGW4 & Trench AE-3 & Batch & 2.09 & 1.14 & 7 & Trace & 2 & (d) \\
\hline $\mathrm{I}(\mathrm{I})$ & HGW4 & Trench AE-3 & Batch & 2.13 & & 199 & Trace & 2 & (d) \\
\hline $\mathrm{I}(\mathrm{I})$ & HGW4 & Trench AE-3 & Batch & 3.26 & 1.96 & 261 & Trace & 2 & (d) \\
\hline $\mathrm{I}(\mathrm{I})$ & HGW4 & Trench AE-3 & Batch & 4.72 & 3.03 & 331 & Trace & 2 & (d) \\
\hline $\mathrm{I}(\mathrm{I})$ & HGW4 & TSB-1 & Batch & 1.11 & 0.08 & 7 & Trace & 2 & (d) \\
\hline I('I) & HGW4 & TSB-1 & Batch & 0.04 & 0.1 & 199 & Trace & 2 & (d) \\
\hline $\mathrm{I}(\mathrm{I})$ & HGW4 & TSB-1 & Batch & 10.44 & 1.49 & 261 & Trace & 2 & (d) \\
\hline $\mathrm{I}(\mathrm{I})$ & HGW4 & TSB-1 & Batch & 6.86 & 1.38 & 331 & Trace & 2 & (d) \\
\hline \multicolumn{10}{|c|}{$\begin{array}{l}\text { (a) Serne et al. } 1993 . \\
\text { (b) Gee and Campbell } 1980 . \\
\text { (c) Kaplan et al. 1998b. } \\
\text { (d) Kaplan et al. } 1996 .\end{array}$} \\
\hline
\end{tabular}


Table 12. Contaminant Distribution Coefficient Data for Nitrate

\begin{tabular}{|c|c|c|c|c|c|c|c|c|c|}
\hline Species & $\begin{array}{l}\text { Aqueous } \\
\text { Phase }\end{array}$ & $\begin{array}{l}\text { Solid } \\
\text { Phase }\end{array}$ & Method & $\begin{array}{c}\mathrm{Kd} \\
(\mathrm{mL} / \mathrm{g})\end{array}$ & $\begin{array}{l}\text { Std. } \\
\text { Dev. }\end{array}$ & Equil. Time & $\begin{array}{c}\text { In. Soln. } \\
\text { Conc. } \\
\text { (mg/L) }\end{array}$ & $\begin{array}{l}\text { Solution/ } \\
\text { Solid } \\
(\mathrm{mL} / \mathrm{g})\end{array}$ & Reference \\
\hline $\mathrm{NO}_{3}{ }^{-}$ & Hanford GW & CGS-1 & Batch & -1.1 & 3.8 & 4 days & 10 & 30 & (a) \\
\hline $\mathrm{NO}_{3}^{-}$ & $\begin{array}{l}\text { Acid Proc } \\
\text { Waste }\end{array}$ & CGS-1 & Batch & -0.5 & 0.8 & 4 days & 260 & 30 & (a) \\
\hline $\mathrm{NO}_{3}^{-}$ & Hanford GW & CGS-1 & Batch & 5.9 & 8.7 & 14 days & 10 & 30 & (a) \\
\hline $\mathrm{NO}_{3}^{-}$ & $\begin{array}{l}\text { Acid Proc } \\
\text { Waste }\end{array}$ & CGS-1 & Batch & -1.9 & 2.2 & 14 days & 260 & 30 & (a) \\
\hline $\mathrm{NO}_{3}^{-}$ & Hanford GW & CGS-1 & Batch & -1.3 & 1.1 & 35 days & 10 & 30 & (a) \\
\hline $\mathrm{NO}_{3}^{-}$ & $\begin{array}{l}\text { Acid Proc } \\
\text { Waste }\end{array}$ & CGS-1 & Batch & -1.9 & 0.6 & 35 days & 260 & 30 & (a) \\
\hline $\mathrm{NO}_{3}^{-}$ & Hanford GW & TBS-1 & Batch & -27.2 & 0.53 & 4 days & 10 & 30 & (a) \\
\hline $\mathrm{NO}_{3}^{-}$ & $\begin{array}{l}\text { Acid Proc } \\
\text { Waste }\end{array}$ & TBS-1 & Batch & 3.4 & 0.7 & 4 days & 260 & 30 & (a) \\
\hline $\mathrm{NO}_{3}^{-}$ & Hanford GW & TBS-1 & Batch & -27 & 0.75 & 14 days & 10 & 30 & (a) \\
\hline $\mathrm{NO}_{3}^{-}$ & $\begin{array}{l}\text { Acid Proc } \\
\text { Waste }\end{array}$ & TBS-1 & Batch & 0.6 & 2.4 & 14 days & 260 & 30 & (a) \\
\hline $\mathrm{NO}_{3}^{-}$ & Hanford GW & TBS-1 & Batch & -26.6 & 0.5 & 35 days & 10 & 30 & (a) \\
\hline $\mathrm{NO}_{3}^{-}$ & $\begin{array}{l}\text { Acid Proc } \\
\text { Waste }\end{array}$ & TBS-1 & Batch & -4.8 & 1.8 & 35 days & 260 & 30 & (a) \\
\hline
\end{tabular}


Table 13. Contaminant Distribution Coefficient Data for Neptunium

\begin{tabular}{|c|c|c|c|c|c|c|c|c|c|}
\hline Species & $\begin{array}{c}\text { Aqueous } \\
\text { Phase }\end{array}$ & Solid Phase & Method & $\begin{array}{c}\mathrm{Kd} \\
(\mathrm{mL} / \mathrm{g})\end{array}$ & Std. Dev. & $\begin{array}{l}\text { Equil. } \\
\text { Time }\end{array}$ & $\begin{array}{c}\text { In. Soln. } \\
\text { Conc. }\end{array}$ & $\begin{array}{l}\text { Solution/ } \\
\text { Solid } \\
(\mathrm{mL} / \mathrm{g})\end{array}$ & Reference \\
\hline $\mathrm{Np}(\mathrm{V})$ & Hanford GW & Trench- 8 & Batch & $1.35 \mathrm{E}+01$ & $3.00 \mathrm{E}+00$ & 5 days & Trace & 30 & (a) \\
\hline $\mathrm{Np}(\mathrm{V})$ & Hanford GW & Trench- 8 & Batch & $2.91 \mathrm{E}+01$ & $3.60 \mathrm{E}+00$ & 44 days & Trace & 30 & (a) \\
\hline $\mathrm{Np}(\mathrm{V})$ & $\begin{array}{l}0.015 \mathrm{M} \\
\mathrm{NaNO}_{3} \\
\end{array}$ & Burbank Sand & Batch & 3.9 & 0.12 & 1 day & Trace & 2.5 & $(b, c)$ \\
\hline $\mathrm{Np}(\mathrm{V})$ & $\begin{array}{l}0.030 \mathrm{M} \\
\mathrm{NaNO}_{3} \\
\end{array}$ & Burbank Sand & Batch & 3.51 & 0.19 & 1 day & Trace & 2.5 & $(b, c)$ \\
\hline $\mathrm{Np}(\mathrm{V})$ & $0.30 \mathrm{M} \mathrm{NaNO}_{3}$ & Burbank Sand & Batch & 3.28 & 0.13 & 1 day & Trace & 2.5 & $(b, c)$ \\
\hline $\mathrm{Np}(\mathrm{V})$ & $0.75 \mathrm{M} \mathrm{NaNO}_{3}$ & Burbank Sand & Batch & 3.28 & 0.35 & 1 day & Trace & 2.5 & $(b, c)$ \\
\hline $\mathrm{Np}(\mathrm{V})$ & $3.00 \mathrm{M} \mathrm{NaNO}_{3}$ & Burbank Sand & Batch & 3.19 & 0.22 & 1 day & Trace & 2.5 & $(b, c)$ \\
\hline $\mathrm{Np}(\mathrm{V})$ & $\begin{array}{l}0.002 \mathrm{M} \\
\mathrm{Ca}\left(\mathrm{NO}_{3}\right)_{2}\end{array}$ & Burbank Sand & Batch & 2.37 & 0.04 & 1 day & Trace & 2.5 & $(b, c)$ \\
\hline $\mathrm{Np}(\mathrm{V})$ & $\begin{array}{l}0.02 \mathrm{M} \\
\mathrm{Ca}\left(\mathrm{NO}_{3}\right)_{2} \\
\end{array}$ & Burbank Sand & Batch & 0.93 & 0.07 & 1 day & Trace & 2.5 & $(b, c)$ \\
\hline $\mathrm{Np}(\mathrm{V})$ & $\begin{array}{l}0.05 \mathrm{M} \\
\mathrm{Ca}\left(\mathrm{NO}_{3}\right)_{2}\end{array}$ & Burbank Sand & Batch & 0.78 & 0.16 & 1 day & Trace & 2.5 & $(b, c)$ \\
\hline $\mathrm{Np}(\mathrm{V})$ & $\begin{array}{l}0.10 \mathrm{M} \\
\mathrm{Ca}\left(\mathrm{NO}_{3}\right)_{2}\end{array}$ & Burbank Sand & Batch & 0.62 & 0.04 & 1 day & Trace & 2.5 & $(b, c)$ \\
\hline $\mathrm{Np}(\mathrm{V})$ & $\begin{array}{l}0.20 \mathrm{M} \\
\mathrm{Ca}\left(\mathrm{NO}_{3}\right)_{2}\end{array}$ & Burbank Sand & Batch & 0.36 & 0.07 & 1 day & Trace & 2.5 & $(b, c)$ \\
\hline $\mathrm{Np}(\mathrm{V})$ & Distilled water & Burbank Sand & Batch & 7.1 & 0.7 & 4 days & Trace & 10 & (d) \\
\hline $\mathrm{Np}(\mathrm{V})$ & Distilled water & Burbank Sand & Batch & 6.3 & 0.7 & 8 days & Trace & 10 & (d) \\
\hline $\mathrm{Np}(\mathrm{V})$ & Distilled water & Burbank Sand & Batch & 7.1 & 0.7 & 16 days & Trace & 10 & (d) \\
\hline $\mathrm{Np}(\mathrm{V})$ & Distilled water & Burbank Sand & Batch & 10.8 & 1.1 & 28 days & Trace & 10 & (d) \\
\hline $\mathrm{Np}(\mathrm{V})$ & Distilled water & Burbank Sand & Batch & 12.4 & 1.3 & 42 days & Trace & 10 & (d) \\
\hline $\mathrm{Np}(\mathrm{V})$ & Distilled water & Burbank Sand & Batch & 45 & 5 & 100 days & Trace & 10 & (d) \\
\hline $\mathrm{Np}(\mathrm{V})$ & Soln 1 & Sed L & Batch & 9.4 & & 7 days & Trace & 5 & (e) \\
\hline $\mathrm{Np}(\mathrm{V})$ & Soln 2 & Sed L & Batch & 1.62 & & 7 days & Trace & 5 & (e) \\
\hline $\mathrm{Np}(\mathrm{V})$ & Soln 3 & Sed L & Batch & 1.75 & & 7 days & Trace & 5 & (e) \\
\hline $\mathrm{Np}(\mathrm{V})$ & Soln 4 & Sed L & Batch & 1.09 & & 7 days & Trace & 5 & (e) \\
\hline $\mathrm{Np}(\mathrm{V})$ & Soln 5 & Sed L & Batch & 2.95 & & 7 days & Trace & 5 & (e) \\
\hline $\mathrm{Np}(\mathrm{V})$ & Soln 6 & Sed L & Batch & 12.43 & & 7 days & Trace & 5 & (e) \\
\hline $\mathrm{Np}(\mathrm{V})$ & Soln 7 & Sed L & Batch & 2.43 & & 7 days & Trace & 5 & (e) \\
\hline $\mathrm{Np}(\mathrm{V})$ & Soln 8 & Sed L & Batch & 1.89 & & 7 days & Trace & 5 & (e) \\
\hline $\mathrm{Np}(\mathrm{V})$ & Soln 9 & Sed L & Batch & 1.76 & & 7 days & Trace & 5 & (e) \\
\hline $\mathrm{Np}(\mathrm{V})$ & Soln 10 & Sed L & Batch & 1.8 & & 7 days & Trace & 5 & (e) \\
\hline
\end{tabular}




\begin{tabular}{|c|c|c|c|c|c|c|c|c|c|}
\hline Species & $\begin{array}{c}\text { Aqueous } \\
\text { Phase }\end{array}$ & Solid Phase & Method & $\begin{array}{c}\mathrm{Kd} \\
(\mathrm{mL} / \mathrm{g})\end{array}$ & Std. Dev. & $\begin{array}{l}\text { Equil. } \\
\text { Time }\end{array}$ & $\begin{array}{c}\text { In. Soln. } \\
\text { Conc. }\end{array}$ & $\begin{array}{l}\text { Solution/ } \\
\text { Solid } \\
(\mathrm{mL} / \mathrm{g})\end{array}$ & Reference \\
\hline $\mathrm{Np}(\mathrm{V})$ & Soln 11 & Sed L & Batch & 6.93 & & 7 days & Trace & 5 & (e) \\
\hline $\mathrm{Np}(\mathrm{V})$ & Soln 12 & Sed L & Batch & 4.14 & & 7 days & Trace & 5 & (e) \\
\hline $\mathrm{Np}(\mathrm{V})$ & Soln 13 & Sed L & Batch & 3.62 & & 7 days & Trace & 5 & (e) \\
\hline $\mathrm{Np}(\mathrm{V})$ & Soln 14 & Sed L & Batch & 92.07 & & 7 days & Trace & 5 & (e) \\
\hline $\mathrm{Np}(\mathrm{V})$ & Soln 15 & Sed L & Batch & 0.79 & & 7 days & Trace & 5 & (e) \\
\hline $\mathrm{Np}(\mathrm{V})$ & Soln 16 & Sed L & Batch & 1.61 & & 7 days & Trace & 5 & (e) \\
\hline $\mathrm{Np}(\mathrm{V})$ & Soln 17 & Sed L & Batch & 0.75 & & 7 days & Trace & 5 & (e) \\
\hline $\mathrm{Np}(\mathrm{V})$ & Soln 18 & Sed L & Batch & 1.22 & & 7 days & Trace & 5 & (e) \\
\hline $\mathrm{Np}(\mathrm{V})$ & Soln 19 & Sed L & Batch & 1.71 & & 7 days & Trace & 5 & (e) \\
\hline $\mathrm{Np}(\mathrm{V})$ & Soln 20 & Sed L & Batch & 296 & & 7 days & Trace & 5 & (e) \\
\hline $\mathrm{Np}(\mathrm{V})$ & Soln 1 & Sed P & Batch & 7.06 & & 7 days & Trace & 5 & (e) \\
\hline $\mathrm{Np}(\mathrm{V})$ & Soln 2 & Sed P & Batch & 0.95 & & 7 days & Trace & 5 & (e) \\
\hline $\mathrm{Np}(\mathrm{V})$ & Soln 3 & Sed P & Batch & 1.12 & & 7 days & Trace & 5 & (e) \\
\hline $\mathrm{Np}(\mathrm{V})$ & Soln 4 & Sed P & Batch & 1.13 & & 7 days & Trace & 5 & (e) \\
\hline $\mathrm{Np}(\mathrm{V})$ & Soln 5 & Sed P & Batch & 2.48 & & 7 days & Trace & 5 & (e) \\
\hline $\mathrm{Np}(\mathrm{V})$ & Soln 6 & Sed P & Batch & 10.5 & & 7 days & Trace & 5 & (e) \\
\hline $\mathrm{Np}(\mathrm{V})$ & Soln 7 & Sed P & Batch & 2.06 & & 7 days & Trace & 5 & (e) \\
\hline $\mathrm{Np}(\mathrm{V})$ & Soln 8 & Sed P & Batch & 2.12 & & 7 days & Trace & 5 & (e) \\
\hline $\mathrm{Np}(\mathrm{V})$ & Soln 9 & Sed P & Batch & 1.83 & & 7 days & Trace & 5 & (e) \\
\hline $\mathrm{Np}(\mathrm{V})$ & Soln 10 & Sed P & Batch & 2.03 & & 7 days & Trace & 5 & (e) \\
\hline $\mathrm{Np}(\mathrm{V})$ & Soln 11 & Sed P & Batch & 5.01 & & 7 days & Trace & 5 & (e) \\
\hline $\mathrm{Np}(\mathrm{V})$ & Soln 12 & Sed P & Batch & 4.34 & & 7 days & Trace & 5 & (e) \\
\hline $\mathrm{Np}(\mathrm{V})$ & Soln 13 & Sed P & Batch & 3.75 & & 7 days & Trace & 5 & (e) \\
\hline $\mathrm{Np}(\mathrm{V})$ & Soln 14 & Sed P & Batch & 75.2 & & 7 days & Trace & 5 & (e) \\
\hline $\mathrm{Np}(\mathrm{V})$ & Soln 15 & Sed P & Batch & 0.64 & & 7 days & Trace & 5 & (e) \\
\hline $\mathrm{Np}(\mathrm{V})$ & Soln 16 & Sed P & Batch & 1.11 & & 7 days & Trace & 5 & (e) \\
\hline $\mathrm{Np}(\mathrm{V})$ & Soln 17 & Sed P & Batch & 0.66 & & 7 days & Trace & 5 & (e) \\
\hline $\mathrm{Np}(\mathrm{V})$ & Soln 18 & Sed P & Batch & 1 & & 7 days & Trace & 5 & (e) \\
\hline $\mathrm{Np}(\mathrm{V})$ & Soln 19 & Sed P & Batch & 1.83 & & 7 days & Trace & 5 & (e) \\
\hline $\mathrm{Np}(\mathrm{V})$ & Soln 20 & Sed P & Batch & 142 & & 7 days & Trace & 5 & (e) \\
\hline $\mathrm{Np}(\mathrm{V})$ & Soln 1 & Sed S & Batch & 32.9 & & 7 days & Trace & 5 & (e) \\
\hline $\mathrm{Np}(\mathrm{V})$ & Soln 2 & Sed S & Batch & 3.6 & & 7 days & Trace & 5 & (e) \\
\hline $\mathrm{Np}(\mathrm{V})$ & Soln 3 & Sed S & Batch & 3.06 & & 7 days & Trace & 5 & (e) \\
\hline $\mathrm{Np}(\mathrm{V})$ & Soln 4 & Sed S & Batch & 2.4 & & 7 days & Trace & 5 & (e) \\
\hline $\mathrm{Np}(\mathrm{V})$ & Soln 5 & Sed S & Batch & 12.8 & & 7 days & Trace & 5 & (e) \\
\hline $\mathrm{Np}(\mathrm{V})$ & Soln 6 & Sed S & Batch & 118 & & 7 days & Trace & 5 & (e) \\
\hline
\end{tabular}




\begin{tabular}{|c|c|c|c|c|c|c|c|c|c|}
\hline Species & $\begin{array}{l}\text { Aqueous } \\
\text { Phase }\end{array}$ & Solid Phase & Method & $\begin{array}{c}\mathrm{Kd} \\
(\mathrm{mL} / \mathrm{g})\end{array}$ & Std. Dev. & $\begin{array}{c}\text { Equil. } \\
\text { Time }\end{array}$ & $\begin{array}{c}\text { In. Soln. } \\
\text { Conc. }\end{array}$ & $\begin{array}{l}\text { Solution/ } \\
\text { Solid } \\
(\mathrm{mL} / \mathrm{g})\end{array}$ & Reference \\
\hline $\mathrm{Np}(\mathrm{V})$ & Soln 7 & Sed S & Batch & 6.17 & & 7 days & Trace & 5 & (e) \\
\hline $\mathrm{Np}(\mathrm{V})$ & Soln 8 & Sed S & Batch & 4.66 & & 7 days & Trace & 5 & (e) \\
\hline $\mathrm{Np}(\mathrm{V})$ & Soln 9 & Sed S & Batch & 4.59 & & 7 days & Trace & 5 & (e) \\
\hline $\mathrm{Np}(\mathrm{V})$ & Soln 10 & Sed S & Batch & 5.42 & & 7 days & Trace & 5 & (e) \\
\hline $\mathrm{Np}(\mathrm{V})$ & Soln 11 & Sed S & Batch & 13.9 & & 7 days & Trace & 5 & (e) \\
\hline $\mathrm{Np}(\mathrm{V})$ & Soln 12 & Sed S & Batch & 13.1 & & 7 days & Trace & 5 & (e) \\
\hline $\mathrm{Np}(\mathrm{V})$ & Soln 13 & Sed S & Batch & 11.9 & & 7 days & Trace & 5 & (e) \\
\hline $\mathrm{Np}(\mathrm{V})$ & Soln 14 & Sed S & Batch & 222 & & 7 days & Trace & 5 & (e) \\
\hline $\mathrm{Np}(\mathrm{V})$ & Soln 15 & Sed S & Batch & 2.93 & & 7 days & Trace & 5 & (e) \\
\hline $\mathrm{Np}(\mathrm{V})$ & Soln 16 & Sed S & Batch & 2.88 & & 7 days & Trace & 5 & (e) \\
\hline $\mathrm{Np}(\mathrm{V})$ & Soln 17 & Sed S & Batch & 2.91 & & 7 days & Trace & 5 & (e) \\
\hline $\mathrm{Np}(\mathrm{V})$ & Soln 18 & Sed S & Batch & 2.05 & & 7 days & Trace & 5 & (e) \\
\hline $\mathrm{Np}(\mathrm{V})$ & Soln 19 & Sed S & Batch & 3.8 & & 7 days & Trace & 5 & (e) \\
\hline $\mathrm{Np}(\mathrm{V})$ & Soln 20 & Sed S & Batch & $>2090$ & & 7 days & Trace & 5 & (e) \\
\hline $\mathrm{Np}(\mathrm{V})$ & HGW4 & Trench 94 & Batch & 14.7 & 1.2 & 7 & Trace & 2 & (f) \\
\hline $\mathrm{Np}(\mathrm{V})$ & HGW4 & Trench 94 & Batch & 19.9 & 0.5 & 77 & Trace & 2 & (f) \\
\hline $\mathrm{Np}(\mathrm{V})$ & HGW4 & Trench AE-3 & Batch & 2.7 & 0.2 & 7 & Trace & 2 & (f) \\
\hline $\mathrm{Np}(\mathrm{V})$ & HGW4 & Trench AE-3 & Batch & 13.5 & 0.5 & 77 & Trace & 2 & (f) \\
\hline $\mathrm{Np}(\mathrm{V})$ & HGW4 & TSB-1 & Batch & 2.2 & 0.4 & 7 & Trace & 2 & (f) \\
\hline $\mathrm{Np}(\mathrm{V})$ & HGW4 & TSB-1 & Batch & 3.6 & 3.7 & 77 & Trace & 2 & (f) \\
\hline \multicolumn{10}{|c|}{\begin{tabular}{|ll} 
(a) & Serne et al. 1993. \\
(b) & Routson et al. 1975. \\
(c) & Routson et al. 1976. \\
(d) & Sheppard et al. 1976. \\
(e) & Delegard and Barney 1983. \\
(f) & Kaplan et al. 1996.
\end{tabular}} \\
\hline
\end{tabular}


Table 14. Contaminant Distribution Coefficient Data for Selenium

\begin{tabular}{|c|c|c|c|c|c|c|c|c|c|}
\hline Species & $\begin{array}{l}\text { Aqueous } \\
\text { Phase }\end{array}$ & Solid Phase & Method & $\begin{array}{c}\mathrm{Kd} \\
(\mathrm{mL} / \mathrm{g})\end{array}$ & Std. Dev. & $\begin{array}{l}\text { Equil. } \\
\text { Time }\end{array}$ & $\begin{array}{l}\text { In. Soln. } \\
\text { Conc. }\end{array}$ & $\begin{array}{l}\text { Solution/ } \\
\text { Solid } \\
(\mathrm{mL} / \mathrm{g})\end{array}$ & Reference \\
\hline $\mathrm{Se}(\mathrm{VI})$ & Hanford GW & CGS-1 & Batch & 0.9 & 1.1 & 4 days & $1.27 \mathrm{E}-06$ & 30 & (a) \\
\hline $\mathrm{Se}(\mathrm{VI})$ & $\begin{array}{l}\text { Acid Proc } \\
\text { Waste }\end{array}$ & CGS-1 & Batch & -1.7 & 1.9 & 4 days & $1.27 \mathrm{E}-06$ & 30 & (a) \\
\hline $\mathrm{Se}(\mathrm{VI})$ & Hanford GW & CGS-1 & Batch & 3.1 & 0.8 & 14 days & $1.27 \mathrm{E}-06$ & 30 & (a) \\
\hline $\mathrm{Se}(\mathrm{VI})$ & $\begin{array}{l}\text { Acid Proc } \\
\text { Waste }\end{array}$ & CGS-1 & Batch & -2.6 & 1.4 & 14 days & $1.27 \mathrm{E}-06$ & 30 & (a) \\
\hline $\mathrm{Se}(\mathrm{VI})$ & Hanford GW & CGS-1 & Batch & -1.6 & 0.03 & 35 days & $1.27 \mathrm{E}-06$ & 30 & (a) \\
\hline $\mathrm{Se}(\mathrm{VI})$ & $\begin{array}{l}\text { Acid Proc } \\
\text { Waste }\end{array}$ & CGS-1 & Batch & 0.4 & 1.7 & 35 days & $1.27 \mathrm{E}-06$ & 30 & (a) \\
\hline $\mathrm{Se}(\mathrm{VI})$ & Hanford GW & TBS-1 & Batch & -3.07 & 0.78 & 4 days & $1.27 \mathrm{E}-06$ & 30 & (a) \\
\hline $\mathrm{Se}(\mathrm{VI})$ & $\begin{array}{l}\text { Acid Proc } \\
\text { Waste }\end{array}$ & TBS-1 & Batch & 4.23 & 0.54 & 4 days & $1.27 \mathrm{E}-06$ & 30 & (a) \\
\hline $\mathrm{Se}(\mathrm{VI})$ & Hanford GW & TBS-1 & Batch & -2.78 & 0.51 & 14 days & $1.27 \mathrm{E}-06$ & 30 & (a) \\
\hline $\mathrm{Se}(\mathrm{VI})$ & $\begin{array}{l}\text { Acid Proc } \\
\text { Waste }\end{array}$ & TBS-1 & Batch & 4.69 & 1.03 & 14 days & $1.27 \mathrm{E}-06$ & 30 & (a) \\
\hline $\mathrm{Se}(\mathrm{VI})$ & Hanford GW & TBS-1 & Batch & -4.48 & 2.05 & 35 days & $1.27 \mathrm{E}-06$ & 30 & (a) \\
\hline $\mathrm{Se}(\mathrm{VI})$ & $\begin{array}{l}\text { Acid Proc } \\
\text { Waste }\end{array}$ & TBS-1 & Batch & 4.87 & 0.53 & 35 days & $1.27 \mathrm{E}-06$ & 30 & (a) \\
\hline $\mathrm{Se}(\mathrm{VI})$ & Hanford GW & Trench-8 & Batch & -0.6 & 1.9 & 5 days & $1.27 \mathrm{E}-06$ & 30 & (a) \\
\hline $\mathrm{Se}(\mathrm{VI})$ & Hanford GW & Trench-8 & Batch & -4.1 & 6.1 & 44 days & $1.27 \mathrm{E}-06$ & 30 & (a) \\
\hline $\mathrm{Se}(\mathrm{VI})$ & Solution 1 & Soil B & Batch & 27 & 2 & 7 days & Trace & 30 & (b) \\
\hline $\mathrm{Se}(\mathrm{VI})$ & Solution 2 & Soil B & Batch & 26 & 2 & 7 days & Trace & 30 & (b) \\
\hline $\mathrm{Se}(\mathrm{VI})$ & Solution 3 & Soil B & Batch & 16 & 3 & 7 days & Trace & 30 & (b) \\
\hline $\mathrm{Se}(\mathrm{VI})$ & Solution 4 & Soil B & Batch & 38 & 12 & 7 days & Trace & 30 & (b) \\
\hline $\mathrm{Se}(\mathrm{VI})$ & Solution 5 & Soil B & Batch & 40 & 2 & 7 days & Trace & 30 & (b) \\
\hline $\mathrm{Se}(\mathrm{VI})$ & Solution 6 & Soil B & Batch & 72 & 10 & 7 days & Trace & 30 & (b) \\
\hline $\mathrm{Se}(\mathrm{VI})$ & Hanford GW2 & B850007A & Batch & 7.77 & 0.08 & 14 days & Trace & 2 & (c) \\
\hline $\mathrm{Se}(\mathrm{VI})$ & Hanford GW2 & B850010A & Batch & 6.8 & 0.54 & 14 days & Trace & 2 & (c) \\
\hline $\mathrm{Se}(\mathrm{VI})$ & Hanford GW2 & B850012A & Batch & 6.22 & 0.38 & 14 days & Trace & 2 & (c) \\
\hline $\mathrm{Se}(\mathrm{VI})$ & Hanford GW2 & B850014A & Batch & 6.79 & 0.32 & 14 days & Trace & 2 & (c) \\
\hline $\mathrm{Se}(\mathrm{VI})$ & Hanford GW2 & B850015A & Batch & 6.24 & 0.28 & 14 days & Trace & 2 & (c) \\
\hline $\mathrm{Se}(\mathrm{VI})$ & Hanford GW2 & B850016A & Batch & 6.85 & 0.58 & 14 days & Trace & 2 & (c) \\
\hline $\mathrm{Se}(\mathrm{VI})$ & Hanford GW2 & B850017A & Batch & 8.64 & 0.38 & 14 days & Trace & 2 & (c) \\
\hline $\mathrm{Se}(\mathrm{VI})$ & Hanford GW2 & B850019A & Batch & 8.93 & 0.05 & 14 days & Trace & 2 & (c) \\
\hline
\end{tabular}




\begin{tabular}{|c|c|c|c|c|c|c|c|c|c|}
\hline Species & $\begin{array}{l}\text { Aqueous } \\
\text { Phase }\end{array}$ & Solid Phase & Method & $\begin{array}{c}\mathrm{Kd} \\
(\mathrm{mL} / \mathrm{g})\end{array}$ & Std. Dev. & $\begin{array}{l}\text { Equil. } \\
\text { Time }\end{array}$ & $\begin{array}{c}\text { In. Soln. } \\
\text { Conc. }\end{array}$ & $\begin{array}{l}\text { Solution/ } \\
\text { Solid } \\
(\mathrm{mL} / \mathrm{g})\end{array}$ & Reference \\
\hline $\mathrm{Se}(\mathrm{VI})$ & Hanford GW2 & B850020A & Batch & 7.55 & 0.21 & 14 days & Trace & 2 & (c) \\
\hline $\mathrm{Se}(\mathrm{VI})$ & Hanford GW2 & B850021A & Batch & 6.57 & 0.47 & 14 days & Trace & 2 & (c) \\
\hline $\mathrm{Se}(\mathrm{VI})$ & Hanford GW2 & B850022A & Batch & 6.74 & 0.44 & 14 days & Trace & 2 & (c) \\
\hline $\mathrm{Se}(\mathrm{VI})$ & Hanford GW2 & B850023A & Batch & 10.4 & 0.55 & 14 days & Trace & 2 & (c) \\
\hline $\mathrm{Se}(\mathrm{VI})$ & Hanford GW2 & B850024A & Batch & 6.09 & 0.24 & 14 days & Trace & 2 & (c) \\
\hline $\mathrm{Se}(\mathrm{VI})$ & Hanford GW2 & B850025A & Batch & 5.28 & 0.09 & 14 days & Trace & 2 & (c) \\
\hline $\mathrm{Se}(\mathrm{VI})$ & Hanford GW2 & B850027A & Batch & 4.75 & 0.44 & 14 days & Trace & 2 & (c) \\
\hline $\mathrm{Se}(\mathrm{VI})$ & Hanford GW2 & B850029A & Batch & 5.67 & 0.24 & 14 days & Trace & 2 & (c) \\
\hline $\mathrm{Se}(\mathrm{VI})$ & Hanford GW2 & B850031A & Batch & 10.85 & 0.31 & 14 days & Trace & 2 & (c) \\
\hline $\mathrm{Se}(\mathrm{VI})$ & Hanford GW2 & B850032A & Batch & 4.4 & 0.17 & 14 days & Trace & 2 & (c) \\
\hline $\mathrm{Se}(\mathrm{VI})$ & Hanford GW2 & B850034A & Batch & 4.23 & 0.15 & 14 days & Trace & 2 & (c) \\
\hline $\mathrm{Se}(\mathrm{VI})$ & Hanford GW2 & B850035A & Batch & 3.75 & 0.17 & 14 days & Trace & 2 & (c) \\
\hline $\mathrm{Se}(\mathrm{VI})$ & GW-IS-0.05 & Trench AE-3 & Batch & 3.31 & 0.57 & 14 days & Trace & 2 & (d) \\
\hline $\mathrm{Se}(\mathrm{VI})$ & GW-IS-0.10 & Trench AE-3 & Batch & 4.49 & 0.19 & 14 days & Trace & 2 & (d) \\
\hline $\mathrm{Se}(\mathrm{VI})$ & GW-IS-0.50 & Trench AE-3 & Batch & 4.47 & 0.06 & 14 days & Trace & 2 & (d) \\
\hline $\mathrm{Se}(\mathrm{VI})$ & GW-IS-1.00 & Trench AE-3 & Batch & 4.11 & 0.06 & 14 days & Trace & 2 & (d) \\
\hline $\mathrm{Se}(\mathrm{VI})$ & GW-pH 8.1 & Trench AE-3 & Batch & 5.78 & 0.28 & 14 days & Trace & 2 & (d) \\
\hline $\mathrm{Se}(\mathrm{VI})$ & GW-pH 9.9 & Trench AE-3 & Batch & 0.29 & 0.03 & 14 days & Trace & 2 & (d) \\
\hline $\mathrm{Se}(\mathrm{VI})$ & GW-pH 10.2 & Trench AE-3 & Batch & 0.19 & 0 & 14 days & Trace & 2 & (d) \\
\hline $\mathrm{Se}(\mathrm{VI})$ & GW-pH 11.0 & Trench AE-3 & Batch & 0.05 & 0.02 & 14 days & Trace & 2 & (d) \\
\hline $\mathrm{Se}(\mathrm{VI})$ & GW-pH 11.9 & Trench AE-3 & Batch & 0.04 & 0 & 14 days & Trace & 2 & (d) \\
\hline \multicolumn{10}{|c|}{\begin{tabular}{|l} 
(a) Serne et al. 1993. \\
(b) Gee and Campbell 1980. \\
(c) Kaplan et al. 1998a. \\
(d) Kaplan et al. 1998b.
\end{tabular}} \\
\hline
\end{tabular}


Table 15. Contaminant Distribution Coefficient Data for Technetium

\begin{tabular}{|c|c|c|c|c|c|c|c|c|c|}
\hline Species & $\begin{array}{l}\text { Aqueous } \\
\text { Phase }\end{array}$ & Solid Phase & Method & $\begin{array}{c}\mathrm{Kd} \\
(\mathrm{mL} / \mathrm{g})\end{array}$ & Std. Dev. & $\begin{array}{l}\text { Equil. } \\
\text { Time }\end{array}$ & $\begin{array}{l}\text { In. Soln. } \\
\text { Conc. }\end{array}$ & $\begin{array}{l}\text { Solution/ } \\
\text { Solid } \\
(\mathrm{mL} / \mathrm{g})\end{array}$ & Reference \\
\hline $\mathrm{Tc}(\mathrm{VII})$ & Hanford GW1 & CGS-1 & Batch & -0.1 & 0.8 & 4 days & Trace & 30 & (a) \\
\hline $\mathrm{Tc}(\mathrm{VII})$ & Hanford GW1 & CGS-1 & Batch & 0.4 & 1.1 & 14 days & Trace & 30 & (a) \\
\hline Tc(VII) & Hanford GW1 & CGS-1 & Batch & 0.1 & 0.2 & 35 days & Trace & 30 & (a) \\
\hline $\mathrm{Tc}(\mathrm{VII})$ & $\begin{array}{l}\text { Acid Proc } \\
\text { Waste }\end{array}$ & CGS-1 & Batch & 0.8 & 0.8 & 4 days & Trace & 30 & (a) \\
\hline $\mathrm{Tc}(\mathrm{VII})$ & $\begin{array}{l}\text { Acid Proc } \\
\text { Waste }\end{array}$ & CGS-1 & Batch & -0.4 & 0.3 & 14 days & Trace & 30 & (a) \\
\hline $\mathrm{Tc}(\mathrm{VII})$ & $\begin{array}{l}\text { Acid Proc } \\
\text { Waste }\end{array}$ & CGS-1 & Batch & -0.3 & 0.1 & 35 days & Trace & 30 & (a) \\
\hline $\mathrm{Tc}(\mathrm{VII})$ & Hanford GW1 & TBS-1 & Batch & 0.2 & 0.8 & 4 days & Trace & 30 & (a) \\
\hline $\mathrm{Tc}(\mathrm{VII})$ & Hanford GW1 & TBS-1 & Batch & 0.1 & 0.2 & 14 days & Trace & 30 & (a) \\
\hline $\mathrm{Tc}(\mathrm{VII})$ & Hanford GW1 & TBS-1 & Batch & 0.1 & 0.8 & 35 days & Trace & 30 & (a) \\
\hline $\mathrm{Tc}(\mathrm{VII})$ & $\begin{array}{l}\text { Acid Proc } \\
\text { Waste }\end{array}$ & TBS-1 & Batch & 0.2 & 0.8 & 4 days & Trace & 30 & (a) \\
\hline $\mathrm{Tc}(\mathrm{VII})$ & $\begin{array}{l}\text { Acid Proc } \\
\text { Waste }\end{array}$ & TBS-1 & Batch & -0.2 & 0.5 & 14 days & Trace & 30 & (a) \\
\hline $\mathrm{Tc}(\mathrm{VII})$ & $\begin{array}{l}\text { Acid Proc } \\
\text { Waste }\end{array}$ & TBS-1 & Batch & 0.3 & 1.2 & 35 days & Trace & 30 & (a) \\
\hline $\mathrm{Tc}(\mathrm{VII})$ & Hanford GW1 & Trench- 8 & Batch & -0.1 & 0.2 & 5 days & Trace & 30 & (a) \\
\hline $\mathrm{Tc}(\mathrm{VII})$ & Hanford GW1 & Trench-8 & Batch & 0.2 & 0.7 & 44 days & Trace & 30 & (a) \\
\hline $\mathrm{Tc}(\mathrm{VII})$ & Solution 1 & Soil A & Batch & -0.38 & 1.51 & 8.5 days & Trace & 30 & (b) \\
\hline $\mathrm{Tc}(\mathrm{VII})$ & Solution 2 & Soil A & Batch & -0.88 & 0.52 & 8.5 days & Trace & 30 & (b) \\
\hline $\mathrm{Tc}(\mathrm{VII})$ & Solution 3 & Soil A & Batch & -1.36 & 0.71 & 8.5 days & Trace & 30 & (b) \\
\hline $\mathrm{Tc}(\mathrm{VII})$ & Solution 4 & Soil A & Batch & 0.41 & 0.34 & 8.5 days & Trace & 30 & (b) \\
\hline $\mathrm{Tc}(\mathrm{VII})$ & Solution 5 & Soil A & Batch & -2.34 & 0.98 & 8.5 days & Trace & 30 & (b) \\
\hline $\mathrm{Tc}(\mathrm{VII})$ & Solution 6 & Soil A & Batch & -0.81 & 0.39 & 8.5 days & Trace & 30 & (b) \\
\hline $\mathrm{Tc}(\mathrm{VII})$ & Solution 1 & Soil A & Batch & -2.77 & 0.23 & 21.5 days & Trace & 30 & (b) \\
\hline $\mathrm{Tc}(\mathrm{VII})$ & Solution 2 & Soil A & Batch & -1.13 & 2.38 & 21.5 days & Trace & 30 & (b) \\
\hline $\mathrm{Tc}(\mathrm{VII})$ & Solution 3 & Soil A & Batch & -0.04 & 0.21 & 21.5 days & Trace & 30 & (b) \\
\hline $\mathrm{Tc}(\mathrm{VII})$ & Solution 4 & Soil A & Batch & 0.57 & 0.17 & 21.5 days & Trace & 30 & (b) \\
\hline $\mathrm{Tc}(\mathrm{VII})$ & Solution 5 & Soil A & Batch & 0.54 & 0.65 & 21.5 days & Trace & 30 & (b) \\
\hline $\mathrm{Tc}(\mathrm{VII})$ & Solution 6 & Soil A & Batch & -0.51 & 1.2 & 21.5 days & Trace & 30 & (b) \\
\hline
\end{tabular}




\begin{tabular}{|c|c|c|c|c|c|c|c|c|c|}
\hline Species & $\begin{array}{l}\text { Aqueous } \\
\text { Phase }\end{array}$ & Solid Phase & Method & $\begin{array}{c}\mathrm{Kd} \\
(\mathrm{mL} / \mathrm{g})\end{array}$ & Std. Dev. & $\begin{array}{l}\text { Equil. } \\
\text { Time }\end{array}$ & $\begin{array}{l}\text { In. Soln. } \\
\text { Conc. }\end{array}$ & $\begin{array}{l}\text { Solution/ } \\
\text { Solid } \\
(\mathrm{mL} / \mathrm{g})\end{array}$ & Reference \\
\hline $\mathrm{Tc}(\mathrm{VII})$ & Solution 1 & Soil B & Batch & 0.54 & 1.61 & 8.5 days & Trace & 30 & (b) \\
\hline $\mathrm{Tc}(\mathrm{VII})$ & Solution 2 & Soil B & Batch & -0.02 & 0.99 & 8.5 days & Trace & 30 & (b) \\
\hline $\mathrm{Tc}(\mathrm{VII})$ & Solution 3 & Soil B & Batch & 0.85 & 0.32 & 8.5 days & Trace & 30 & (b) \\
\hline $\mathrm{Tc}(\mathrm{VII})$ & Solution 4 & Soil B & Batch & 1.27 & 1.67 & 8.5 days & Trace & 30 & (b) \\
\hline $\mathrm{Tc}(\mathrm{VII})$ & Solution 5 & Soil B & Batch & -0.96 & 0.37 & 8.5 days & Trace & 30 & (b) \\
\hline $\mathrm{Tc}(\mathrm{VII})$ & Solution 6 & Soil B & Batch & -0.09 & 0.76 & 8.5 days & Trace & 30 & (b) \\
\hline $\mathrm{Tc}(\mathrm{VII})$ & Solution 1 & Soil B & Batch & 0.07 & 0.32 & 21.5 days & Trace & 30 & (b) \\
\hline $\mathrm{Tc}(\mathrm{VII})$ & Solution 2 & Soil B & Batch & -1.62 & 0.6 & 21.5 days & Trace & 30 & (b) \\
\hline $\mathrm{Tc}(\mathrm{VII})$ & Solution 3 & Soil B & Batch & -0.31 & 0.3 & 21.5 days & Trace & 30 & (b) \\
\hline $\mathrm{Tc}(\mathrm{VII})$ & Solution 4 & Soil B & Batch & 0.06 & 0.68 & 21.5 days & Trace & 30 & (b) \\
\hline $\mathrm{Tc}(\mathrm{VII})$ & Solution 5 & Soil B & Batch & 0.52 & 0.51 & 21.5 days & Trace & 30 & (b) \\
\hline Tc(VII) & Solution 6 & Soil B & Batch & 0.38 & 1.23 & 21.5 days & Trace & 30 & (b) \\
\hline $\mathrm{Tc}(\mathrm{VII})$ & Solution L & Soil B & Batch & -3 & 2 & 7 days & Trace & 30 & (b) \\
\hline $\mathrm{Tc}(\mathrm{VII})$ & Solution H & Soil B & Batch & -1 & 0.3 & 7 days & Trace & 30 & (b) \\
\hline $\mathrm{Tc}(\mathrm{VII})$ & Solution L & Soil B & Column & 0.03 & 0.01 & 4 days & Trace & 30 & (b) \\
\hline $\mathrm{Tc}(\mathrm{VII})$ & Solution L & Soil A & Column & 0.04 & & 4 days & Trace & 30 & (b) \\
\hline $\mathrm{Tc}(\mathrm{VII})$ & Hanford GW2 & B850007A & Batch & -0.01 & 0.02 & 14 days & Trace & 2 & (c) \\
\hline $\mathrm{Tc}(\mathrm{VII})$ & Hanford GW2 & B850010A & Batch & -0.02 & 0.03 & 14 days & Trace & 2 & (c) \\
\hline $\mathrm{Tc}(\mathrm{VII})$ & Hanford GW2 & B850012A & Batch & 0.01 & 0 & 14 days & Trace & 2 & (c) \\
\hline $\mathrm{Tc}(\mathrm{VII})$ & Hanford GW2 & B850014A & Batch & -0.01 & 0.03 & 14 days & Trace & 2 & (c) \\
\hline $\mathrm{Tc}(\mathrm{VII})$ & Hanford GW2 & B850015A & Batch & 0 & 0.02 & 14 days & Trace & 2 & (c) \\
\hline $\mathrm{Tc}(\mathrm{VII})$ & Hanford GW2 & B850016A & Batch & -0.01 & 0.02 & 14 days & Trace & 2 & (c) \\
\hline $\mathrm{Tc}(\mathrm{VII})$ & Hanford GW2 & B850017A & Batch & -0.04 & 0.01 & 14 days & Trace & 2 & (c) \\
\hline $\mathrm{Tc}(\mathrm{VII})$ & Hanford GW2 & B850019A & Batch & 0.02 & 0.02 & 14 days & Trace & 2 & (c) \\
\hline $\mathrm{Tc}(\mathrm{VII})$ & Hanford GW2 & B850020A & Batch & 0 & 0.01 & 14 days & Trace & 2 & (c) \\
\hline $\mathrm{Tc}(\mathrm{VII})$ & Hanford GW2 & B850021A & Batch & 0 & 0.02 & 14 days & Trace & 2 & (c) \\
\hline $\mathrm{Tc}(\mathrm{VII})$ & Hanford GW2 & B850022A & Batch & 0 & 0.02 & 14 days & Trace & 2 & (c) \\
\hline $\mathrm{Tc}(\mathrm{VII})$ & Hanford GW2 & B850023A & Batch & -0.01 & 0.03 & 14 days & Trace & 2 & (c) \\
\hline $\mathrm{Tc}(\mathrm{VII})$ & Hanford GW2 & B850024A & Batch & 0 & 0.02 & 14 days & Trace & 2 & (c) \\
\hline Tc(VII) & Hanford GW2 & B850025A & Batch & 0.01 & 0.01 & 14 days & Trace & 2 & (c) \\
\hline $\mathrm{Tc}(\mathrm{VII})$ & Hanford GW2 & B850027A & Batch & 0 & 0.01 & 14 days & Trace & 2 & (c) \\
\hline $\mathrm{Tc}(\mathrm{VII})$ & Hanford GW2 & B850029A & Batch & -0.01 & 0.03 & 14 days & Trace & 2 & (c) \\
\hline $\mathrm{Tc}(\mathrm{VII})$ & Hanford GW2 & B850031A & Batch & -0.03 & 0.04 & 14 days & Trace & 2 & (c) \\
\hline $\mathrm{Tc}(\mathrm{VII})$ & Hanford GW2 & B850032A & Batch & -0.01 & 0.01 & 14 days & Trace & 2 & (c) \\
\hline
\end{tabular}




\begin{tabular}{|c|c|c|c|c|c|c|c|c|c|}
\hline Species & $\begin{array}{l}\text { Aqueous } \\
\text { Phase }\end{array}$ & Solid Phase & Method & $\begin{array}{c}\mathrm{Kd} \\
(\mathrm{mL} / \mathrm{g})\end{array}$ & Std. Dev. & $\begin{array}{c}\text { Equil. } \\
\text { Time }\end{array}$ & $\begin{array}{c}\text { In. Soln. } \\
\text { Conc. }\end{array}$ & $\begin{array}{l}\text { Solution/ } \\
\text { Solid } \\
(\mathrm{mL} / \mathrm{g})\end{array}$ & Reference \\
\hline $\mathrm{Tc}(\mathrm{VII})$ & Hanford GW2 & B850034A & Batch & -0.02 & 0.01 & 14 days & Trace & 2 & (c) \\
\hline $\mathrm{Tc}(\mathrm{VII})$ & Hanford GW2 & B850035A & Batch & -0.02 & 0.01 & 14 days & Trace & 2 & (c) \\
\hline $\mathrm{Tc}(\mathrm{VII})$ & GW-IS-0.05 & Trench AE-3 & Batch & -0.16 & 0.04 & 14 days & Trace & 2 & (d) \\
\hline $\mathrm{Tc}(\mathrm{VII})$ & GW-IS-0.10 & Trench AE-3 & Batch & -0.13 & 0 & 14 days & Trace & 2 & (d) \\
\hline $\mathrm{Tc}(\mathrm{VII})$ & GW-IS-0.50 & Trench AE-3 & Batch & -0.28 & 0.01 & 14 days & Trace & 2 & (d) \\
\hline $\mathrm{Tc}(\mathrm{VII})$ & GW-IS-1.00 & Trench AE-3 & Batch & 3.94 & 0.99 & 14 days & Trace & 2 & (d) \\
\hline $\mathrm{Tc}(\mathrm{VII})$ & GW-pH 8.1 & Trench AE-3 & Batch & -0.02 & 0.01 & 14 days & Trace & 2 & (d) \\
\hline $\mathrm{Tc}(\mathrm{VII})$ & GW-pH 9.9 & Trench AE-3 & Batch & 1.04 & 0.06 & 14 days & Trace & 2 & (d) \\
\hline $\mathrm{Tc}(\mathrm{VII})$ & GW-pH 10.2 & Trench AE-3 & Batch & 1.05 & 0.2 & 14 days & Trace & 2 & (d) \\
\hline $\mathrm{Tc}(\mathrm{VII})$ & GW-pH 11.0 & Trench AE-3 & Batch & 1.07 & 0.05 & 14 days & Trace & 2 & (d) \\
\hline $\mathrm{Tc}(\mathrm{VII})$ & GW-pH 11.9 & Trench AE-3 & Batch & 1.07 & 0.03 & 14 days & Trace & 2 & (d) \\
\hline $\mathrm{Tc}(\mathrm{VII})$ & HGW4 & Trench 94 & Batch & -0.03 & 0.006 & 7 & Trace & 2 & (e) \\
\hline $\mathrm{Tc}(\mathrm{VII})$ & HGW4 & Trench 94 & Batch & -0.044 & 0.013 & 266 & Trace & 2 & (e) \\
\hline $\mathrm{Tc}(\mathrm{VII})$ & HGW4 & Trench 94 & Batch & 0.057 & & 328 & Trace & 2 & (e) \\
\hline $\mathrm{Tc}(\mathrm{VII})$ & HGW4 & Trench 94 & Batch & 0.107 & 0.007 & 398 & Trace & 2 & (e) \\
\hline $\mathrm{Tc}(\mathrm{VII})$ & HGW4 & Trench AE-3 & Batch & -0.018 & 0.057 & 7 & Trace & 2 & (e) \\
\hline $\mathrm{Tc}(\mathrm{VII})$ & HGW4 & Trench AE-3 & Batch & -0.18 & 0.019 & 266 & Trace & 2 & (e) \\
\hline $\mathrm{Tc}(\mathrm{VII})$ & HGW4 & Trench AE-3 & Batch & 0.023 & 0.011 & 328 & Trace & 2 & (e) \\
\hline $\mathrm{Tc}(\mathrm{VII})$ & HGW4 & Trench AE-3 & Batch & 0.11 & 0.01 & 398 & Trace & 2 & (e) \\
\hline $\mathrm{Tc}(\mathrm{VII})$ & HGW4 & TSB-1 & Batch & -0.042 & 0.007 & 7 & Trace & 2 & (e) \\
\hline $\mathrm{Tc}(\mathrm{VII})$ & HGW4 & TSB-1 & Batch & -0.182 & 0.015 & 266 & Trace & 2 & (e) \\
\hline $\mathrm{Tc}(\mathrm{VII})$ & HGW4 & TSB-1 & Batch & 0.049 & 0.029 & 328 & Trace & 2 & (e) \\
\hline $\mathrm{Tc}(\mathrm{VII})$ & HGW4 & TSB-1 & Batch & 0.052 & 0.031 & 398 & Trace & 2 & (e) \\
\hline \multicolumn{10}{|c|}{\begin{tabular}{|l} 
(a) Serne et al. 1993. \\
(b) Gee and Campbell 1980. \\
(c) Kaplan et al. 1998a. \\
(d) Kaplan et al. $1998 \mathrm{~b}$. \\
(e) Kaplan et al. 1996.
\end{tabular}} \\
\hline
\end{tabular}


Table 16. Contaminant Distribution Coefficient Data for Uranium

\begin{tabular}{|c|c|c|c|c|c|c|c|c|c|}
\hline Species & Aqueous Phase & Solid Phase & Method & $\begin{array}{c}\mathrm{Kd} \\
(\mathrm{mL} / \mathrm{g})\end{array}$ & Std. Dev. & $\begin{array}{c}\text { Equil. } \\
\text { Time }\end{array}$ & $\begin{array}{c}\text { In. Soln. } \\
\text { Conc. }\end{array}$ & $\begin{array}{c}\text { Solution/ } \\
\text { Solid } \\
(\mathrm{mL} / \mathrm{g})\end{array}$ & Reference \\
\hline U(VI) & Hanford GW & CGS-1 & Batch & 1.6 & 3.4 & 4 days & 2.10E-07 & 30 & (a) \\
\hline $\mathrm{U}(\mathrm{VI})$ & Hanford GW & CGS-1 & Batch & -1.5 & 4.5 & 14 days & $2.10 \mathrm{E}-07$ & 30 & (a) \\
\hline $\mathrm{U}(\mathrm{VI})$ & Hanford GW & CGS-1 & Batch & 5.2 & 1.8 & 35 days & $2.10 \mathrm{E}-07$ & 30 & (a) \\
\hline $\mathrm{U}(\mathrm{VI})$ & $\begin{array}{l}\text { Acid Proc } \\
\text { Waste }\end{array}$ & CGS-1 & Batch & 147 & 39 & 4 days & $2.10 \mathrm{E}-07$ & 30 & (a) \\
\hline $\mathrm{U}(\mathrm{VI})$ & $\begin{array}{l}\text { Acid Proc } \\
\text { Waste }\end{array}$ & CGS-1 & Batch & 486 & 25 & 14 days & 2.10E-07 & 30 & (a) \\
\hline $\mathrm{U}(\mathrm{VI})$ & $\begin{array}{l}\text { Acid Proc } \\
\text { Waste }\end{array}$ & CGS-1 & Batch & 1000 & 53 & 35 days & 2.10E-07 & 30 & (a) \\
\hline $\mathrm{U}(\mathrm{VI})$ & Hanford GW & TBS-1 & Batch & 104 & 28 & 4 days & $2.10 \mathrm{E}-07$ & 30 & (a) \\
\hline $\mathrm{U}(\mathrm{VI})$ & Hanford GW & TBS-1 & Batch & 74 & 18 & 14 days & $2.10 \mathrm{E}-07$ & 30 & (a) \\
\hline $\mathrm{U}(\mathrm{VI})$ & Hanford GW & TBS-1 & Batch & 60.5 & 6.1 & 35 days & 2.10E-07 & 30 & (a) \\
\hline $\mathrm{U}(\mathrm{VI})$ & $\begin{array}{l}\text { Acid Proc } \\
\text { Waste }\end{array}$ & TBS-1 & Batch & 109 & 66 & 4 days & $2.10 \mathrm{E}-07$ & 30 & (a) \\
\hline $\mathrm{U}(\mathrm{VI})$ & $\begin{array}{l}\text { Acid Proc } \\
\text { Waste }\end{array}$ & TBS-1 & Batch & 223 & 57 & 14 days & 2.10E-07 & 30 & (a) \\
\hline $\mathrm{U}(\mathrm{VI})$ & $\begin{array}{l}\text { Acid Proc } \\
\text { Waste }\end{array}$ & TBS-1 & Batch & 579 & 330 & 35 days & 2.10E-07 & 30 & (a) \\
\hline $\mathrm{U}(\mathrm{VI})$ & Hanford GW & Trench-8 & Batch & 1.9 & 1.4 & 5 days & $2.10 \mathrm{E}-07$ & 30 & (a) \\
\hline $\mathrm{U}(\mathrm{VI})$ & Hanford GW & Trench-8 & Batch & 2.4 & 0.6 & 44 days & 2.10E-07 & 30 & (a) \\
\hline $\mathrm{U}(\mathrm{VI})$ & Hanford GW2 & B850007A & Batch & 0.94 & 0.12 & 14 days & 8.40E-07 & 2 & (b) \\
\hline $\mathrm{U}(\mathrm{VI})$ & Hanford GW2 & B850010A & Batch & 0.67 & 0.08 & 14 days & 8.40E-07 & 2 & (b) \\
\hline $\mathrm{U}(\mathrm{VI})$ & Hanford GW2 & B850012A & Batch & 0.64 & 0.1 & 14 days & $8.40 \mathrm{E}-07$ & 2 & (b) \\
\hline U(VI) & Hanford GW2 & B850014A & Batch & 0.67 & 0.05 & 14 days & $8.40 \mathrm{E}-07$ & 2 & (b) \\
\hline $\mathrm{U}(\mathrm{VI})$ & Hanford GW2 & B850015A & Batch & 0.74 & 0.05 & 14 days & 8.40E-07 & 2 & (b) \\
\hline $\mathrm{U}(\mathrm{VI})$ & Hanford GW2 & B850016A & Batch & 0.57 & 0.04 & 14 days & $8.40 \mathrm{E}-07$ & 2 & (b) \\
\hline $\mathrm{U}(\mathrm{VI})$ & Hanford GW2 & B850017A & Batch & 0.68 & 0.15 & 14 days & 8.40E-07 & 2 & (b) \\
\hline $\mathrm{U}(\mathrm{VI})$ & Hanford GW2 & B850019A & Batch & 0.51 & 0.08 & 14 days & $8.40 \mathrm{E}-07$ & 2 & (b) \\
\hline $\mathrm{U}(\mathrm{VI})$ & Hanford GW2 & B850020A & Batch & 0.65 & 0.08 & 14 days & 8.40E-07 & 2 & (b) \\
\hline $\mathrm{U}(\mathrm{VI})$ & Hanford GW2 & B850021A & Batch & 0.57 & 0.09 & 14 days & 8.40E-07 & 2 & (b) \\
\hline $\mathrm{U}(\mathrm{VI})$ & Hanford GW2 & B850022A & Batch & 0.59 & 0.08 & 14 days & 8.40E-07 & 2 & (b) \\
\hline $\mathrm{U}(\mathrm{VI})$ & Hanford GW2 & B850023A & Batch & 0.58 & 0.05 & 14 days & 8.40E-07 & 2 & (b) \\
\hline $\mathrm{U}(\mathrm{VI})$ & Hanford GW2 & B850024A & Batch & 0.55 & 0.05 & 14 days & $8.40 \mathrm{E}-07$ & 2 & (b) \\
\hline $\mathrm{U}(\mathrm{VI})$ & Hanford GW2 & B850025A & Batch & 0.56 & 0.07 & 14 days & 8.40E-07 & 2 & (b) \\
\hline $\mathrm{U}(\mathrm{VI})$ & Hanford GW2 & B850027A & Batch & 0.59 & 0.08 & 14 days & 8.40E-07 & 2 & (b) \\
\hline U(VI) & Hanford GW2 & B850029A & Batch & 0.5 & 0.13 & 14 days & 8.40E-07 & 2 & (b) \\
\hline
\end{tabular}




\begin{tabular}{|c|c|c|c|c|c|c|c|c|c|}
\hline Species & Aqueous Phase & Solid Phase & Method & $\begin{array}{c}\mathrm{Kd} \\
(\mathrm{mL} / \mathrm{g})\end{array}$ & Std. Dev. & $\begin{array}{l}\text { Equil. } \\
\text { Time }\end{array}$ & $\begin{array}{l}\text { In. Soln. } \\
\text { Conc. }\end{array}$ & $\begin{array}{c}\text { Solution/ } \\
\text { Solid } \\
(\mathrm{mL} / \mathrm{g})\end{array}$ & Reference \\
\hline $\mathrm{U}(\mathrm{VI})$ & Hanford GW2 & B850031A & Batch & 0.3 & 0.12 & 14 days & $8.40 \mathrm{E}-07$ & 2 & (b) \\
\hline $\mathrm{U}(\mathrm{VI})$ & Hanford GW2 & B850032A & Batch & 0.7 & 0.11 & 14 days & $8.40 \mathrm{E}-07$ & 2 & (b) \\
\hline $\mathrm{U}(\mathrm{VI})$ & Hanford GW2 & B850034A & Batch & 0.61 & 0.16 & 14 days & $8.40 \mathrm{E}-07$ & 2 & (b) \\
\hline $\mathrm{U}(\mathrm{VI})$ & Hanford GW2 & B850035A & Batch & 0.68 & 0.13 & 14 days & $8.40 \mathrm{E}-07$ & 2 & (b) \\
\hline $\mathrm{U}(\mathrm{VI})$ & Hanford GW & Trench-8 & $\begin{array}{l}\text { Col } \\
100 \% \text { Sat }\end{array}$ & 2 & & $12.4 \mathrm{hr}$ & $1.40 \mathrm{E}-07$ & & (c) \\
\hline $\mathrm{U}(\mathrm{VI})$ & Hanford GW & Trench-8 & $\begin{array}{l}\text { Col } \\
100 \% \text { Sat }\end{array}$ & 0.5 & & $0.6 \mathrm{hr}$ & $1.40 \mathrm{E}-07$ & & (c) \\
\hline $\mathrm{U}(\mathrm{VI})$ & Hanford GW & Trench-8 & \begin{tabular}{|l|} 
Col \\
$100 \%$ Sat \\
\end{tabular} & 2.7 & & $5.9 \mathrm{hr}$ & $1.40 \mathrm{E}-07$ & & (c) \\
\hline $\mathrm{U}(\mathrm{VI})$ & Hanford GW & Trench-8 & Col 65\% Sat & 1 & & $32.4 \mathrm{hr}$ & $1.40 \mathrm{E}-07$ & & (c) \\
\hline $\mathrm{U}(\mathrm{VI})$ & Hanford GW & Trench-8 & $\begin{array}{l}\text { Col 70\%Sat } \\
\text { UFA }\end{array}$ & 0.5 & & $0.5 \mathrm{hr}$ & $2.60 \mathrm{E}-06$ & & (c) \\
\hline $\mathrm{U}(\mathrm{VI})$ & Hanford GW & Trench- 8 & $\begin{array}{l}\text { Col 24\%Sat } \\
\text { UFA }\end{array}$ & 0.2 & & $0.6 \mathrm{hr}$ & $2.60 \mathrm{E}-06$ & & (c) \\
\hline $\mathrm{U}(\mathrm{VI})$ & Hanford GW & Trench-8 & Col 63\%Sat & 1.1 & & $77 \mathrm{hr}$ & $1.40 \mathrm{E}-07$ & & (c) \\
\hline $\mathrm{U}(\mathrm{VI})$ & Hanford GW & Trench-8 & Col $43 \%$ Sat & 1.1 & & $31.5 \mathrm{hr}$ & $1.40 \mathrm{E}-07$ & & (c) \\
\hline $\mathrm{U}(\mathrm{VI})$ & Hanford GW & Trench-8 & $\begin{array}{l}\text { Col 29\%Sat } \\
\text { UFA }\end{array}$ & 0.6 & & $20.6 \mathrm{hr}$ & $2.60 \mathrm{E}-06$ & & (c) \\
\hline $\mathrm{U}(\mathrm{VI})$ & Hanford GW & Trench-8 & $\begin{array}{l}\text { Col } 29 \% \text { Sat } \\
\text { UFA }\end{array}$ & 0.6 & & $20.6 \mathrm{hr}$ & $2.60 \mathrm{E}-06$ & & (c) \\
\hline $\mathrm{U}(\mathrm{VI})$ & Hanford GW2 & MCS & $\begin{array}{l}\text { Col 30\%Sat } \\
\text { UFA } \\
\end{array}$ & 0.48 & & $0.13 \mathrm{hr}$ & $1.70 \mathrm{E}-06$ & & (d) \\
\hline $\mathrm{U}(\mathrm{VI})$ & Hanford GW2 & MCS & $\begin{array}{l}\text { Col } 12 \% \text { Sat } \\
\text { UFA }\end{array}$ & 0.16 & & $0.088 \mathrm{hr}$ & $1.70 \mathrm{E}-06$ & & (d) \\
\hline $\mathrm{U}(\mathrm{VI})$ & Hanford GW2 & TBS & $\begin{array}{l}\text { Col } 66 \% \text { Sat } \\
\text { UFA }\end{array}$ & 1.42 & & $0.32 \mathrm{hr}$ & $1.70 \mathrm{E}-06$ & & (d) \\
\hline $\mathrm{U}(\mathrm{VI})$ & Hanford GW2 & TBS & $\begin{array}{l}\text { Col } 22 \% \text { Sat } \\
\text { UFA }\end{array}$ & 0.39 & & $0.17 \mathrm{hr}$ & $1.70 \mathrm{E}-06$ & & (d) \\
\hline $\mathrm{U}(\mathrm{VI})$ & Hanford GW2 & WSL & $\begin{array}{l}\text { Col } 83 \% \text { Sat } \\
\text { UFA }\end{array}$ & 4.05 & & $0.38 \mathrm{hr}$ & $1.70 \mathrm{E}-06$ & & (d) \\
\hline $\mathrm{U}(\mathrm{VI})$ & Hanford GW2 & WSL & $\begin{array}{l}\text { Col } 41 \% \text { Sat } \\
\text { UFA }\end{array}$ & 1.81 & & $0.34 \mathrm{hr}$ & $1.70 \mathrm{E}-06$ & & (d) \\
\hline $\mathrm{U}(\mathrm{VI})$ & Hanford GW2 & TBS & $\begin{array}{l}\text { Col } \\
100 \% \text { Sat } \\
\text { UFA } \\
\end{array}$ & 0.79 & & $0.24 \mathrm{hr}$ & $1.70 \mathrm{E}-06$ & & (d) \\
\hline $\mathrm{U}(\mathrm{VI})$ & Hanford GW2 & WSL & $\begin{array}{l}\text { Col } \\
100 \% \text { Sat } \\
\text { UFA }\end{array}$ & 1.83 & & $0.23 \mathrm{hr}$ & $1.70 \mathrm{E}-06$ & & (d) \\
\hline $\mathrm{U}(\mathrm{VI})$ & $\begin{array}{c}0.0 \mathrm{mM} \\
\mathrm{NaClO}_{4} \\
\end{array}$ & Trench AE-3 & Batch & 1.99 & 0.1 & 14 days & $7.10 \mathrm{E}-07$ & 2 & (e) \\
\hline $\mathrm{U}(\mathrm{VI})$ & $\begin{array}{l}0.3 \mathrm{mM} \\
\mathrm{NaClO}_{4}\end{array}$ & Trench AE-3 & Batch & 1.92 & 0.13 & 14 days & 7.10E-07 & 2 & (e) \\
\hline
\end{tabular}




\begin{tabular}{|c|c|c|c|c|c|c|c|c|c|}
\hline Species & Aqueous Phase & Solid Phase & Method & $\begin{array}{c}\mathrm{Kd} \\
(\mathrm{mL} / \mathrm{g})\end{array}$ & Std. Dev. & $\begin{array}{c}\text { Equil. } \\
\text { Time }\end{array}$ & $\begin{array}{c}\text { In. Soln. } \\
\text { Conc. }\end{array}$ & $\begin{array}{c}\text { Solution/ } \\
\text { Solid } \\
(\mathrm{mL} / \mathrm{g})\end{array}$ & Reference \\
\hline U(VI) & $\begin{array}{l}1.0 \mathrm{mM} \\
\mathrm{NaClO}_{4}\end{array}$ & Trench AE-3 & Batch & 1.91 & 0.17 & 14 days & $7.10 \mathrm{E}-07$ & 2 & (e) \\
\hline $\mathrm{U}(\mathrm{VI})$ & $\begin{array}{l}1.7 \mathrm{mM} \\
\mathrm{NaClO}_{4}\end{array}$ & Trench AE-3 & Batch & 2.1 & 0.22 & 14 days & $7.10 \mathrm{E}-07$ & 2 & (e) \\
\hline U(VI) & $\begin{array}{l}4.1 \mathrm{mM} \\
\mathrm{NaClO}_{4}\end{array}$ & Trench AE-3 & Batch & 2.25 & 0.15 & 14 days & 7.10E-07 & 2 & (e) \\
\hline U(VI) & $\begin{array}{l}14.0 \mathrm{mM} \\
\mathrm{NaClO}_{4}\end{array}$ & Trench AE-3 & Batch & 2.44 & 0.44 & 14 days & 7.10E-07 & 2 & (e) \\
\hline $\mathrm{U}(\mathrm{VI})$ & HGW pH 8.0 & Trench AE-3 & Batch & 1.26 & 0.12 & 14 days & $7.10 \mathrm{E}-07$ & 2 & (e) \\
\hline $\mathrm{U}(\mathrm{VI})$ & HGW pH 8.0 & Trench AE-3 & Batch & 1.27 & 0.55 & 14 days & $7.10 \mathrm{E}-07$ & 2 & (e) \\
\hline $\mathrm{U}(\mathrm{VI})$ & HGW pH 8.0 & Trench AE-3 & Batch & 2.86 & 0.53 & 14 days & $7.10 \mathrm{E}-07$ & 2 & (e) \\
\hline $\mathrm{U}(\mathrm{VI})$ & HGW pH 8.0 & Trench AE-3 & Batch & 3.09 & 0.25 & 14 days & $7.10 \mathrm{E}-07$ & 2 & (e) \\
\hline U(VI) & HGW pH 8.2 & Trench AE-3 & Batch & 3.51 & 0.26 & 14 days & $7.10 \mathrm{E}-07$ & 2 & (e) \\
\hline U(VI) & HGW pH 11.0 & Trench AE-3 & Batch & $\begin{array}{c}546 \\
\text { (prec) }\end{array}$ & 70 & 14 days & 7.10E-07 & 2 & (e) \\
\hline U(VI) & HGW4 & Trench 94 & Batch & 0.45 & 0.04 & 7 & $1.30 \mathrm{E}-06$ & 2 & (e) \\
\hline $\mathrm{U}(\mathrm{VI})$ & HGW4 & Trench 94 & Batch & 0.79 & 0.01 & 266 & $1.30 \mathrm{E}-06$ & 2 & (e) \\
\hline $\mathrm{U}(\mathrm{VI})$ & HGW4 & Trench 94 & Batch & 0.84 & 0.14 & 328 & $1.30 \mathrm{E}-06$ & 2 & (e) \\
\hline U(VI) & HGW4 & Trench 94 & Batch & 1.12 & 0.02 & 398 & $1.30 \mathrm{E}-06$ & 2 & (e) \\
\hline $\mathrm{U}(\mathrm{VI})$ & HGW4 & Trench 8B & Batch & 1.62 & 0.12 & 7 & $1.30 \mathrm{E}-06$ & 2 & (e) \\
\hline U(VI) & HGW4 & Trench 8B & Batch & 3.24 & 0.4 & 266 & $1.30 \mathrm{E}-06$ & 2 & (e) \\
\hline $\mathrm{U}(\mathrm{VI})$ & HGW4 & Trench 8B & Batch & 3.41 & 0.25 & 328 & $1.30 \mathrm{E}-06$ & 2 & (e) \\
\hline U(VI) & HGW4 & Trench 8B & Batch & 3.49 & 0.23 & 398 & $1.30 \mathrm{E}-06$ & 2 & (e) \\
\hline $\mathrm{U}(\mathrm{VI})$ & HGW4 & TSB & Batch & 1.23 & 0.1 & 7 & $1.30 \mathrm{E}-06$ & 2 & (e) \\
\hline $\mathrm{U}(\mathrm{VI})$ & HGW4 & TSB & Batch & 1.6 & 1.1 & 266 & $1.30 \mathrm{E}-06$ & 2 & (e) \\
\hline U(VI) & HGW4 & TSB & Batch & 2.11 & 0.17 & 328 & 1.30E-06 & 2 & (e) \\
\hline $\mathrm{U}(\mathrm{VI})$ & HGW4 & TSB & Batch & 2.1 & 0.01 & 398 & $1.30 \mathrm{E}-06$ & 2 & (e) \\
\hline \multicolumn{10}{|c|}{$\begin{array}{l}\text { (a) Serne et al. 1993. } \\
\text { (b) Kaplan et al. 1998a. } \\
\text { (c) Lindenmeier et al. } 1995 . \\
\text { (d) Gamerdinger et al. } 1998 . \\
\text { (e) Kaplan et al. 1996. }\end{array}$} \\
\hline
\end{tabular}


Table 17. Contaminant Distribution Coefficient Data for Carbon Tetrachloride

\begin{tabular}{|c|c|c|c|c|c|c|c|c|c|}
\hline Species & Aqueous Phase & Solid Phase & Method & $\begin{array}{c}\mathrm{Kd} \\
(\mathrm{mL} / \mathrm{g})\end{array}$ & Std. Dev. & $\begin{array}{l}\text { Equil. } \\
\text { Time }\end{array}$ & $\begin{array}{l}\text { In. Soln. } \\
\text { Conc. }\end{array}$ & $\begin{array}{l}\text { Solution/Solid } \\
(\mathrm{mL} / \mathrm{g})\end{array}$ & Reference \\
\hline $\mathrm{CCl}_{4}$ & Hanford GW & 299-W11-27 (200ft) & Batch & -1.8 & & 4 days & $10000 \mathrm{ppb}$ & 40 & (a) \\
\hline $\mathrm{CCl}_{4}$ & Hanford GW & 299-W11-27 (200 ft) & Batch & 0.0 & & 4 days & $10000 \mathrm{ppb}$ & 40 & (a) \\
\hline $\mathrm{CCl}_{4}$ & Hanford GW & 299-W11-27 (200 ft) & Batch & -0.2 & & 4 days & $5000 \mathrm{ppb}$ & 40 & (a) \\
\hline $\mathrm{CCl}_{4}$ & Hanford GW & 299-W11-27 (200 ft) & Batch & 0.3 & & 4 days & $5000 \mathrm{ppb}$ & 40 & (a) \\
\hline $\mathrm{CCl}_{4}$ & Hanford GW & 299-W11-27 (200 ft) & Batch & -1.5 & & 4 days & $500 \mathrm{ppb}$ & 40 & (a) \\
\hline $\mathrm{CCl}_{4}$ & Hanford GW & 299-W11-27 (200 ft) & Batch & -1.5 & & 4 days & $500 \mathrm{ppb}$ & 40 & (a) \\
\hline $\mathrm{CCl}_{4}$ & Hanford GW & 299-W11-27 (200 ft) & Batch & -1.6 & & 4 days & $50 \mathrm{ppb}$ & 40 & (a) \\
\hline $\mathrm{CCl}_{4}$ & Hanford GW & 299-W11-27 (200 ft) & Batch & -0.6 & & 4 days & $50 \mathrm{ppb}$ & 40 & (a) \\
\hline $\mathrm{CCl}_{4}$ & Hanford GW & 299-W11-27 (200 ft) & Batch & -0.5 & & 4 days & $5 \mathrm{ppb}$ & 40 & (a) \\
\hline $\mathrm{CCl}_{4}$ & Hanford GW & 299-W11-27 (200 ft) & Batch & -0.6 & & 4 days & $5 \mathrm{ppb}$ & 40 & (a) \\
\hline
\end{tabular}




\subsection{References}

Ames, L. L. and D. Rai. 1978. Radionuclide Interactions with Rock and Soil Media. Volume I. PB292460, U.S. Environmental Protection Agency, Office of Radiation Programs, Las Vegas, Nevada.

Brusseau, M. L., T. Larsen, and T. H. Christensen. 1991. "Rate-Limited Sorption and Nonequilibrium Transport of Organic Chemicals in Low Organic Carbon Aquifer Materials." Water Resour. Res. 27(6):1137-1145.

Cantrell, K. J. and R. J. Serne. 1993. Adsorption of ${ }^{60} \mathrm{Co},{ }^{90} \mathrm{Sr},{ }^{99} \mathrm{Tc},{ }^{137} \mathrm{Cs}$, Pu, and Cyanide on 200-BP-1 Sediment. PNL-8612, Pacific Northwest Laboratory, Richland, Washington.

Chin, Y., C. S. Peven, and W. J. Weber, Jr. 1988. "Estimating Soil/Sediment Partition Coefficients for Organic Compounds by High Performance Reverse Phase Liquid Chromatography." Wat. Res. 22(7):871-881.

Curtis, G. P., R. V. Roberts, and M. Reinhard. 1986. "A Natural Gradient Experiment on Solute Transport in a Sand Aquifer 4. Sorption of Organic Solutes and its Influence on Mobility." Water Resour. Res 22(13):2059-2067.

Davis, J. A. 2001. Surface Complexation Modeling of Uranium(VI) Adsorption on Natural Mineral Assemblages. NUREG/CR-6708, U.S. Nuclear Regulatory Commission, Washington, D.C.

DeLamare, M. A. 2000. Groundwater/Vadose Zone Integration Project Systems Engineering Management Plan. BHI(I)01347, Rev. 0, Bechtel Hanford, Inc., Richland, Washington.

Delegard, C. H. and G. S. Barney. 1983. Effects of Hanford High-Level Waste Components on Sorption of Cobalt, Strontium, Neptunium, Plutonium, and Americium of Hanford Sediments. RHO-RE-ST-1 P, Rockwell Hanford Operations, Richland, Washington.

DOE (U.S. Department of Energy). 1999. Groundwater/Vadose Zone Integration Project Background Information and State of Knowledge. DOE/RL-98-48, Richland Operations Office, Richland, Washington.

DOE (U.S. Department of Energy). 2000. Groundwater/Vadose Zone Integration Project Science and Technology Summary Description. DOE-RL-98-48 Vol. III, Rev. 1, Richland Operations Office, Richland, Washington.

Freeman, E. J., R. Khaleel, and P. R. Heller. 2001. A Catalog of Vadose Zone Hydraulic Properties for the Hanford Site. PNNL-13672, Pacific Northwest National Laboratory, Richland, Washington.

Gee, G. W. and A. C. Campbell. 1980. Monitoring and Physical Characterization of Unsaturated Zone Transport - Laboratory Analysis. PNL-3304, Pacific Northwest Laboratory, Richland, Washington. 
Hajek, B. F. and L. L. Ames. 1966. Trace Strontium and Cesium Distribution Coefficients: Batch and Column Determination. BNWL-SA-843, Battelle Pacific Northwest Laboratories, Richland, Washington.

Hajek, B. F. and K. C. Knoll. 1966. Disposal Characteristics of Plutonium and Americium in a High Salt Acid Waste. BNWL-CC-649, Battelle Pacific Northwest Laboratories, Richland, Washington.

Gamerdinger, A. P., D. I. Kaplan, and C. T. Resch. 1998. Uranium (VI) Sorption and Transport in Unsaturated, Subsurface Hanford Site Sediments - Effect of Moisture Content and Sediment Texture. PNNL-11975, Pacific Northwest National Laboratory, Richland, Washington.

Horton, D. G., G. V. Last, T. J Gilmore, and B. N. Bjornstad. 2001. A Catalog of Geologic Data for the Hanford Site. PNNL-13653, Pacific Northwest National Laboratory, Richland, Washington.

Kaplan, D. I, K. E. Parker, and I. V. Kutynakov. 1998a. Radionuclide Distribution Coefficients for Sediments Collected from Borehole 299-E17-21: Final Report for Subtask 1a. PNNL-11966, Pacific Northwest National Laboratory, Richland, Washington.

Kaplan, D. I., K. E. Parker, and R. D. Orr. 1998b. Effects of High-pH and High-Ionic-Strength Groundwater on Iodide, Pertechnetate, and Selenate Sorption to Hanford Sediments: Final Report for Subtask 3a. PNNL-11964, Pacific Northwest National Laboratory, Richland, Washington.

Kaplan, D. I. and R. J. Serne. 2000. Geochemical Data Package for the Hanford Immobilized LowActivity Tank Waste Performance Assessment (ILAW PA). PNNL-13037, Rev. 1, Pacific Northwest National Laboratory, Richland, Washington.

Kaplan, D. I., R. J. Serne, A. T. Owen, J. A. Conca, T. W. Wietsma, and T. L. Gervais. 1996. Radionuclide Adsorption Distribution Coefficients Measured in Hanford Sediments for the Low-Level Waste Performance Assessment Project. PNNL-11485, Pacific Northwest National Laboratory, Richland, Washington.

Kaplan, D. I., I. V. Kutnyakov, A. P. Gamerdinger, R. J. Serne, and K. E. Parker. 2000. "GravelCorrected Kd Values." Ground Water. 38(6):851-857.

Langmuir, D. 1997. Aqueous Environmental Geochemistry. Prentice Hall, Upper Saddle River, New Jersey.

Last, G. V. and D. G. Horton. 2000. Review of Geophysical Characterization Methods Used at the Hanford Site. PNNL-13149, Pacific Northwest National Laboratory, Richland, Washington.

Lindenmeier, C. W., R. J. Serne, J. L. Conca, A. T. Owen, and M. I. Wood. 1995. Solid Waste Leach Characteristics and Contaminant-Sediment Interactions Volume 2: Contaminant Transport Under Unsaturated Moisture Contents. PNL-10722, Pacific Northwest Laboratory, Richland, Washington. Lindsay, W. L. 1979. Chemical Equilibria in Soils. John Wiley and Sons, New York. 
Mackay, D. M., D. L. Freyberg, and R. V. Roberts. 1986. “A Natural Gradient Experiment on Solute Transport in a Sand Aquifer 1. Approach and Overview of Plume Movement." Water Resour. Res 22(13):2017-2029.

Payne, T. E. and T. D. Waite. 1991. "Surface Complexation Modeling of Uranium Sorption Data Obtained by Isotope Exchange Techniques.” Radiochim. Acta. 52/53:487-493.

Relyea, J. F., R. J. Serne, and D. Rai. 1980. Methods for Determining Radionuclide Retardation Factors: Status Report. PNL-3349, Pacific Northwest Laboratory, Richland, Washington.

Rhoades, K., B. N. Bjornstad, R. E. Lewis, S. S. Teel, K. J. Cantrell, R. J. Serne, L. H. Sawyer, J. L. Smoot, J. E. Smoot, J. E. Szecsody, M. S. Wigmosta, and S. K. Wurstner. 1992. Estimation of the Release and Migration of Lead Through Soils and Groundwater at the Hanford Site 218-E-12B Burial Ground. PNL-8356, Pacific Northwest Laboratory, Richland, Washington.

Rhoades, K., B. N. Bjornstad, R. E. Lewis, S. S. Teel, K. J. Cantrell, R. J. Serne, L. H. Sawyer, J. L. Smoot, J. E. Smoot, J. E. Szecsody, M. S. Wigmosta, and S. K. Wurstner. 1994. Estimation of the Release and Migration of Nickel Through Soils and Groundwater at the Hanford Site 218-E-12B Burial Ground. PNL-9791, Pacific Northwest Laboratory, Richland, Washington.

Rhodes, D. W. 1952. Preliminary Studies of Plutonium Adsorption in Hanford Soil. HW-24548, Hanford Works, Richland, Washington.

Rhodes, D. W. 1957. “The Adsorption of Pu by Soil.” Soil Sci. 84:465-471.

Riley, R. G. and C. A. Lo Presti. 2000. Data Catalog for Models Simulating Release of Contaminants from Hanford Site Waste Sources. PNNL-13666, Pacific Northwest National Laboratory, Richland, Washington.

Roberts, P. V., M. N. Goltz, and D. M. Mackay. 1986. “A Natural Gradient Experiment on Solute Transport in a Sand Aquifer 3. Retardation Estimates and Mass Balances for Organic Solutes." Water Resour. Res 22(13):2047-2058.

Routson, R. C. 1973. A Review of Studies on Soil-Waste Relationships on the Hanford Reservation from 1944 to 1967. BNWL-1464, Battelle Pacific Northwest Laboratories, Richland, Washington.

Routson, R. C., G. Jansen, and A. V. Robinson. 1975. Sorption of 99Tc, 237Np and 241 Am on Two Subsoils from Differing Weathering Intensity Areas. BNWL-1889, Battelle Pacific Northwest Laboratories, Richland, Washington.

Routson, R. C., G. Jansen, and A. V. Robinson. 1976. "241-Am, 237-Np, and 99-Tc Sorption on Two United States Subsoils from Differing Weathering Intensity Areas." Health Phys. 33:311-317. 
Serne, R. J. and V. L. LeGore. 1996. Strontium-90 Adsorption-Desorption Properties and Sediment Characterization at the $100 \mathrm{~N}$-Area. PNL-10899, Pacific Northwest Laboratory, Richland, Washington.

Serne, R. J., J. L. Conca, V. L. LeGore, K. J. Cantrell, C. W. Lindenmeier, J. A. Campbell, J. E. Amonette, and M. I. Wood. 1993. Solid-Waste Leach Characteristics and Contaminant-Sediment Interactions. Volume 1: Batch Leach and Adsorption Tests and Sediment Characterization. PNL-8889, Vol 1, Pacific Northwest Laboratory, Richland, Washington.

Serne, R. J., J. M. Zachara, and D. S. Burke. 1998. Chemical Information on Tank Supernatants, Cs Adsorption from Tank Liquids onto Hanford Sediments, and Field Observations of Cs Migration from Past Tank Leaks. PNNL-11495, Pacific Northwest National Laboratory, Richland, Washington.

Sheppard, J. C., J. A. Kittrick, and T. L. Hart. 1976. Determination of Distribution Ratios and Diffusion Coefficients of Neptunium, Americium, and Curium in Soil-Aquatic Environments. RLO-2221-T-12-2 (WSU 76/13-33), Washington State University, Pullman, Washington.

Soler, L., G. V. Last, B. A. Napier, V. J. Rohay, and F. J. Schelling. 2001. The Application of Feature, Event and Process Methodology at the Hanford Site. BHI(I)01573, Bechtel Hanford, Inc. Richland, Washington.

Stumm, W. and J. J. Morgan. 1996. Aquatic Chemistry. Chemical Equilibria and Rates in Natural Waters. John Wiley and Sons, New York.

Truex, M. J., C. J. Murray, C. R. Cole, R. J. Cameron, M. D. Johnson, R. S. Sheen, and C. D. Johnson. 2001. Assessment of Carbon Tetrachloride Groundwater Transport in Support of the Hanford Carbon Tetrachloride Innovative Technology Demonstration Program. PNNL-13560, Pacific Northwest National Laboratory, Richland, Washington.

U.S. Environmental Protection Agency (EPA). 1999. Understanding Variation in Partition Coefficient, $K_{d}$,Values: Volume I. The $K_{d}$ Model, Methods of Measurement, and Application of Chemical Reaction Codes. EPA 402-R-99-004A, prepared for the U.S. Environmental Protection Agency, Washington, D.C., by K. M. Krupka, D. I. Kaplan, G. Whelan, R. J. Serne, and S. V. Mattigod, Pacific Northwest National Laboratory, Richland, Washington.

Zachara, J. M., S. C. Smith, C. Liu, J. P. McKinley, R. J. Serne, and P. L. Gassman. 2002. "Sorption of $\mathrm{Cs}^{+}$to Micaceous Subsurface Sediments from the Hanford Site, USA." Geochim. Cosmochim. Acta, 66:193-211. 


\section{Appendix A}

\section{Aqueous Phase (Solution) Characteristics}


Table A.1. Solution Characteristics






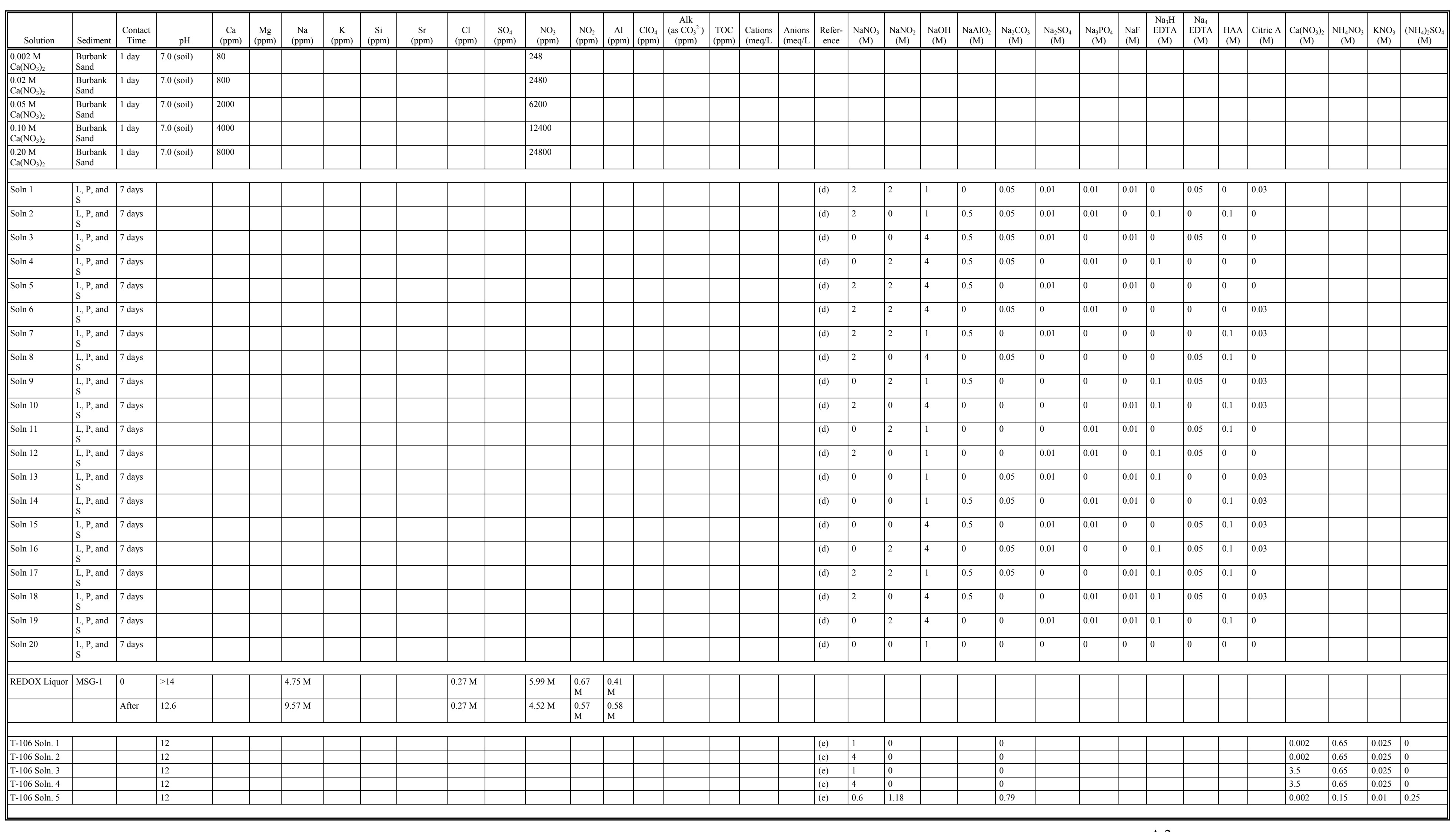




\begin{tabular}{|c|c|c|c|c|c|c|c|c|c|c|c|c|c|c|c|c|c|c|c|c|c|c|c|c|c|c|c|c|c|c|c|c|c|c|c|}
\hline \begin{tabular}{|l} 
Solution \\
\end{tabular} & Scediment & $\begin{array}{c}\text { Oontuct } \\
\text { Trime }\end{array}$ & $\mathrm{pH}$ & $\begin{array}{c}\mathrm{Ca} \\
(\mathrm{ppm})\end{array}$ & $\begin{array}{r}\mathrm{Mg} \\
(\mathrm{ppm}) \\
\end{array}$ & $\begin{array}{r}\mathrm{Na} \\
(\mathrm{pam}) \\
\end{array}$ & $\begin{array}{c}\mathrm{K} \\
(\mathrm{ppm}) \\
\end{array}$ & $\begin{array}{c}\mathrm{si} \\
(\mathrm{ppm})\end{array}$ & $\begin{array}{c}\mathrm{Sr} \\
(\mathrm{prm})\end{array}$ & $\begin{array}{c}\mathrm{C} 1 \\
(\mathrm{ppm})\end{array}$ & $\begin{array}{r}\mathrm{SO}_{4}^{4} \\
(\mathrm{ppmm})\end{array}$ & $\begin{array}{c}\mathrm{NO}_{3} \\
(\mathrm{ppm}) \\
\end{array}$ & $\begin{array}{c}\mathrm{NO}_{2} \\
(\mathrm{ppm})\end{array}$ & $\left.\begin{array}{|l|l}\mathrm{Al} \\
(\mathrm{Apm})\end{array}\right)\left(\begin{array}{c}\mathrm{ClO} \\
(\mathrm{ppmm}\end{array}\right.$ & 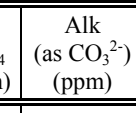 & $\begin{array}{l}\text { ToC } \\
\text { (ppm) }\end{array}$ & \begin{tabular}{|l} 
Cations \\
(meqLL \\
\end{tabular} & $\begin{array}{l}\text { Anions } \\
\text { (mequ/L }\end{array}$ & $\begin{array}{l}\begin{array}{l}\text { Refer- } \\
\text { ence }\end{array} \\
\text { ence }\end{array}$ & $\begin{array}{c}\mathrm{NaNO}_{3} \\
(\mathrm{M}) \\
\end{array}$ & \begin{tabular}{|c}
$\mathrm{NaNO}_{2}$ \\
(M)
\end{tabular} & \begin{tabular}{|c}
$\begin{array}{c}\text { NaOH } \\
(\mathrm{M})\end{array}$ \\
\end{tabular} & $\begin{array}{c}\mathrm{NaAAlO}_{2} \\
(\mathrm{M})\end{array}$ & $\begin{array}{c}\mathrm{N}_{2} \mathrm{a}_{2} \mathrm{CO}_{3} \\
(\mathrm{M})\end{array}$ & $\begin{array}{c}\mathrm{N}_{2} \begin{array}{c}\mathrm{S} \mathrm{SO}_{4} \\
\text { (M) }\end{array} \\
\end{array}$ & $\begin{array}{l}\begin{array}{l}\mathrm{N}_{a} \mathrm{PPO}_{4} \\
(\mathrm{M})\end{array} \\
\end{array}$ & $\begin{array}{l}\mathrm{NaF} \\
(\mathrm{M}) \\
\end{array}$ & \begin{tabular}{|c|c|} 
Nath \\
DDTA \\
(M)
\end{tabular} & \begin{tabular}{|c|}
$\begin{array}{c}\mathrm{Na}_{4} \\
\text { EDDTA } \\
\text { (M) }\end{array}$ \\
\end{tabular} & \begin{tabular}{l|}
$\begin{array}{l}\text { HAA } \\
(\mathrm{M})\end{array}$ \\
\end{tabular} & $\begin{array}{c}\text { Citric A } \\
\text { (M) }\end{array}$ & $\begin{array}{c}\mathrm{CaAN}_{3,3} \\
(\mathrm{M})\end{array}$ & \begin{tabular}{|l|l|l} 
NHANO \\
$($ (M)
\end{tabular} & $\begin{array}{c}\mathrm{KNO}_{3} \\
(\mathrm{M})\end{array}$ & \begin{tabular}{|c|c|}
$\left(\begin{array}{c}\mathrm{NH}_{4}^{4} \mathrm{SO}_{4} \\
(\mathrm{M})\end{array}\right.$ \\
\end{tabular} \\
\hline HGW3 & & & 8.14 & $\begin{array}{l}48.8 \\
\end{array}$ & 114.6 & $\frac{12.1}{32.1}$ & 89.9 & 116.4 & $\begin{array}{l}0.25 \\
.05\end{array}$ & $\frac{127}{127}$ & 175 & $\frac{10.5}{10.5}$ & $<<0.3$ & & 167.5 & 11 & & & & & & & & & & & & & & & & & & & \\
\hline HGW4 & $T$ & & 8.46 & $\mid 67.5$ & $\mid 16.4$ & 227.6 & 13 & 16.2 & 0.28 & $T_{22}$ & \begin{tabular}{|l|l|} 
& 108 \\
\end{tabular} & $\mid 1.7$ & 1 & 1 & $\mid 67.5$ & 0.73 & T & & I & & & & & & & & 1 & & & $T$ & & T & & T & \\
\hline 199-D5.42 & 100-D & 4,14 & $\overline{8.1}$ & 49.5 & 14.6 & 13.2 & 1.7 & 16.5 & & 7.8 & 882.5 & 27.2 & & & 54.1 & & & & (f) & & & & & & & & & & & & & & & & \\
\hline
\end{tabular}




\section{References}

Delegard, C. H. and G. S. Barney. 1983. Effects of Hanford High-Level Waste Components on Sorption of Cobalt, Strontium, Neptunium, Plutonium, and Americium of Hanford Sediments. RHO-RE-ST-1 P, Rockwell Hanford Operations, Richland, Washington.

Gee, G. W. and A. C. Campbell. 1980. Monitoring and Physical Characterization of Unsaturated Zone Transport - Laboratory Analysis. PNL-3304, Pacific Northwest Laboratory, Richland, Washington.

Kaplan, D. I., K. E. Parker, and I. V. Kutynakov. 1998. Radionuclide Distribution Coefficients for Sediments Collected from Borehole 299-E17-21: Final Report for Subtask 1a. PNNL-11966, Pacific Northwest National Laboratory, Richland, Washington.

Serne, R. J. and K. E. Parker. 1999. Estimate of Distribution Coefficients and Leachability of Hexavalent Chromium in 100-D Area Hanford Formation Sediments. BHI-XXX, Bechtel Hanford, Inc., Richland, Washington.

Serne, R. J., J. M. Zachara, and D. S. Burke. 1998. Chemical Information on Tank Supernatants, Cs Adsorption from Tank Liquids onto Hanford Sediments, and Field Observations of Cs Migration from Past Tank Leaks. PNNL-11495, Pacific Northwest National Laboratory, Richland, Washington.

Serne, R. J., J. L. Conca, V. L. LeGore, K. J. Cantrell, C. W. Lindenmeier, J. A. Campbell, J. E. Amonette, and M. I. Wood. 1993. Solid-Waste Leach Characteristics and Contaminant-Sediment Interactions. Volume 1: Batch Leach and Adsorption Tests and Sediment Characterization. PNL-8889, Vol. 1, Pacific Northwest Laboratory, Richland, Washington. 


\section{Appendix B}

Solid Phase (Sediment) Characteristics 
Table B.1. Sediment Characteristics

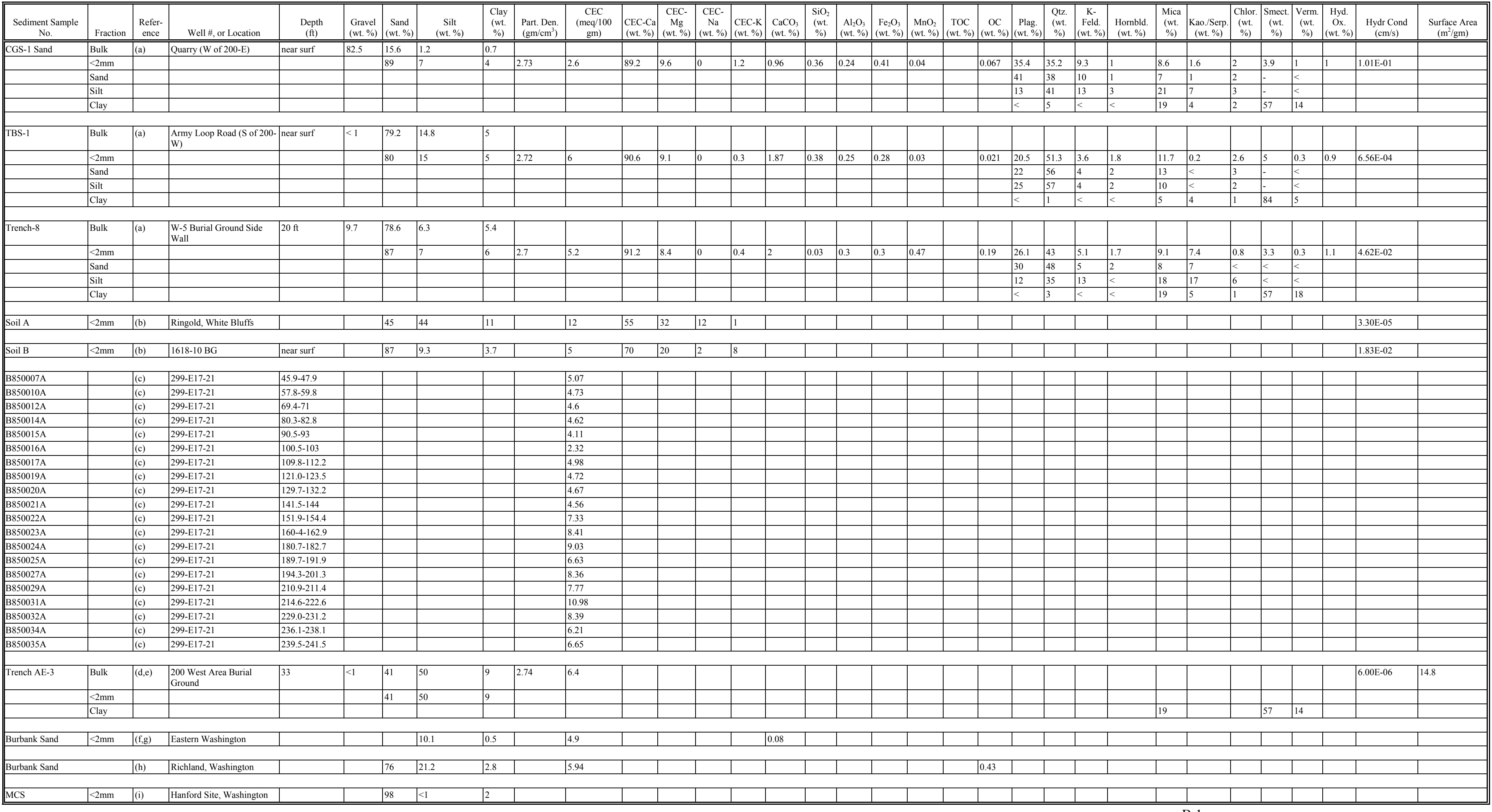




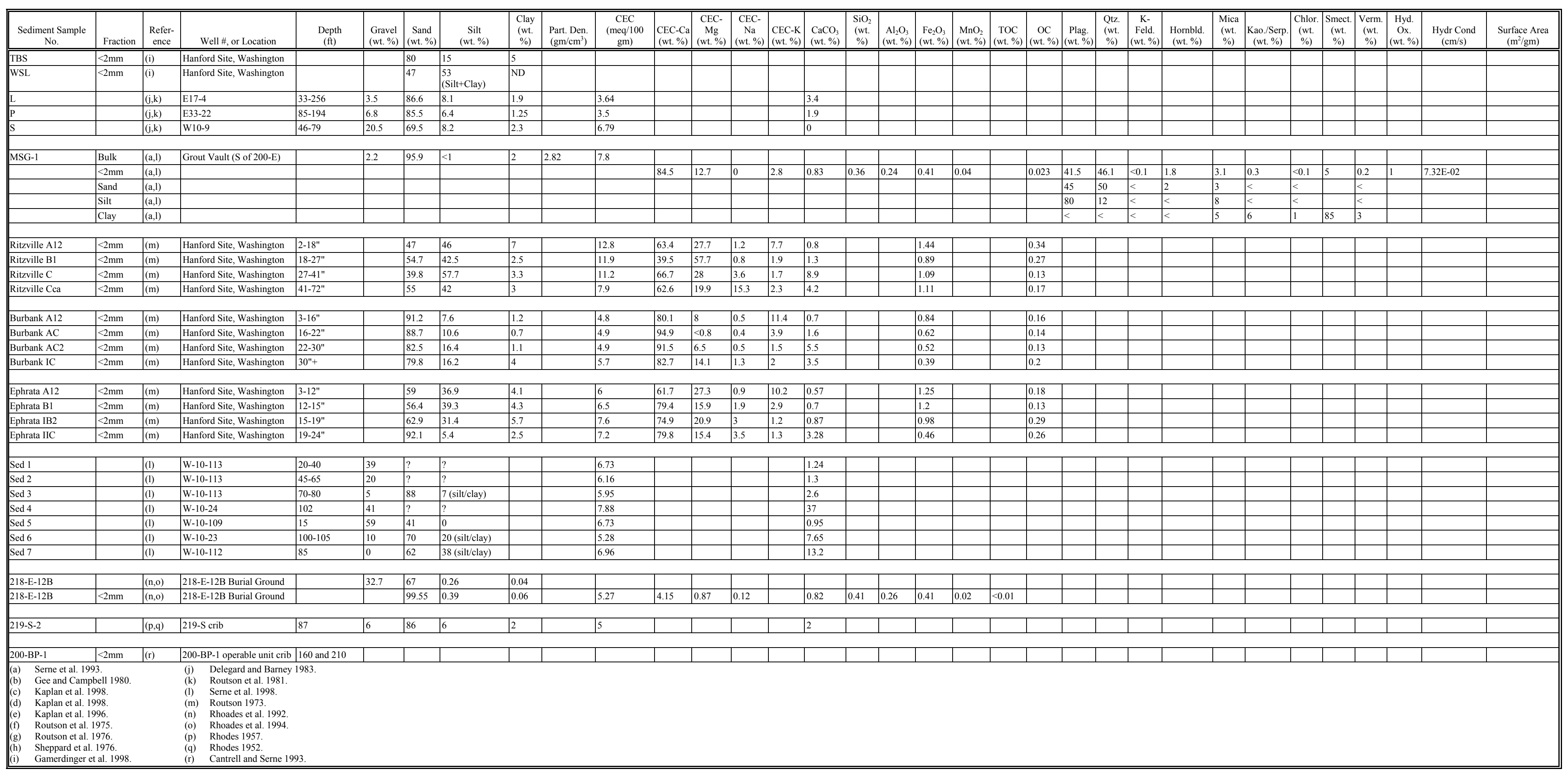




\section{References}

Cantrell, K. J. and R. J. Serne. 1993. Adsorption of ${ }^{60} \mathrm{Co},{ }^{90} \mathrm{Sr},{ }^{99} \mathrm{Tc},{ }^{137} \mathrm{Cs}$, Pu, and Cyanide on 200-BP-1 Sediment. PNL-8612, Pacific Northwest Laboratory, Richland, Washington.

Delegard, C. H. and G. S. Barney. 1983. Effects of Hanford High-Level Waste Components on Sorption of Cobalt, Strontium, Neptunium, Plutonium, and Americium of Hanford Sediments. RHO-RE-ST-1 P, Rockwell Hanford Operations, Richland, Washington.

Gamerdinger, A. P., D. I. Kaplan, and C. T. Resch. 1998. Uranium (VI) Sorption and Transport in Unsaturated, Subsurface Hanford Site Sediments - Effect of Moisture Content and Sediment Texture. PNNL-11975, Pacific Northwest National Laboratory, Richland, Washington.

Gee, G. W. and A. C. Campbell. 1980. Monitoring and Physical Characterization of Unsaturated Zone Transport - Laboratory Analysis. PNL-3304, Pacific Northwest Laboratory, Richland, Washington.

Kaplan, D. I, K. E. Parker, and I. V. Kutynakov. 1998a. Radionuclide Distribution Coefficients for Sediments Collected from Borehole 299-E17-21: Final Report for Subtask 1a. PNNL-11966, Pacific Northwest National Laboratory, Richland, Washington.

Kaplan, D. I., K. E. Parker, and R. D. Orr. 1998. Effects of High-pH and High-Ionic-Strength Groundwater on Iodide, Pertechnetate, and Selenate Sorption to Hanford Sediments: Final Report for Subtask 3a. PNNL-11964, Pacific Northwest National Laboratory, Richland, Washington.

Kaplan, D. I., R. J. Serne, A. T. Owen, J. A. Conca, T. W. Wietsma, and T. L. Gervais. 1996. Radionuclide Adsorption Distribution Coefficients Measured in Hanford Sediments for the Low-Level Waste Performance Assessment Project. PNNL-11485, Pacific Northwest National Laboratory, Richland, Washington.

Rhoades, K., B. N. Bjornstad, R. E. Lewis, S. S. Teel, K. J. Cantrell, R. J. Serne, L. H. Sawyer, J. L. Smoot, J. E. Smoot, J. E. Szecsody, M. S. Wigmosta, and S. K. Wurstner. 1992. Estimation of the Release and Migration of Lead Through Soils and Groundwater at the Hanford Site 218-E-12B Burial Ground. PNL-8356, Pacific Northwest Laboratory, Richland, Washington.

Rhoades, K., B. N. Bjornstad, R. E. Lewis, S. S. Teel, K. J. Cantrell, R. J. Serne, L. H. Sawyer, J. L. Smoot, J. E. Smoot, J. E. Szecsody, M. S. Wigmosta, and S. K. Wurstner. 1994. Estimation of the Release and Migration of Nickel Through Soils and Groundwater at the Hanford Site 218-E-12B Burial Ground. PNL-9791, Pacific Northwest Laboratory, Richland, Washington.

Rhodes, D. W. 1952. Preliminary Studies of Plutonium Adsorption in Hanford Soil. HW-24548, Hanford Works, Richland, Washington.

Rhodes, D. W. 1957. “The Adsorption of Pu by Soil.” Soil Sci. 84:465-471. 
Routson, R. C. 1973. A Review of Studies on Soil-Waste Relationships on the Hanford Reservation from 1944 to 1967. BNWL-1464, Battelle Pacific Northwest Laboratories, Richland, Washington.

Routson, R. C., G. Jansen, and A. V. Robinson. 1975. Sorption of 99Tc, 237Np and 241 Am on Two Subsoils from Differing Weathering Intensity Areas. BNWL-1889, Battelle Pacific Northwest Laboratories, Richland, Washington.

Routson, R. C., G. Jansen, and A. V. Robinson. 1976. "241-Am, 237-Np, and 99-Tc Sorption on Two United States Subsoils from Differing Weathering Intensity Areas.” Health Phys. 33:311-317.

Routson, R. C., G. S. Barney, R. H. Smith, C. H. Delegard, and L. Jensen. 1981. Fission Product Sorption Parameters for Hanford 200-Area Sediment Types. RHO-ST-35, Rockwell Hanford Operations, Richland, Washington.

Serne, R. J., J. L. Conca, V. L. LeGore, K. J. Cantrell, C. W. Lindenmeier, J. A. Campbell, J. E. Amonette, and M. I. Wood. 1993. Solid-Waste Leach Characteristics and Contaminant-Sediment Interactions. Volume 1: Batch Leach and Adsorption Tests and Sediment Characterization. PNL-8889, Vol 1, Pacific Northwest Laboratory, Richland, Washington.

Serne, R. J., J. M. Zachara, and D. S. Burke. 1998. Chemical Information on Tank Supernatants, Cs Adsorption from Tank Liquids onto Hanford Sediments, and Field Observations of Cs Migration from Past Tank Leaks. PNNL-11495, Pacific Northwest National Laboratory, Richland, Washington.

Sheppard, J. C., J. A. Kittrick, and T. L. Hart. 1976. Determination of Distribution Ratios and Diffusion Coefficients of Neptunium, Americium, and Curium in Soil-Aquatic Environments. RLO-2221-T-12-2 (WSU 76/13-33), Washington State University, Pullman, Washington. 


\section{Appendix C}

Carbon Tetrachloride and Acetate $K_{d}$ Values Appropriate for Hanford Soils 


\title{
CARBON TETRACHLORIDE AND ACETATE $K_{d}$ VALUES APPROPRIATE FOR HANFORD SOILS
}

\author{
Kirk Cantre11, 9/23/92
}

\section{Summary}

Based on previous laboratory adsorption data on soils with low organic content, it is expected that adsorption of carbon tetrachloride to Hanford soils (which are low in organic matter) will be very low. For example, Curtis et al. determined the $K_{d}$ value for carbon tetrachloride between Bordon aquifer material $(0.02 \%$ organic carbon) and a solution which simulates the native groundwater from this aquifer to be $0.16 \mathrm{ml} / \mathrm{g}$.

Similarly low values were determined from analytical measurements of carbon tetrachloride measurements made on soils collected from Hanford well 2W11-27 and solutions in contact with these soils. The $K_{d}$ values calculated were $0.005,0.165$ and $0.012 \mathrm{ml} / \mathrm{g}$ for samples collected from depths of 248', $257^{\prime}$ and $262^{\prime}$, respectively.

Adsorption $K_{d}$ values for carbon tetrachloride determined in our laboratories indicate that absorption of carbon tetrachloride onto Hanford soil from Hanford groundwater is essentially zero $\left(K_{d}=0.0 \mathrm{ml} / \mathrm{g}\right)$, within experimental error. This was true for carbon tetrachloride concentrations ranging from 5 to $10,000 \mathrm{ppb}$, both in the presence and absence of $10,000 \mathrm{ppb}$ acetate. Adsorption of acetate was found to be significant at low acetate concentrations. The following $K_{d}$ values were determined, $0.1 \mathrm{ml} / \mathrm{g}$ at $1000 \mathrm{ppm}$ acetate in solution, $0.17 \mathrm{ml} / \mathrm{g}$ at $200 \mathrm{ppm}$ acetate and $3.0 \mathrm{ml} / \mathrm{g}$ at $26 \mathrm{ppm}$ acetate.

Based upon the data provided above, it is suggested that the likely range of $K_{d}$ values appropriate to Hanford conditions for carbon tetrachloride is $0.0 \mathrm{ml} / \mathrm{g}$ to $0.2 \mathrm{ml} / \mathrm{g}$.

Methods

The distribution coefficient or $K_{d}$ is a measure of how well a chemical constituent adsorbs onto a solid material. The $K_{d}$ is defined as the concentration of the constituent of interest in the solid phase divided by its concentration in the liquid phase. The distribution coefficient $\left(\mathrm{K}_{d}\right)$ of $\mathrm{CCl}_{4}$ and acetate between 200 area soil and Hanford groundwater was determined according to the methods outlined in Relyea, Serne and Rai (1980). 
Scintillation counting of ${ }^{14} \mathrm{C}$ labeled compounds was used to determine the concentrations in solution. Non-radioactive compounds were used as carriers to adjust the concentrations of the compound of interest in the initial solutions. The soil was collected from well 299-W11-27 on 11/5/91 at a depth of 200 feet. The Hanford groundwater was collected from well 6-\$3-25.

The $K_{d}$ values were measured by adding approximately one gram of soil to $40 \mathrm{ml}$ of groundwater which was spiked with $\mathrm{CCl}_{4}$. These batch experiments were conducted in glass vials with tefon sealing caps. The concentration of $\mathrm{CCl}_{4}$ on the solid phase was calculated as $[B V-E(V+X)] / W$, where $B$ is the final concentration in the blank, $V$ is the volume of groundwater added, $E$ is the concentration in the sample effluent, $X$ is the excess solution volume left from the pre-equilibration step, and $W$ is the weight of solid aquifer material. Calculation of the $K_{d}$ value was accomplished using equation 1

$$
K_{d}=\frac{B V-E(V+X)}{W E}
$$

\section{$\underline{\text { Results }}$}

Results of our laboratory experiments are collected in Table 1 . Most of the $K_{d}$ values determined for $\mathrm{CCl}_{4}$ are negative. Negative $\mathrm{K}_{d}$ values are not physically possible. Negative values resulted because the blanks actually lost slightly more $\mathrm{CCl}_{4}$ than the vials which contained soil. From equation 1 , we can see that this will result in a negative value for $K_{d}$. The vials used in these experiments were composed of glass with a teflon sealing cap. Apparently $\mathrm{CCl}_{4}$ has an affinity for teflon because it should not absorb to any significant amount onto glass. The average value of $\mathrm{K}_{d}$ for $\mathrm{CCl}_{4}$ determined from all the experiments was -0.8. Using the methods in Data Analys is for Scientists and Engineers (Meyer S. L., 1975), the average standard deviation of the $K_{d}$ values was estimated to be 1.3. Based on these data it appears that the $\mathrm{K}_{d}$ value for $\mathrm{CCl}_{4}$ on Hanford soil is $0.0 \mathrm{~mL} / \mathrm{g}$, with an upper bound value of approximately $1.3 \mathrm{~mL} / \mathrm{g}$. 
Table 1. $K_{d}$ values determined for carbon tetrachloride, both in the presence and absence of acetate, and $k_{d}$ values for acetate.

$\begin{array}{rcc}\mathrm{CC1}_{4} \text { Concentration }(\mathrm{ppb}) & \text { Acetate Concentration }(\mathrm{ppm}) & \mathrm{K}_{\mathrm{d}}\left(\mathrm{CCl}_{4}\right)(\mathrm{mL} / \mathrm{g}) \\ 10,000 & 0 & -1.8 \\ 5,000 & 0 & -0.2 \\ 500 & 0 & -1.5 \\ 50 & 0 & -1.6 \\ 5 & 0 & -0.5 \\ 10,000 & 10 & 0.0 \\ 5,000 & 10 & 0.3 \\ 500 & 10 & -1.5 \\ 50 & 10 & -0.6 \\ 5 & 10 & -0.6 \\ & & 0.1 \\ 0 & 1,000 & 0.2 \\ 0 & 200 & 3.1 \\ 0 & 26 & \mathrm{~K}_{\mathrm{d}}(\mathrm{acetate})(\mathrm{mL} / \mathrm{g}) \\ & & 0.1\end{array}$




\section{C.1 References}

Meyer, S. L. 1975. Data Analysis for Scientists and Engineers. John Wiley \& Sons, New York.

Relyea, J. F., R. J. Serne, and D. Rai. 1980. Methods for Determining Radionuclide Retardation Factors: Status Report. PNL-3349, Pacific Northwest Laboratory, Richland, Washington. 


\section{Distribution}

No. of

\section{Copies}

\section{OFFSITE}

M. P. Connelly

HydroGeologic, Inc.

3250 Port of Benton Boulevard

Richland, WA 99352

P. Vaughn

Sandia National Laboratories

P.O. Box 5800

Albuquerque, NM 87185

ONSITE

2 DOE Office of River Protection

C. A. Babel

R. M. Yasek

8 DOE Richland Operations Office

B. L. Foley

J. P. Hanson

R. D. Hildebrand

K. A. Kapsi

J. G. Morse

K. M. Thompson

DOE Public Reading Room (2)

8 Bechtel Hanford, Inc.

L. R. Curry

H0-09

A. G. Dada

K. R. Fecht

B. H. Ford

M. N. Jarayssi

G. B. Mitchem

M. R. Schwab

R. L. Vaughan

H6-60

H6-60

K8-50

A5-13

K8-50

A5-13

A5-13

$\mathrm{H} 2-53$
No. of

Copies

6 CH2M HILL Hanford, Inc.

M.Y.L. Mandis H9-01

W. J. McMahon H9-03

V. J. Rohay H0-19

K. M. Singleton H9-02

L. C. Swanson H9-02

C. D. Wittreich H9-03

4 CH2M HILL Hanford Group, Inc.
A. J. Knepp
$\mathrm{H} 0-22$
T. E. Jones
L7-12
F. M. Mann
$\mathrm{H} 0-22$
D. A. Myers
$\mathrm{H} 0-22$

Fluor Federal Services

R. Khaleel

K6-17

Fluor Hanford, Inc.

M. I. Wood

H8-44

48 Pacific Northwest National Laboratory

D. H. Bacon K9-33

C. F. Brown P8-37

R. W. Bryce K6-75

J. G. Bush K6-96

A. L. Bunn K6-85

K. J. Cantrell (5) K6-81

J. L. Devary K6-96

R. L. Dirkes K6-75

P. W. Eslinger K6-80

M. J. Fayer K9-33

E. J. Freeman K9-36

M. D. Freshley H0-21 
PNNL-13895

No. of

Copies

J. S. Fruchter

G. W. Gee

G. R. Guensch

D. G. Horton

C. T. Kincaid

K. M. Krupka

G. V. Last (5)

W. J. Martin

C. J. Murray

W. E. Nichols

T. L. Page

W. A. Perkins
No. of

Copies

K6-96

K9-33

K9-33

K6-81

K9-33

K6-81

K6-81

K6-81

K6-81

K9-33

K9-18

K9-36
S. P. Reidel

M. C. Richmond

R. G. Riley

R. J. Serne (5)

R. M. Smith

P. D. Thorne

W. Um

A. L. Ward

S. B. Yabusaki

J. M. Zachara

Hanford Technical Library (2)
K6-81

K9-33

K6-96

P8-37

K6-96

K9-33

P8-37

K9-33

K9-36

K8-96

P8-55

Distr.2 